\title{
Visible-Light-Induced Amination of Quinoline at the C8 Position via Post-Coordinated Interligand-Coupling Strategy under Mild Conditions
}

\author{
He-Long Peng, ${ }^{\dagger}$ Yinwu Li, ${ }^{\dagger}$ Xing-Yang Chen, ${ }^{\dagger}$ Li-Ping Li, ${ }^{\dagger}$ Zhuofeng Ke, ${ }^{*},{ }^{\star}$ and Bao-Hui Ye ${ }^{*, \dagger}$ \\ † MOE Key Laboratory of Bioinorganic and Synthetic Chemistry, School of Chemistry, Sun Yat-sen University, \\ Guangzhou 510275, Guangdong, China. E-mail: cesybh@mail.sysu.edu.cn (B.-H. Y.) \\ * School of Materials Science \& Engineering, Sun Yat-sen University, Guangzhou 510275, Guangdong, China. E- \\ mail: kezhf3@mail.sysu.edu.cn (Z. K.)
}

\section{Table of Contents}

S1. Materials and Methods S2

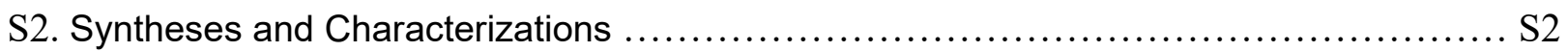

S2.1 Synthesis of 7-Chloro-2-phenylquinoline (Cpq) and 7-Bromo-2-phenylquinoline (Bpq) ...............S2

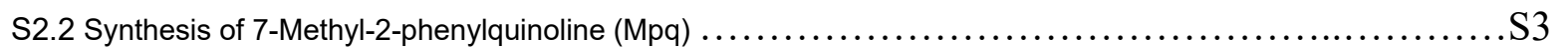

S2.3 General Procedure for the Synthesis of Enantiopure $\operatorname{Ir}(\mathrm{III})$ Amino Acid Complexes....................S3

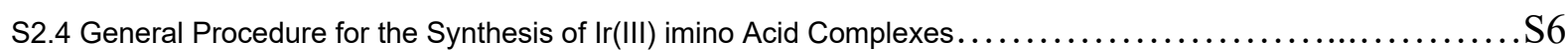

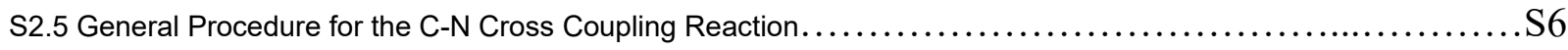

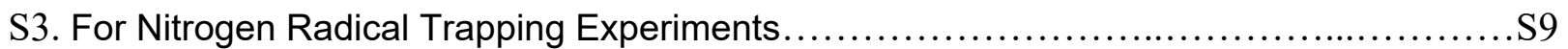

S4. For Carbon Radical Trapping Experiments ....................................... 9

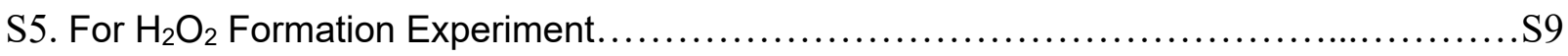

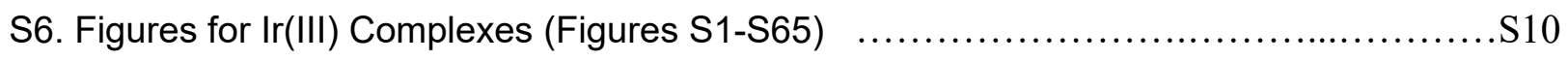

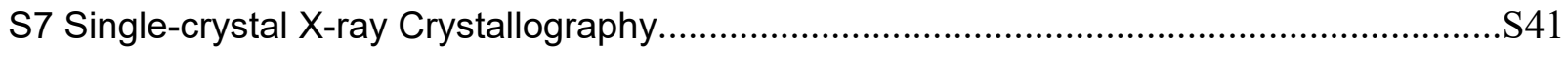

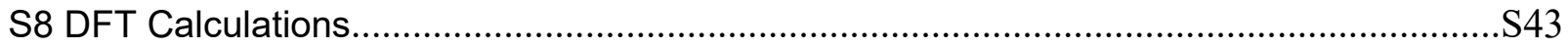

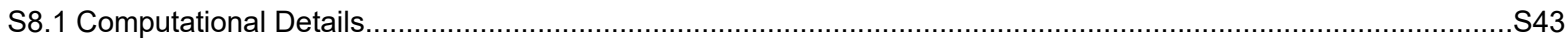

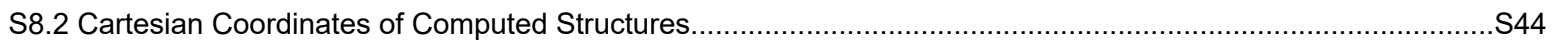

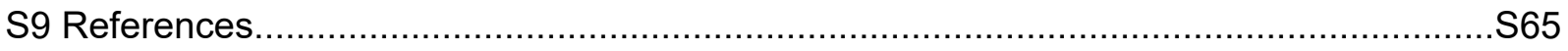




\section{S1. Materials and Methods}

All chemicals were commercially available and used without further purification unless otherwise noted. 7-Chloro-2-phenylquinoline $(\mathrm{Cpq})$ and 7-bromo-2-phenylquinoline $(\mathrm{Bpq})$ were synthesized according to the literature. ${ }^{[1]}$ The enantiopure $\Lambda$-[ $\operatorname{lr}(\mathrm{pq})_{2}\left(\mathrm{MeCN}_{2}\right]\left(\mathrm{PF}_{6}\right)$, $\Delta-\left[\operatorname{Ir}(\mathrm{pq})_{2}(\mathrm{MeCN})_{2}\right]\left(\mathrm{PF}_{6}\right), \Lambda-\left[\operatorname{Ir}(\mathrm{Mpq})_{2}(\mathrm{MeCN})_{2}\right]\left(\mathrm{PF}_{6}\right), \Lambda-\left[\operatorname{Ir}(\mathrm{Cpq})_{2}(\mathrm{MeCN})_{2}\right]\left(\mathrm{PF}_{6}\right)$, and $\Lambda-\left[\operatorname{Ir}(\mathrm{Bpq})_{2}(\mathrm{MeCN})_{2}\right]\left(\mathrm{PF}_{6}\right)(\mathrm{ee} \geq 99 \%)(\mathrm{pq}$ is $2-$ phenylquinoline, Mpq is 7-methyl-2-phenylquinoline, $\mathrm{Cpq}$ is 7-chloro-2-phenylquinoline, and Bpq is 7-bromo-2-phenylquinoline) were synthesized according to the literatures. ${ }^{[2]}$ Elemental $(\mathrm{C}, \mathrm{H}$ and $\mathrm{N})$ analyses were carried out on an Elementar Vario EL analyzer. Electrospray ionization mass spectra (ESI-MS) were obtained on a Thermo LTQ XL mass spectrometer. High-resolution mass spectrum (HRMS) was recorded on a Thermo MAT95XP-HRMS analyzer in an ESI mode. ${ }^{1} \mathrm{H}$ and ${ }^{13} \mathrm{C}$ NMR spectra were recorded in $\mathrm{CDCl}_{3}$ on a Bruker AV 400 spectrometer at room temperature by using the solvent as the internal standard. Electronic absorption spectra were obtained on a Persee TU-1901 UV-vis spectrophotometer. Electrochemical measurements were carried out on a $\mathrm{CHI}-730 \mathrm{C}$ electrochemistry system in $\mathrm{CH}_{3} \mathrm{CN}$. A three-electrode assembly comprising a glassy-carbon working electrode, a Pt auxiliary electrode, and a Pt-wire reference electrode were used. All experiments were carried out at room temperature using $0.1 \mathrm{~mol} / \mathrm{L} n-\mathrm{Bu}_{4} \mathrm{NPF} 6$ as the supporting electrolyte. The reference electrode was assumed to be stable and was referenced externally to ferrocene (0.39 V). CD spectra were measured on a JASCO J-810 CD spectropolarimeter. Emission spectra and lifetimes were determined on an FLS920 fluorescence lifetime and steady-state spectrometer at room temperature. Quantum yields of air-equilibrated MeOH solution were determined on a FLSP920 fluorescence spectrophotometer with $\mathrm{Ru}(\mathrm{bpy}){ }_{3} \mathrm{Cl}_{2}$ in air-equilibrated water solution $(\Phi=0.028)$ as a standard.

\section{S2. Syntheses and Characterizations}

\section{S2.1 Synthesis of 7-Chloro-2-phenylquinoline (Cpq) and 7-Bromo-2-phenylquinoline (Bpq).}

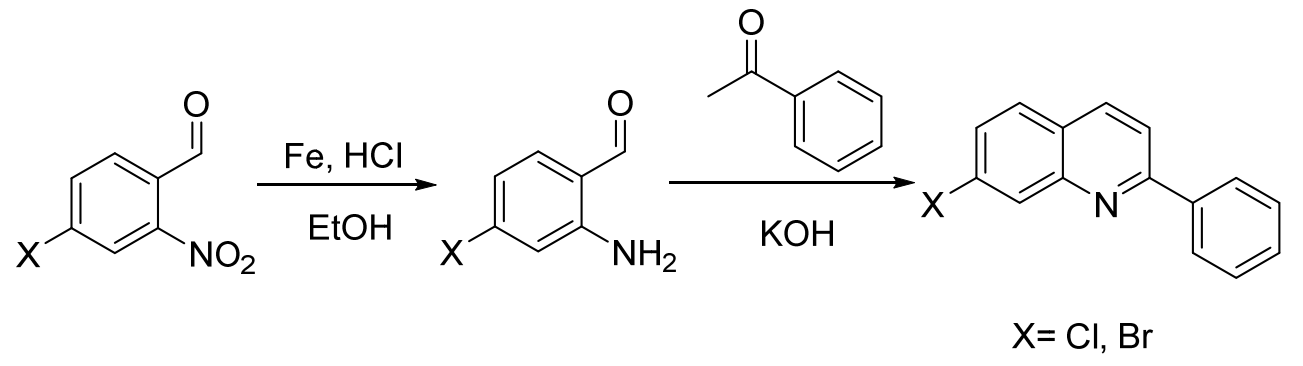

20.0 mmol of 4-chloro-2-nitrobenzaldehyde or 4-bromo-2-nitrobenzaldehyde was reduced by 80.0 mmol iron powder in the presence of $83.0 \mu \mathrm{L}$ concentrated $\mathrm{HCl}$ in refluxing $\mathrm{EtOH}$. The reaction was monitored by thin-layer chromatography (TLC) and usually completed within 30-40 minutes. After that, 20.0 mmol acetophenone and 24.0 mmol KOH were added. The mixture was then stirred at reflux for a further 40-60 minutes. When the reaction completed, the excess iron power was filtered and the solvent was removed under reduced pressure. The crude product was purified by recrystallization with a mixture of $n$-hexane $/ \mathrm{CH}_{2} \mathrm{Cl}_{2}(2 / 1 \mathrm{v} / \mathrm{v})$ to get the target compounds.

For Cpq. Yield, 4.25 g, 89\%. Anal. Calcd. for $\mathrm{C}_{15} \mathrm{H}_{10} \mathrm{ClN}$ : C 75.16, H 4.21, N 5.84; found: C 75.32, H 4.42, N 5.61\%. ESIMS: $\mathrm{m} / \mathrm{z}=239.05[\mathrm{M}+\mathrm{H}]^{+} .{ }^{1} \mathrm{H}$ NMR $\left(400 \mathrm{MHz}, \mathrm{CDCl}_{3}\right) \delta 8.23-8.13(\mathrm{~m}, 4 \mathrm{H}), 7.88(\mathrm{~d}, \mathrm{~J}=8.6 \mathrm{~Hz}, 1 \mathrm{H}), 7.76(\mathrm{~d}, \mathrm{~J}=8.7$ 
$\mathrm{Hz}, 1 \mathrm{H}), 7.51$ (ddd, J = 22.1, 8.1, 4.5 Hz, 4H). ${ }^{13} \mathrm{C}$ NMR $\left(101 \mathrm{MHz}, \mathrm{CDCl}_{3}\right) \delta$ 158.39, 148.81, 139.32, 136.69, 135.61, 129.80, $129.04,129.04,128.86,128.79,127.72,127.72,127.42,125.66,119.25$.

For Bpq. Yield, 5.15 g, 91\%. Anal. Calcd. for $\mathrm{C}_{15} \mathrm{H}_{10} \mathrm{BrN}$ : C 63.40, H 3.55, N 4.93; found: C 63.73, H 3.73, N 4.62\%. ESIMS: $\mathrm{m} / \mathrm{z}=284.22[\mathrm{M}+\mathrm{H}]^{+} .{ }^{1} \mathrm{H}$ NMR $\left(400 \mathrm{MHz}, \mathrm{CDCl}_{3}\right) \delta 8.37(\mathrm{~d}, \mathrm{~J}=1.9 \mathrm{~Hz}, 1 \mathrm{H}), 8.22-8.13(\mathrm{~m}, 3 \mathrm{H}), 7.90(\mathrm{~d}, \mathrm{~J}=8.6$ $\mathrm{Hz}, 1 \mathrm{H}), 7.70(\mathrm{~d}, \mathrm{~J}=8.6 \mathrm{~Hz}, 1 \mathrm{H}), 7.61(\mathrm{dd}, \mathrm{J}=8.6,1.9 \mathrm{~Hz}, 1 \mathrm{H}), 7.57-7.45(\mathrm{~m}, 3 \mathrm{H}) .{ }^{13 \mathrm{C}} \mathrm{NMR}\left(101 \mathrm{MHz}, \mathrm{CDCl}_{3}\right) \delta 158.34$, 149.06, 139.31, 136.79, 132.22, 129.92, 129.83, 129.05, 129.05, 128.85, 127.73, 127.73, 125.92, 123.87, 119.40.

S2.2 Synthesis of 7-Methyl-2-phenylquinoline (Mpq).<smiles>Brc1ccc2ccc(-c3ccccc3)nc2c1</smiles>

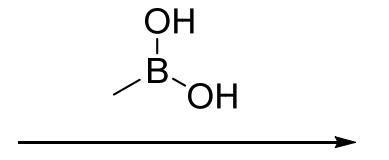

Pd Cat.<smiles>Cc1ccc2ccc(-c3ccccc3)nc2c1</smiles>

7-Bromo-2-phenylquinoline (4.26 g, 15.0 mmol), methyl boric acid (30.0 mmol), cesium carbonate (30.0 mmol) and triphenylphosphine palladium ( 2 mol\%) were added into a $100 \mathrm{~mL}$ round-bottomed flask with $50 \mathrm{~mL}$ 1,4-dioxane as the solvent. The mixture was stirred at reflux for 3-4 days (monitored by TLC). After that, the catalyst was removed by filtration and the solvent was removed under reduced pressure. Then the solid was dissolved in 6o mL DCM and washed by water $(3 \times 30 \mathrm{~mL})$. The organic phase was dried by anhydrous $\mathrm{Na}_{2} \mathrm{SO}_{4}$, then the solvent was removed and the crude product was purified by a silica gel column (300-400 mesh) with a n-hexane/ethyl acetate (100/1 to 100/10 v/v) as an eluent to get Mpq. Yield, $1.91 \mathrm{~g}, 58 \%$. Anal. Calcd. for $\mathrm{C}_{16} \mathrm{H}_{13} \mathrm{~N}$ : C 87.64, H 5.98, N 6.39; Found: C 87.42, H 5.77, 6.21\%. ESI-MS: $\mathrm{m} / \mathrm{z}=220.31[\mathrm{M}+\mathrm{H}]^{+} .{ }^{1} \mathrm{H}$ NMR $\left(400 \mathrm{MHz}, \mathrm{CDCl}_{3}\right) \delta 8.18(\mathrm{~d}, \mathrm{~J}=7.8 \mathrm{~Hz}, 2 \mathrm{H}), 8.13(\mathrm{~d}, \mathrm{~J}=8.5 \mathrm{~Hz}, 1 \mathrm{H}), 8.00(\mathrm{~s}$, 1H), $7.79(\mathrm{~d}, \mathrm{~J}=8.5 \mathrm{~Hz}, 1 \mathrm{H}), 7.70(\mathrm{~d}, \mathrm{~J}=8.2 \mathrm{~Hz}, 1 \mathrm{H}), 7.54(\mathrm{t}, \mathrm{J}=7.5 \mathrm{~Hz}, 2 \mathrm{H}), 7.50-7.45(\mathrm{~m}, 1 \mathrm{H}), 7.36(\mathrm{~d}, \mathrm{~J}=8.2 \mathrm{~Hz}$, 1H), 2.59 (s, $3 \mathrm{H}) .{ }_{13 \mathrm{C}} \mathrm{NMR}\left(101 \mathrm{MHz}, \mathrm{CDCl}_{3}\right.$ ) $\delta$ 157.37, 148.62, 139.98, 139.94, 136.50, 129.29, 128.88, 128.88, 128.88, $128.62,127.62,127.62,127.18,125.33,118.26,21.99$.

\section{S2.3 General Procedure for the Synthesis of Enantiopure Ir(III) Amino Acid Complexes.}

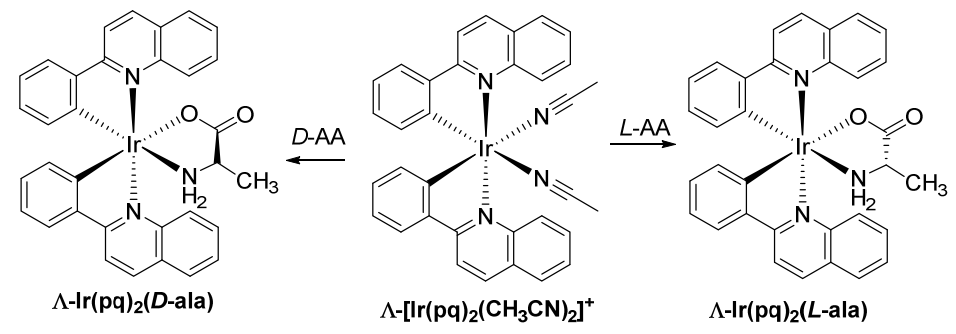

For $\Lambda$-[Ir(pq) $)_{2}(\mathbf{L}-$ ala) $]$ or $\Delta$-[Ir(pq) 2 (D-ala)]. Yield, $674 \mathrm{mg}, 98 \%$. Anal. calcd for $\mathrm{C}_{33} \mathrm{H}_{26} \operatorname{IrN}_{3} \mathrm{O}_{2}: \mathrm{C}_{57.54}, \mathrm{H} 3.80$, N 6.10; found: C 57.75, H 3.68, N 6.32\%. ESI-MS: $\mathrm{m} / \mathrm{z}=690.11[\mathrm{M}+\mathrm{H}]^{+} .{ }^{1} \mathrm{H}$ NMR (400 MHz, $\left.\mathrm{CDCl}_{3}\right): \delta 8.81(\mathrm{~d}, \mathrm{~J}=8.8$ $\mathrm{Hz}, 1 \mathrm{H}), 8.21(\mathrm{~d}, \mathrm{~J}=8.8 \mathrm{~Hz}, 1 \mathrm{H}), 8.16(\mathrm{~d}, \mathrm{~J}=8.8 \mathrm{~Hz}, 1 \mathrm{H}), 8.07(\mathrm{~d}, \mathrm{~J}=8.8 \mathrm{~Hz}, 1 \mathrm{H}), 8.00(\mathrm{t}, 2 \mathrm{H}), 7.83(\mathrm{dd}, \mathrm{J}=11.7,8.5 \mathrm{~Hz}$, $3 \mathrm{H}), 7.71(\mathrm{~d}, \mathrm{~J}=7.8 \mathrm{~Hz}, 1 \mathrm{H}), 7.61-7.54(\mathrm{~m}, 1 \mathrm{H}), 7.53-7.45(\mathrm{~m}, 5 \mathrm{H}), 6.95(\mathrm{t}, 1 \mathrm{H}), 6.89(\mathrm{t}, 2 \mathrm{H}), 6.66(\mathrm{t}, 1 \mathrm{H}), 6.57(\mathrm{t}, 1 \mathrm{H})$, 
$6.26(\mathrm{~d}, \mathrm{~J}=7.6 \mathrm{~Hz}, 1 \mathrm{H}), 3.53(\mathrm{dd}, \mathrm{J}=11.6,6.5 \mathrm{~Hz}, 1 \mathrm{H}), 2.96(\mathrm{dt}, 1 \mathrm{H}), 2.12(\mathrm{t}, 1 \mathrm{H}), 0.81(\mathrm{~d}, \mathrm{~J}=7.0 \mathrm{~Hz}, 3 \mathrm{H}) .{ }^{13 \mathrm{C}} \mathrm{NMR}(101$ $\left.\mathrm{MHz}, \mathrm{CDCl}_{3}\right): \delta 180.65,171.82,170.62,151.29,150.13,148.99,148.44,146.55,146.07,139.02,138.45,136.98,134.77$, $132.24,131.11,130.01,129.85,129.81$, 129.50, 127.93, 127.83, 127.71, 127.00, 126.37, 126.22, 125.99, 123.93, 121.92, 120.99, 117.77, 116.54, 51.48, 22.01. CD $\left(\Delta \varepsilon / \mathrm{M}^{-1} \mathrm{~cm}^{-1}, \mathrm{MeOH}\right)$ for $\Lambda$-[Ir(pq) (L-ala)]: 270(-26), 309(+52), 354(+54), 470(-8). $\mathrm{CD}\left(\Delta \varepsilon / \mathrm{M}^{-1} \mathrm{~cm}^{-1}, \mathrm{MeOH}\right)$ for $\Delta-\left[\mathbf{I r}(\mathbf{p q})_{2}\right.$ (D-ala)]: 270(+29), 309(-56), 354(-54), 470(+8).

For $\Lambda$-[Ir(pq) $)_{2}$ (D-ala)] or $\Delta$-[Ir(pq) 2 (L-ala)]. Yield, $668 \mathrm{mg}, 97 \%$. Anal. calcd for $\mathrm{C}_{33} \mathrm{H}_{26} \mathrm{IrN}_{3} \mathrm{O}_{2}: \mathrm{C}$ 57.54, $\mathrm{H} 3.80$, N 6.10; found: C 57.48, H 3.54, N 6.03\%. ESI-MS: $\mathrm{m} / \mathrm{z}=690.22[\mathrm{M}+\mathrm{H}]^{+} .{ }^{1} \mathrm{H}$ NMR (40o MHz, $\left.\mathrm{CDCl}_{3}\right)$ : 88.52 (d, J = 8.5 $\mathrm{Hz}, 1 \mathrm{H}), 8.23(\mathrm{~d}, \mathrm{~J}=8.7 \mathrm{~Hz}, 1 \mathrm{H}), 8.20(\mathrm{~d}, \mathrm{~J}=8.8 \mathrm{~Hz}, 1 \mathrm{H}), 8.09(\mathrm{~d}, \mathrm{~J}=7.2 \mathrm{~Hz}, 1 \mathrm{H}), 8.06(\mathrm{~d}, \mathrm{~J}=7.2 \mathrm{~Hz}, 1 \mathrm{H}), 7.94-7.88$ (m, 1H), $7.85(\mathrm{~d}, \mathrm{~J}=7.3 \mathrm{~Hz}, 1 \mathrm{H}), 7.82-7.78(\mathrm{~m}, 3 \mathrm{H}), 7.55-7.47(\mathrm{~m}, 4 \mathrm{H}), 7.02-6.91(\mathrm{~m}, 3 \mathrm{H}), 6.74(\mathrm{t}, 1 \mathrm{H}), 6.61(\mathrm{t}, 1 \mathrm{H})$, $6.32(\mathrm{~d}, \mathrm{~J}=7.1 \mathrm{~Hz}, 1 \mathrm{H}), 3.29(\mathrm{t}, 1 \mathrm{H}), 2.71(\mathrm{dd}, \mathrm{J}=10.8,6.3 \mathrm{~Hz}, 1 \mathrm{H}), 2.44-2.29(\mathrm{~m}, 1 \mathrm{H}), 0.91(\mathrm{~d}, \mathrm{~J}=6.9 \mathrm{~Hz}, 3 \mathrm{H}) .{ }^{13} \mathrm{C}$ NMR (101 MHz, $\left.\mathrm{CDCl}_{3}\right): \delta$ 180.41, 171.84, 170.27, 151.80, 150.98, 149.31, 147.66, 146.58, 145.93, 138.72, 138.34, 137.49, $135.15,132.24,131.04,130.39,129.76,129.45,128.15,127.98,127.13,127.01,126.75,126.48,126.23,125.83,124.07$, 121.96, 121.05, 117.79, 116.53, 54.14, 22.45. $\mathrm{CD}\left(\Delta \varepsilon / \mathrm{M}^{-1} \mathrm{~cm}^{-1}, \mathrm{MeOH}\right)$ for $\Lambda$-[Ir(pq) $\left.\mathbf{2}(\mathbf{D}-\mathbf{a l a})\right]: 273(-25), 308(+56)$, 354(+41), 467(-10). $\mathrm{CD}\left(\Delta \varepsilon / \mathrm{M}^{-1} \mathrm{~cm}^{-1}, \mathrm{MeOH}\right)$ for $\Delta$-[Ir(pq) $\left.\mathbf{2}(\mathbf{L}-\mathbf{a l a})\right]:$ 272(+31), 307(-66), 354(-48), 468(+12).

For $\Delta-\left[\operatorname{Ir}(\mathbf{p q})_{2}\right.$ (D-val)] (val is valine). Yield, $730 \mathrm{mg}, 98 \%$. Anal. calcd for $\mathrm{C}_{35} \mathrm{H}_{30} \mathrm{IrN}_{3} \mathrm{O}_{2}$ : $\mathrm{C} 58.64, \mathrm{H} 4.22, \mathrm{~N} 5.86$; found: $\mathrm{C} 58.33, \mathrm{H} 4.01 \mathrm{~N} 5.66 \%$. ESI-MS: $\mathrm{m} / \mathrm{z}=718.22[\mathrm{M}+\mathrm{H}]^{+} .{ }^{1} \mathrm{H}$ NMR $\left(400 \mathrm{MHz}, \mathrm{CDCl}_{3}\right): 88.80(\mathrm{~d}, \mathrm{~J}=8.9 \mathrm{~Hz}$, $1 \mathrm{H}), 8.28(\mathrm{~d}, \mathrm{~J}=8.7 \mathrm{~Hz}, 1 \mathrm{H}), 8.16(\mathrm{dd}, \mathrm{J}=12.2,8.8 \mathrm{~Hz}, 2 \mathrm{H}), 8.05(\mathrm{~d}, \mathrm{~J}=8.8 \mathrm{~Hz}, 1 \mathrm{H}), 7.98(\mathrm{~d}, \mathrm{~J}=8.3 \mathrm{~Hz}, 1 \mathrm{H}), 7.89(\mathrm{~d}, \mathrm{~J}$ $=8.0 \mathrm{~Hz}, 1 \mathrm{H}), 7.86(\mathrm{~d}, \mathrm{~J}=7.4 \mathrm{~Hz}, 1 \mathrm{H}), 7.82(\mathrm{~d}, \mathrm{~J}=7.8 \mathrm{~Hz}, 1 \mathrm{H}), 7.76(\mathrm{~d}, \mathrm{~J}=7.9 \mathrm{~Hz}, 1 \mathrm{H}), 7.59(\mathrm{t}, 1 \mathrm{H}), 7.49(\mathrm{dt}, 3 \mathrm{H}), 7.02$ $(\mathrm{t}, 1 \mathrm{H}), 6.96-6.90(\mathrm{~m}, 2 \mathrm{H}), 6.75(\mathrm{t}, 1 \mathrm{H}), 6.62(\mathrm{t}, 1 \mathrm{H}), 6.35(\mathrm{~d}, \mathrm{~J}=7.7 \mathrm{~Hz}, 1 \mathrm{H}), 2.95(\mathrm{dd}, \mathrm{J}=10.9,6.8 \mathrm{~Hz}, 1 \mathrm{H}), 2.74(\mathrm{~d}, \mathrm{~J}=$ $7.0 \mathrm{~Hz}, 1 \mathrm{H}), 2.26(\mathrm{t}, 2 \mathrm{H}), 0.60(\mathrm{~d}, \mathrm{~J}=7.1 \mathrm{~Hz}, 3 \mathrm{H}),-0.07(\mathrm{~d}, \mathrm{~J}=6.8 \mathrm{~Hz}, 3 \mathrm{H}) .{ }^{13} \mathrm{C}$ NMR $\left(101 \mathrm{MHz}, \mathrm{CDCl}_{3}\right): \delta$ 179.53, 171.83 , 170.55, 151.28, 150.32, 148.87, 148.60, 146.43, 146.08, 139.01, 138.47, 137.13, 134.94, 131.98, 130.85, 130.04, 129.88, 129.69, 128.39, 127.94, 127.83, 127.79, 127.06, 126.45, 126.28, 126.02, 123.91, 121.94, 121.04, 117.83, 116.58, 60.51, 30.15, 19.63, 14.71. $\mathrm{CD}\left(\Delta \varepsilon / \mathrm{M}^{-1} \mathrm{~cm}^{-1}, \mathrm{MeOH}\right): 271(+22), 309(-46), 354(-50), 478(+8)$.

For $\Delta$-[Ir(pq) $\left(\mathbf{D}\right.$-ser)] (ser is serine). Yield, $676 \mathrm{mg}, 98 \%$. Anal. calcd for $\mathrm{C}_{33} \mathrm{H}_{24} \mathrm{IrN}_{3} \mathrm{O}_{3}: \mathrm{C}_{56.24}, \mathrm{H} 3.72, \mathrm{~N}$ 5.96; found: $\mathrm{C} 56.66, \mathrm{H} 3.53, \mathrm{~N} 6.04 \%$. ESI-MS: $\mathrm{m} / \mathrm{z}=706.18[\mathrm{M}+\mathrm{H}]^{+} .{ }^{1} \mathrm{H}$ NMR $\left(400 \mathrm{MHz}, \mathrm{CDCl}_{3}\right): 88.72(\mathrm{~d}, \mathrm{~J}=8.8 \mathrm{~Hz}$, $1 \mathrm{H}), 8.27(\mathrm{~d}, \mathrm{~J}=8.7 \mathrm{~Hz}, 1 \mathrm{H}), 8.19(\mathrm{~d}, \mathrm{~J}=8.7 \mathrm{~Hz}, 1 \mathrm{H}), 8.11(\mathrm{~d}, \mathrm{~J}=8.8 \mathrm{~Hz}, 1 \mathrm{H}), 8.04(\mathrm{t}, 2 \mathrm{H}), 7.85(\mathrm{q}, 3 \mathrm{H}), 7.76(\mathrm{~d}, \mathrm{~J}=7.9$ $\mathrm{Hz}, 1 \mathrm{H}), 7.64-7.47(\mathrm{~m}, 4 \mathrm{H}), 6.99(\mathrm{t}, 1 \mathrm{H}), 6.96-6.85(\mathrm{~m}, 2 \mathrm{H}), 6.72(\mathrm{t}, 1 \mathrm{H}), 6.61(\mathrm{t}, 1 \mathrm{H}), 6.30(\mathrm{~d}, \mathrm{~J}=7.7 \mathrm{~Hz}, 1 \mathrm{H}), 3.71(\mathrm{~d}, \mathrm{~J}=$ $11.3 \mathrm{~Hz}, 1 \mathrm{H}), 3.18-2.88(\mathrm{~m}, 4 \mathrm{H}), 2.20(\mathrm{t}, \mathrm{J}=7.1 \mathrm{~Hz}, 1 \mathrm{H}) .{ }^{13} \mathrm{C} \mathrm{NMR}\left(101 \mathrm{MHz}, \mathrm{CDCl}_{3}\right.$ ): $\delta$ 178.91, 170.47, 148.93, 148.53, $146.47,146.14,139.28,138.56,136.92,134.98$, 131.90, 131.30, 129.91, 129.87, 129.72, 128.10, 127.99, 127.75, 126.91, 126.45, 126.22, 126.12, 123.87, 122.07, 121.16, 117.79, 116.57, 64.83, 56.84. CD ( $\left.\Delta \varepsilon / \mathrm{M}^{-1} \mathrm{~cm}^{-1}, \mathrm{MeOH}\right): 271(+28), 309(-50)$, $354(-53), 483(+8)$. 
For $\Delta$-[Ir(pq) $)_{2}$ (D-pro)] (pro is proline). Yield, $721 \mathrm{mg}, 97 \%$. Anal. calcd for $\mathrm{C}_{35} \mathrm{H}_{28} \mathrm{IrN}_{3} \mathrm{O}_{2}$ : $\mathrm{C}_{5} 8.81, \mathrm{H} 3.95, \mathrm{~N} 5.88$; found: C 58.63, H 3.68, N 5.66\%. ESI-MS: m/z = 714.55 [M + H] ${ }^{+} .{ }^{1} \mathrm{H}$ NMR (400 MHz, $\left.\mathrm{CDCl}_{3}\right): 88.53(\mathrm{~d}, \mathrm{~J}=8.7 \mathrm{~Hz}, 1 \mathrm{H})$, $8.23(\mathrm{dd}, \mathrm{J}=14.9,8.8 \mathrm{~Hz}, 2 \mathrm{H}), 8.09(\mathrm{~d}, \mathrm{~J}=6.4 \mathrm{~Hz}, 1 \mathrm{H}), 8.07(\mathrm{~d}, \mathrm{~J}=6.5 \mathrm{~Hz}, 1 \mathrm{H}), 7.97-7.91(\mathrm{~m}, 1 \mathrm{H}), 7.86(\mathrm{~d}, \mathrm{~J}=7.8 \mathrm{~Hz}$, $1 \mathrm{H}), 7.83(\mathrm{~d}, \mathrm{~J}=7.9 \mathrm{~Hz}, 1 \mathrm{H}), 7.79(\mathrm{~d}, \mathrm{~J}=7.8 \mathrm{~Hz}, 1 \mathrm{H}), 7.76-7.71(\mathrm{~m}, 1 \mathrm{H}), 7.59-7.46(\mathrm{~m}, 4 \mathrm{H}), 7.01(\mathrm{t}, 1 \mathrm{H}), 6.96-6.88(\mathrm{~m}$, $2 \mathrm{H}), 6.73(\mathrm{t}, 1 \mathrm{H}), 6.58(\mathrm{t}, 1 \mathrm{H}), 6.29(\mathrm{~d}, \mathrm{~J}=7.6 \mathrm{~Hz}, 1 \mathrm{H}), 3.44(\mathrm{td}, 1 \mathrm{H}), 2.78(\mathrm{ddt}, 1 \mathrm{H}), 2.51(\mathrm{td}, 1 \mathrm{H}), 1.90(\mathrm{ddd}, \mathrm{J}=21.0$, 12.2, 9.o Hz, 1H), 1.63-1.48 (m, 3H), 1.32 (dd, J = 12.0, 7.1 Hz, 1H). ${ }^{13} \mathrm{C}$ NMR (101 MHz, $\mathrm{CDCl}_{3}$ ): $\delta$ 181.40, 172.26, 170.67, $151.44,150.23$, 149.67, 148.52, 146.50, 146.46, 138.90, 138.37, 137.46, 134.86, 132.12, 131.09, 130.25, 129.71, 129.31, 127.92, 127.82, 127.51, 126.90, 126.62, 126.21, 125.89, 123.03, 122.00, 120.93, 118.00, 116.13, 62.09, 48.78, 28.72, 26.00. $\mathrm{CD}\left(\Delta \varepsilon / \mathrm{M}^{-1} \mathrm{~cm}^{-1}, \mathrm{MeOH}\right): 264(+13), 293(-16), 313(-30), 354(-47), 466(+5)$.

For $\Delta-\left[\operatorname{Ir}(\mathbf{p q})_{2}\right.$ (gly)] (gly is glycine). Yield, $668 \mathrm{mg}$, 99\%. Anal. calcd for $\mathrm{C}_{32} \mathrm{H}_{24} \mathrm{IrN}_{3} \mathrm{O}_{2}:$ C 56.96, H 3.59, N 6.23; found: C 56.78, H 3.42, N 6.15\%. ESI-MS: m/z = 676.19 [M + H] ${ }^{+} .{ }^{1} \mathrm{H}$ NMR (400 MHz, CDCl 3 ): $88.66(\mathrm{~d}, \mathrm{~J}=8.8 \mathrm{~Hz}, 1 \mathrm{H})$, $8.23(\mathrm{~d}, \mathrm{~J}=9.0 \mathrm{~Hz}, 1 \mathrm{H}), 8.20(\mathrm{~d}, \mathrm{~J}=9.0 \mathrm{~Hz}, 1 \mathrm{H}), 8.10-8.03(\mathrm{~m}, 2 \mathrm{H}), 7.92-7.85(\mathrm{~m}, 2 \mathrm{H}), 7.84-7.78(\mathrm{~m}, 2 \mathrm{H}), 7.76(\mathrm{~d}, \mathrm{~J}=$ 7.9 Hz, 1H), 7.59-7.47 (m, 4H), 6.97 (t, 1H), 6.95-6.87 (m, 2H), 6.73-6.66 (m, 1H), 6.6o (t, 1H), 6.29 (d, J = 7.6 Hz, 1H), $3.28(\mathrm{dt}, 1 \mathrm{H}), 2.97(\mathrm{dd}, \mathrm{J}=15.0,7.8 \mathrm{~Hz}, 1 \mathrm{H}), 2.72(\mathrm{dt}, 1 \mathrm{H}), 2.5 \mathrm{O}(\mathrm{dq}, 1 \mathrm{H}) .{ }^{13} \mathrm{C} \mathrm{NMR}\left(101 \mathrm{MHz}, \mathrm{CDCl}_{3}\right): \delta 179.48,171.73$, 170.42, 150.82, 149.92, 149.14, 148.18, 146.53, 145.96, 138.95, 138.57, 137.08, 134.93, 132.29, 131.30, 130.08, 129.84, $129.60,127.96,127.57,127.35,127.01,126.31,126.22,125.99,123.83,122.05,121.08,117.74,116.54,46.04$.

For $\Delta-\left[\operatorname{Ir}(\mathbf{p q})_{2}\right.$ (D-pal)] (pal is phenylalanine). Yield, $749 \mathrm{mg}$, 98\%. Anal. calcd for $\mathrm{C}_{39} \mathrm{H}_{30} \mathrm{IrN}_{3} \mathrm{O}_{2}$ : C 61.24, $\mathrm{H} 3.95$, N 5.49; found: C 61.42, H 3.76, N 5.17\%. ESI-MS: $\mathrm{m} / \mathrm{z}=766.17[\mathrm{M}+\mathrm{H}]^{+} .{ }^{1} \mathrm{H}$ NMR (40o MHz, $\left.\mathrm{CDCl}_{3}\right): 88.74(\mathrm{~d}, \mathrm{~J}=8.7$ $\mathrm{Hz}, 1 \mathrm{H}), 8.21(\mathrm{dd}, \mathrm{J}=12.6,8.9 \mathrm{~Hz}, 2 \mathrm{H}), 8.05(\mathrm{dd}, \mathrm{J}=8.6,3.0 \mathrm{~Hz}, 2 \mathrm{H}), 7.88(\mathrm{~d}, \mathrm{~J}=8.0 \mathrm{~Hz}, 1 \mathrm{H}), 7.79(\mathrm{dt}, 4 \mathrm{H}), 7.61(\mathrm{t}, 1 \mathrm{H})$, $7.53(\mathrm{q}, 2 \mathrm{H}), 7.34(\mathrm{t}, 1 \mathrm{H}), 6.96(\mathrm{tq}, 5 \mathrm{H}), 6.85(\mathrm{~d}, \mathrm{~J}=7.7 \mathrm{~Hz}, 1 \mathrm{H}), 6.67(\mathrm{dd}, \mathrm{J}=16.8,7.4 \mathrm{~Hz}, 3 \mathrm{H}), 6.57(\mathrm{t}, 1 \mathrm{H}), 6.22(\mathrm{~d}, \mathrm{~J}=$ 7.6 Hz, 1H), 3.22-3.12 (m, 1H), 3.06 (dd, J = 10.9, 6.1 Hz, 1H), 2.95 (dd, J = 14.6, 3.6 Hz, 1H), 2.38 (dd, J = 14.5, 8.6 Hz, 1H), 2.17 (t, 1H). ${ }^{13} \mathrm{C} \mathrm{NMR} \mathrm{(101} \mathrm{MHz,} \mathrm{CDCl}_{3}$ ): $\delta$ 179.11, 171.92, 170.41, 151.27, 149.83, 148.88, 148.41, 146.46, 146.14, 138.93, 138.50, 137.0o, 136.85, 134.80, 132.13, 131.24, 129.94, 129.79, 129.57, 129.01, 128.67, 128.08, 127.97, 127.85, 127.00, 126.91, 126.43, 126.17, 125.93, 123.77, 121.99, 121.01, 117.80, 116.43, 56.53, 40.52. CD ( $\left.\Delta \varepsilon / \mathrm{M}^{-1} \mathrm{~cm}^{-1}, \mathrm{MeOH}\right)$ : 271(+47), 308(-57), 354(-54), 472(+9).

For $\boldsymbol{\Lambda}$-[Ir(Mpq) 2 (L-ala)]. Yield, $695 \mathrm{mg}, 97 \%$. Anal. calcd for $\mathrm{C}_{35} \mathrm{H}_{30} \mathrm{IrN}_{3} \mathrm{O}_{2}$ : C 58.64, H 4.22, N 5.86; found: C 58.49, H 4.30, N 5.61\%. ESI-MS: m/z = 718.08[M + H]+. ${ }^{1} \mathrm{H}$ NMR (400 MHz, $\left.\mathrm{CDCl}_{3}\right): 88.73(\mathrm{~s}, 1 \mathrm{H}), 8.22(\mathrm{~d}, \mathrm{~J}=8.7 \mathrm{~Hz}, 1 \mathrm{H})$, $8.11(\mathrm{~d}, \mathrm{~J}=8.7 \mathrm{~Hz}, 1 \mathrm{H}), 8.05(\mathrm{~d}, \mathrm{~J}=8.7 \mathrm{~Hz}, 1 \mathrm{H}), 7.98(\mathrm{~d}, \mathrm{~J}=8.7 \mathrm{~Hz}, 1 \mathrm{H}), 7.83(\mathrm{~d}, \mathrm{~J}=7.8 \mathrm{~Hz}, 1 \mathrm{H}), 7.79-7.70(\mathrm{~m}, 4 \mathrm{H})$, $6.96(\mathrm{t}, 1 \mathrm{H}), 6.89(\mathrm{t}, 2 \mathrm{H}), 6.69(\mathrm{t}, 1 \mathrm{H}), 6.58(\mathrm{t}, 1 \mathrm{H}), 6.25(\mathrm{~d}, \mathrm{~J}=7.8 \mathrm{~Hz}, 1 \mathrm{H}), 3.38(\mathrm{dd}, \mathrm{J}=11.0,6.4 \mathrm{~Hz}, 1 \mathrm{H}), 3.10(\mathrm{dq}, 1 \mathrm{H})$, $2.46(\mathrm{~s}, 3 \mathrm{H}), 2.44(\mathrm{~s}, 3 \mathrm{H}), 2.29-2.15(\mathrm{~m}, 1 \mathrm{H}), 0.95(\mathrm{~d}, \mathrm{~J}=7.0 \mathrm{~Hz}, 3 \mathrm{H}) .{ }^{13} \mathrm{C} \mathrm{NMR}\left(101 \mathrm{MHz}, \mathrm{CDCl}_{3}\right.$ ): $\delta$ 180.52, 171.68, 170.58, 150.87, 149.58, 149.22, 148.66, 146.61, 146.05, 143.48, 141.58, 138.70, 138.02, 136.79, 134.51, 129.77, 129.69, 129.45, $129.11,128.00,127.77,126.84,126.35,126.08,125.97,123.24,121.82,120.84,117.16,115.65,51.37,22.36,22.23 . \mathrm{CD}$ 
$\left(\Delta \varepsilon / \mathrm{M}^{-1} \mathrm{~cm}^{-1}, \mathrm{MeOH}\right): 270(-12), 309(+37), 355(+59), 479(-7)$.

For $\Lambda$-[Ir(Cpq) $)_{2}$ (L-ala)]. Yield, $742 \mathrm{mg}$, 98\%. Anal. calcd for $\mathrm{C}_{33} \mathrm{H}_{24} \mathrm{Cl}_{2} \mathrm{IrN}_{3} \mathrm{O}_{2}: \mathrm{C} 52.31, \mathrm{H} 3.19, \mathrm{~N}$ 5.55; found: C 52.14, H 3.42, N 5.31\%. ESI-MS: $\mathrm{m} / \mathrm{z}=758.12[\mathrm{M}+\mathrm{H}]^{+} .{ }^{1} \mathrm{H}$ NMR (400 MHz, $\mathrm{CDCl}_{3}$ ): $89.04(\mathrm{~s}, 1 \mathrm{H}), 8.26$ (d, J = 8.7 Hz, $1 \mathrm{H}), 8.13(\mathrm{t}, 2 \mathrm{H}), 8.04(\mathrm{~d}, \mathrm{~J}=8.8 \mathrm{~Hz}, 1 \mathrm{H}), 7.98(\mathrm{~s}, 1 \mathrm{H}), 7.81(\mathrm{tt}, 4 \mathrm{H}), 7.50(\mathrm{dd}, \mathrm{J}=12.4,9.0 \mathrm{~Hz}, 2 \mathrm{H}), 7.00(\mathrm{t}, 1 \mathrm{H}), 6.92(\mathrm{t}$, 1H), $6.86(\mathrm{~d}, \mathrm{~J}=7.7 \mathrm{~Hz}, 1 \mathrm{H}), 6.76(\mathrm{t}, 1 \mathrm{H}), 6.62(\mathrm{t}, 1 \mathrm{H}), 6.20(\mathrm{~d}, \mathrm{~J}=7.6 \mathrm{~Hz}, 1 \mathrm{H}), 3.35(\mathrm{dd}, \mathrm{J}=11.7,6.2 \mathrm{~Hz}, 1 \mathrm{H}), 3.11(\mathrm{dt}$, 1H), $2.23(\mathrm{t}, 1 \mathrm{H}), 1.05(\mathrm{~d}, \mathrm{~J}=6.9 \mathrm{~Hz}, 3 \mathrm{H}) .{ }^{13} \mathrm{C} \mathrm{NMR}\left(1 \mathrm{OHHz}, \mathrm{CDCl}_{3}\right): \delta$ 180.27, 172.89, 171.84, 151.25, 150.32, 149.37, 148.79, 146.04, 145.47, 139.05, 138.93, 138.12, 137.49, 136.58, 134.33, 131.26, 130.58, 130.16, 129.28, 128.11, 127.04, $126.80,126.76,126.69,126.26,123.37,122.22,121.34,118.24,116.77,51.47,22.41 . \mathrm{CD}\left(\Delta \varepsilon / \mathrm{M}^{-1} \mathrm{~cm}^{-1}, \mathrm{MeOH}\right): 269(-16)$, 311(+43), 359(+55), 483(-7).

For $\Lambda$-[Ir(Bpq) 2 (L-ala)]. Yield, $821.2 \mathrm{mg}$, 97\%. Anal. calcd for $\mathrm{C}_{33} \mathrm{H}_{24} \mathrm{Br}_{2} \mathrm{IrN}_{3} \mathrm{O}_{2}: \mathrm{C} 46.82, \mathrm{H}$ 2.86, N 4.96; found: C 46.93, H 2.91, N 4.74\%. ESI-MS: m/ z = 848.02 [M + H] ${ }^{+} .{ }^{1} \mathrm{H}$ NMR (400 MHz, $\mathrm{CDCl}_{3}$ ): $\delta 9.23(\mathrm{~s}, 1 \mathrm{H}), 8.25(\mathrm{~d}, \mathrm{~J}=8.8 \mathrm{~Hz}$, 1H), 8.19-8.12 (m, 3H), $8.05(\mathrm{~d}, \mathrm{~J}=8.8 \mathrm{~Hz}, 1 \mathrm{H}), 7.86(\mathrm{~d}, \mathrm{~J}=7.9 \mathrm{~Hz}, 1 \mathrm{H}), 7.74(\mathrm{t}, 3 \mathrm{H}), 7.67(\mathrm{~d}, \mathrm{~J}=8.5 \mathrm{~Hz}, 1 \mathrm{H}), 7.62(\mathrm{~d}, \mathrm{~J}$ $=8.4 \mathrm{~Hz}, 1 \mathrm{H}), 7.00(\mathrm{t}, 1 \mathrm{H}), 6.92(\mathrm{t}, 1 \mathrm{H}), 6.85(\mathrm{~d}, \mathrm{~J}=7.7 \mathrm{~Hz}, 1 \mathrm{H}), 6.76(\mathrm{t}, 1 \mathrm{H}), 6.62(\mathrm{t}, 1 \mathrm{H}), 6.19(\mathrm{~d}, \mathrm{~J}=7.6 \mathrm{~Hz}, 1 \mathrm{H}), 3.31$ (dd, J = 11.5, 6.1 Hz, 1H), 3.15 (dd, J = 11.4, $6.2 \mathrm{~Hz}, 1 \mathrm{H}), 2.25(\mathrm{t}, 1 \mathrm{H}), 1.08(\mathrm{~d}, \mathrm{~J}=6.9 \mathrm{~Hz}, 3 \mathrm{H}) .{ }^{13 \mathrm{C}} \mathrm{NMR}\left(101 \mathrm{MHz}, \mathrm{CDCl}_{3}\right)$ : $\delta$ 180.19, 172.87, 171.85, 151.21, 150.33, 149.43, 148.90, 146.01, 139.01, 138.17, 136.48, 134.28, 131.23, 130.76, 130.21, $129.92,129.33,129.26,127.43,127.06,126.79,126.56,126.53,125.69,122.21,121.35,118.41,116.91,51.50,22.51$. CD $\left(\Delta \varepsilon / \mathrm{M}^{-1} \mathrm{~cm}^{-1}, \mathrm{MeOH}\right): 244(-48), 308(+37), 359(+60), 500(-9)$.

\section{S2.4 General Procedure for the Synthesis of Ir(III) Imino acid Complexes.}

For $\boldsymbol{\Lambda}$-[Ir(pq) $\mathbf{2}(\mathbf{a l a - 2 H})]$ or $\boldsymbol{\Delta}$-[Ir(pq) $)_{2}\left(\right.$ ala-2H)]. Yield, $32 \mathrm{mg}, 93 \%$. Anal. calcd for $\mathrm{C}_{33} \mathrm{H}_{24} \mathrm{IrN}_{3} \mathrm{O}_{2}: \mathrm{C}$ 57.71, H 3.52, N 6.12; found: C 57.89, H 3.71, N 6.33\%. HR-MS anal. calcd for $\mathrm{C}_{33} \mathrm{H}_{25} \mathrm{IrN}_{3} \mathrm{O}_{2}\left(\mathrm{~m} / \mathrm{z}=[\mathrm{M}+\mathrm{H}]^{+}\right)$: 688.15705; found: 688.15670. ${ }^{1} \mathrm{H}$ NMR (400 MHz, $\mathrm{CDCl}_{3}$ ): $\delta 9.89(\mathrm{~s}, 1 \mathrm{H}), 8.59(\mathrm{~d}, \mathrm{~J}=8.6 \mathrm{~Hz}, 1 \mathrm{H}), 8.20(\mathrm{~d}, \mathrm{~J}=5.2 \mathrm{~Hz}, 1 \mathrm{H}), 8.18(\mathrm{~d}, \mathrm{~J}=5.1$ $\mathrm{Hz}, 1 \mathrm{H}), 8.05(\mathrm{~d}, \mathrm{~J}=8.7 \mathrm{~Hz}, 2 \mathrm{H}), 7.85(\mathrm{~d}, \mathrm{~J}=7.7 \mathrm{~Hz}, 1 \mathrm{H}), 7.82-7.76(\mathrm{~m}, 3 \mathrm{H}), 7.65(\mathrm{~d}, \mathrm{~J}=8.7 \mathrm{~Hz}, 1 \mathrm{H}), 7.5 \mathrm{O}(\mathrm{ddd}, \mathrm{J}=11.5$, 7.9, $6.3 \mathrm{~Hz}, 2 \mathrm{H}), 7.47-7.39(\mathrm{~m}, 1 \mathrm{H}), 7.36(\mathrm{t}, 1 \mathrm{H}), 6.99(\mathrm{t}, 1 \mathrm{H}), 6.93(\mathrm{t}, 1 \mathrm{H}), 6.81(\mathrm{~d}, \mathrm{~J}=7.7 \mathrm{~Hz}, 1 \mathrm{H}), 6.7 \mathrm{O}(\mathrm{t}, 1 \mathrm{H}), 6.63(\mathrm{t}$, $1 \mathrm{H}), 6.29(\mathrm{~d}, \mathrm{~J}=7.6 \mathrm{~Hz}, 1 \mathrm{H}), 1.89-1.85(\mathrm{~m}, 4 \mathrm{H}), 1.87(\mathrm{~d}, \mathrm{~J}=1.5 \mathrm{~Hz}, 3 \mathrm{H}) .{ }^{13 \mathrm{C}} \mathrm{NMR}\left(101 \mathrm{MHz}, \mathrm{CDCl}_{3}\right.$ ): $\delta$ 177.56, 171.33, 171.11, 169.62, 152.09, 148.34, 148.16, 146.83, 146.77, 145.92, 138.70, 138.67, 136.37, 134.86, 131.89, 130.01, 129.82, $129.65,129.18,128.26$, 127.92, 127.79, 127.76, 127.12, 126.72, 126.14, 126.04, 125.93, 122.06, 121.09, 117.37, 116.19, 24.41. $\mathrm{CD}\left(\Delta \varepsilon / \mathrm{M}^{-1} \mathrm{~cm}^{-1}, \mathrm{MeOH}\right)$ for $\Lambda$-[Ir(pq) $)_{2}\left(\right.$ ala-2H)]: 268(-47), 305(+57), 352(+53), 466(-15). $\mathrm{CD}\left(\Delta \varepsilon / \mathrm{M}^{-1} \mathrm{~cm}^{-1}, \mathrm{MeOH}\right)$ for $\Delta$-[Ir(pq) 2 (ala-2H)]: 268(+39), 305(-49), 352(-46), 466(+12).

\section{S2.5 General Procedure for the C-N Cross Coupling Reaction.}

For $\boldsymbol{\Lambda}$-Ir(pq)(L-pqa) or $\boldsymbol{\Delta}$-Ir(pq)(D-pqa) (pqa $=\mathrm{N}$-(2-phenylquinolin-8-yl)alanine). Reaction time is $18 \mathrm{~h}$. Isolated yield, $30.2 \mathrm{mg}$, 88\%. Anal. calcd for $\mathrm{C}_{33} \mathrm{H}_{24} \mathrm{IrN}_{3} \mathrm{O}_{2}$ : $\mathrm{C} 57.71, \mathrm{H} 3.52, \mathrm{~N} 6.12$; found: $\mathrm{C} 57.73, \mathrm{H} 3.53, \mathrm{~N} 6.14 \%$. HR- 
MS anal. calcd for $\mathrm{C}_{33} \mathrm{H}_{25} \mathrm{IrN}_{3} \mathrm{O}_{2}\left(\mathrm{~m} / \mathrm{z}=[\mathrm{M}+\mathrm{H}]+{ }^{+}\right): 688.15705$; found: 688.15452. ${ }^{1} \mathrm{H}$ NMR (400 MHz, $\left.\mathrm{CDCl}_{3}\right): \delta 9.21(\mathrm{~d}$, $\mathrm{J}=8.8 \mathrm{~Hz}, 1 \mathrm{H}), 8.24(\mathrm{dd}, \mathrm{J}=14.0,8.8 \mathrm{~Hz}, 2 \mathrm{H}), 8.06(\mathrm{~d}, \mathrm{~J}=8.9 \mathrm{~Hz}, 2 \mathrm{H}), 7.91(\mathrm{~d}, \mathrm{~J}=7.1 \mathrm{~Hz}, 1 \mathrm{H}), 7.87-7.8 \mathrm{o}(\mathrm{m}, 2 \mathrm{H}), 7.78$ $(\mathrm{d}, \mathrm{J}=2.8 \mathrm{~Hz}, 1 \mathrm{H}), 7.76(\mathrm{~d}, \mathrm{~J}=2.8 \mathrm{~Hz}, 1 \mathrm{H}), 7.62(\mathrm{t}, 1 \mathrm{H}), 7.52(\mathrm{t}, 1 \mathrm{H}), 7.46(\mathrm{~d}, \mathrm{~J}=7.4 \mathrm{~Hz}, 1 \mathrm{H}), 6.91(\mathrm{t}, 1 \mathrm{H}), 6.86(\mathrm{t}, 1 \mathrm{H})$, 6.72-6.65 (m, 1H), $6.55(\mathrm{t}, 1 \mathrm{H}), 6.46(\mathrm{~d}, \mathrm{~J}=7.6 \mathrm{~Hz}, 1 \mathrm{H}), 5.74(\mathrm{~d}, \mathrm{~J}=7.4 \mathrm{~Hz}, 1 \mathrm{H}), 3.97(\mathrm{q}, 1 \mathrm{H}), 0.81(\mathrm{~d}, \mathrm{~J}=7.1 \mathrm{~Hz}, 3 \mathrm{H}) .{ }^{13} \mathrm{C}$ NMR (101 MHz, $\left.\mathrm{CDCl}_{3}\right): \delta$ 181.02, 168.34, 166.47, 149.40, 147.91, 147.84, 145.86, 145.62, 144.49, 137.84, 135.93, 134.39, $132.18,131.82,130.81,129.70,129.33,128.41,128.01,127.97,127.93,127.89,127.05,126.83,125.68,121.38,117.85,116.44$, 67.32, 18.04. $\mathrm{CD}\left(\Delta \varepsilon / \mathrm{M}^{-1} \mathrm{~cm}^{-1}, \mathrm{MeOH}\right)$ for $\Lambda$-[Ir(pq)(L-pqa)]: 261(-24), 290 $(+15), 313(+35), 353(+46), 463(-8) . \mathrm{CD}$ $\left(\Delta \varepsilon / \mathrm{M}^{-1} \mathrm{~cm}^{-1}, \mathrm{MeOH}\right)$ for $\Delta$-Ir(pq)(D-pqa): 262(+22), 290(-18), 312(+31), 354(-44), 463(+8).

For $\Delta$-[Ir(pq)(D-pqv)] (pqv is N-(2-phenylquinolin-8-yl)valine). Reaction time is $60 \mathrm{~h}$ in the presence of $3 \mathrm{eq}$ NaOMe. Isolated yield, $29.6 \mathrm{mg}, 83 \%$. Anal. calcd for $\mathrm{C}_{35} \mathrm{H}_{28} \mathrm{IrN}_{3} \mathrm{O}_{2}$ : C $58.81, \mathrm{H} 3.95, \mathrm{~N} 5.88$; found: $\mathrm{C} 58.64, \mathrm{H} 3.72, \mathrm{~N}$ 5.69\%. HR-MS anal. calcd for $\mathrm{C}_{35} \mathrm{H}_{29} \mathrm{IrN}_{3} \mathrm{O}_{2}\left(\mathrm{~m} / \mathrm{z}=[\mathrm{M}+\mathrm{H}]^{+}\right)$: 716.18835 ; found: $716.18606 .{ }^{1} \mathrm{H}$ NMR (400 MHz, $\left.\mathrm{CDCl}_{3}\right)$ : $\delta 9.18(\mathrm{~d}, \mathrm{~J}=8.9 \mathrm{~Hz}, 1 \mathrm{H}), 8.26(\mathrm{~d}, \mathrm{~J}=8.7 \mathrm{~Hz}, 1 \mathrm{H}), 8.21(\mathrm{~d}, \mathrm{~J}=8.9 \mathrm{~Hz}, 1 \mathrm{H}), 8.05(\mathrm{~d}, \mathrm{~J}=8.8 \mathrm{~Hz}, 2 \mathrm{H}), 7.91(\mathrm{~d}, \mathrm{~J}=8.1 \mathrm{~Hz}$, 1H), $7.79(\mathrm{dd}, \mathrm{J}=22.0,8.0 \mathrm{~Hz}, 4 \mathrm{H}), 7.61(\mathrm{t}, 1 \mathrm{H}), 7.51(\mathrm{t}, 1 \mathrm{H}), 7.40(\mathrm{~d}, \mathrm{~J}=7.5 \mathrm{~Hz}, 1 \mathrm{H}), 6.88(\mathrm{dt}, 2 \mathrm{H}), 6.69(\mathrm{t}, 1 \mathrm{H}), 6.56(\mathrm{t}$, 1H), $6.47(\mathrm{~d}, \mathrm{~J}=7.7 \mathrm{~Hz}, 1 \mathrm{H}), 5.73(\mathrm{~d}, \mathrm{~J}=7.7 \mathrm{~Hz}, 1 \mathrm{H}), 4.47(\mathrm{~s}, 1 \mathrm{H}), 3.37(\mathrm{~d}, \mathrm{~J}=9.5 \mathrm{~Hz}, 1 \mathrm{H}), 0.66(\mathrm{~d}, \mathrm{~J}=6.6 \mathrm{~Hz}, 3 \mathrm{H}), 0.58$ (d, J = $6.8 \mathrm{~Hz}, 3 \mathrm{H}) .{ }^{13} \mathrm{C} \mathrm{NMR}\left(101 \mathrm{MHz}, \mathrm{CDCl}_{3}\right): \delta$ 166.56, 149.37, 148.07, 147.83, 145.84, 145.52, 144.24, 137.81, 135.95, $134.38,131.94,131.82$, 130.81, 129.72, 129.22, 128.52, 128.47, 127.92, 127.86, 127.00, 126.85, 125.82, 121.39, 121.34, 117.97, 116.33, 53.56, 29.52, 20.57, 19.59. $\mathrm{CD}\left(\Delta \varepsilon / \mathrm{M}^{-1} \mathrm{~cm}^{-1}, \mathrm{MeOH}\right): 263(+22), 275(-7)$, 291(-11), 314(-27), 354(-41).

For $\Delta$-[Ir(pq)(D-pqs)] (pqs is $\mathrm{N}$-(2-phenylquinolin-8-yl)serine). Reaction time is $60 \mathrm{~h}$ in the presence of 3 eq NaOMe. Isolated yield, $18.6 \mathrm{mg}, 53 \%$. Anal. calcd for $\mathrm{C}_{33} \mathrm{H}_{24} \mathrm{IrN}_{3} \mathrm{O}_{3}: \mathrm{C} 56.40, \mathrm{H} 3.44, \mathrm{~N} 5.98$; found: $\mathrm{C} 56.22, \mathrm{H} 3.25, \mathrm{~N}$ 5.76\%. HR-MS anal. calcd for $\mathrm{C}_{33} \mathrm{H}_{25} \mathrm{IrN}_{3} \mathrm{O}_{3}\left(\mathrm{~m} / \mathrm{z}=[\mathrm{M}+\mathrm{H}]^{+}\right)$: 704.15197; found: 704.14943. ${ }^{1} \mathrm{H}$ NMR (400 MHz, $\left.\mathrm{CDCl}_{3}\right)$ : $\delta 8.97(\mathrm{~d}, \mathrm{~J}=8.8 \mathrm{~Hz}, 1 \mathrm{H}), 8.15(\mathrm{dd}, \mathrm{J}=13.4,8.9 \mathrm{~Hz}, 2 \mathrm{H}), 7.99(\mathrm{dd}, \mathrm{J}=17.2,8.8 \mathrm{~Hz}, 2 \mathrm{H}), 7.82(\mathrm{~d}, \mathrm{~J}=8.0 \mathrm{~Hz}, 1 \mathrm{H}), 7.75$ $(\mathrm{d}, \mathrm{J}=7.7 \mathrm{~Hz}, 1 \mathrm{H}), 7.68(\mathrm{q}, 3 \mathrm{H}), 7.50(\mathrm{t}, 1 \mathrm{H}), 7.27(\mathrm{q}, 3 \mathrm{H}), 6.90(\mathrm{t}, 1 \mathrm{H}), 6.8 \mathrm{o}(\mathrm{t}, 1 \mathrm{H}), 6.68(\mathrm{t}, 1 \mathrm{H}), 6.49(\mathrm{q}, 2 \mathrm{H}), 5.61(\mathrm{~d}, \mathrm{~J}$ = $7.7 \mathrm{~Hz}, 1 \mathrm{H}), 3.81(\mathrm{~d}, \mathrm{~J}=10.5 \mathrm{~Hz}, 1 \mathrm{H}), 3.24(\mathrm{~d}, \mathrm{~J}=9.2 \mathrm{~Hz}, 1 \mathrm{H}), 2.34(\mathrm{t}, 1 \mathrm{H}) .{ }^{13} \mathrm{C} \mathrm{NMR}\left(101 \mathrm{MHz}, \mathrm{CDCl}_{3}\right): \delta$ 179.07, 168.51, 166.40, 148.99, 148.25, 146.97, 145.98, 145.90, 145.43, 145.05, 137.81, 135.93, 134.61, 131.90, 131.27, 130.80, 129.58, 129.36, 128.27, 128.23, 128.03, 127.93, 127.78, 127.35, 126.84, 126.79, 125.97, 121.43, 121.41, 117.77, 116.75, 74.80, 61.05 . $\mathrm{CD}\left(\Delta \varepsilon / \mathrm{M}^{-1} \mathrm{~cm}^{-1}, \mathrm{MeOH}\right): 260(+25), 290(-17), 313(-31), 353(-45)$.

For $\Delta$-[Ir(pq)(D-pqp)] (pqp is N-(2-phenylquinolin-8-yl)proline). Reaction time is $50 \mathrm{~h}$. Isolated yield, $25.7 \mathrm{mg}$, 72\%. Anal. calcd for $\mathrm{C}_{35} \mathrm{H}_{26} \mathrm{IrN}_{3} \mathrm{O}_{2}$ : C 58.97, $\mathrm{H}$ 3.68, N 5.89; found: $\mathrm{C} 58.69, \mathrm{H} 3.42, \mathrm{~N}$ 5.55\%. HR-MS anal. calcd for $\mathrm{C}_{35} \mathrm{H}_{27} \mathrm{IrN}_{3} \mathrm{O}_{2}\left(\mathrm{~m} / \mathrm{z}=[\mathrm{M}+\mathrm{H}]^{+}\right): 714.1727 \mathrm{O}$; found: 714.17006. ${ }^{1} \mathrm{H} \mathrm{NMR}\left(400 \mathrm{MHz}, \mathrm{CDCl}_{3}\right): \delta 9.33(\mathrm{~d}, \mathrm{~J}=8.8 \mathrm{~Hz}, 1 \mathrm{H})$, 8.27 (dd, J = 15.0, $8.8 \mathrm{~Hz}, 2 \mathrm{H}), 8.14-8.03(\mathrm{~m}, 2 \mathrm{H}), 7.95(\mathrm{~d}, \mathrm{~J}=7.9 \mathrm{~Hz}, 1 \mathrm{H}), 7.86(\mathrm{~d}, \mathrm{~J}=7.4 \mathrm{~Hz}, 2 \mathrm{H}), 7.77$ (d, J = 7.7 Hz, 2H), 7.71-7.55 (m, 3H), $6.90(\mathrm{q}, 2 \mathrm{H}), 6.68(\mathrm{t}, 1 \mathrm{H}), 6.60(\mathrm{t}, 1 \mathrm{H}), 6.35(\mathrm{~d}, \mathrm{~J}=7.5 \mathrm{~Hz}, 1 \mathrm{H}), 5.85(\mathrm{~d}, \mathrm{~J}=7.6 \mathrm{~Hz}, 1 \mathrm{H}), 4.00(\mathrm{t}$, 1H), $3.80-3.63(\mathrm{~m}, 1 \mathrm{H}), 3.39(\mathrm{q}, 1 \mathrm{H}), 2.74(\mathrm{t}, 1 \mathrm{H}), 2.26-1.98(\mathrm{~m}, 2 \mathrm{H}), 0.76(\mathrm{dt}, 1 \mathrm{H}) .{ }^{13} \mathrm{C}$ NMR $\left(101 \mathrm{MHz}, \mathrm{CDCl}_{3}\right): \delta$ 168.57, 
$166.41,149.66,149.38,146.16,146.00,145.81,144.85,138.06,136.22,134.21,132.38,131.59,130.79,130.02,128.64$, 128.00, 127.79, 127.65, 127.09, 126.76, 126.04, 125.67, 121.45, 121.31, 117.79, 116.44, 78.99, 59.99, 53.56, 29.85, 23.49. $\mathrm{CD}\left(\Delta \varepsilon / \mathrm{M}^{-1} \mathrm{~cm}^{-1}, \mathrm{MeOH}\right): 261(+18), 273(-12), 291(-11), 315(-27), 353(-50)$.

For $\Delta$-[Ir(pq)(pqg)] (pqg is N-(2-phenylquinolin-8-yl)glycine). Reaction time is $60 \mathrm{~h}$. Isolated yield, $22.9 \mathrm{mg}, 68 \%$. Anal. calcd for $\mathrm{C}_{32} \mathrm{H}_{22} \mathrm{IrN}_{3} \mathrm{O}_{2}$ : C 57.13, $\mathrm{H}$ 3.30, $\mathrm{N}$ 6.25; found: C 57.34, H 3.48, N 6.43\%. HR-MS anal. calcd for $\mathrm{C}_{32} \mathrm{H}_{23} \mathrm{IrN}_{3} \mathrm{O}_{2}\left(\mathrm{~m} / \mathrm{z}=[\mathrm{M}+\mathrm{H}]^{+}\right): 674.14140$; found: 674.13888. ${ }^{1} \mathrm{H}$ NMR $\left(400 \mathrm{MHz}, \mathrm{CDCl}_{3}\right): \delta 9.16(\mathrm{~d}, \mathrm{~J}=8.4 \mathrm{~Hz}, 1 \mathrm{H})$, $8.24(\mathrm{~d}, \mathrm{~J}=8.7 \mathrm{~Hz}, 1 \mathrm{H}), 8.18(\mathrm{~d}, \mathrm{~J}=8.9 \mathrm{~Hz}, 1 \mathrm{H}), 8.01(\mathrm{dt}, 2 \mathrm{H}), 7.90(\mathrm{~d}, \mathrm{~J}=7.8 \mathrm{~Hz}, 1 \mathrm{H}), 7.78(\mathrm{dd}, \mathrm{J}=13.0,7.9 \mathrm{~Hz}, 3 \mathrm{H})$, 7.73-7.65 (m, 1H), 7.59 (t, 1H), $7.40(\mathrm{dt}, 2 \mathrm{H}), 6.90(\mathrm{t}, 1 \mathrm{H}), 6.81(\mathrm{q}, 1 \mathrm{H}), 6.7 \mathrm{O}(\mathrm{t}, 1 \mathrm{H}), 6.54(\mathrm{t}, 2 \mathrm{H}), 5.81(\mathrm{~d}, \mathrm{~J}=7.6 \mathrm{~Hz}, 1 \mathrm{H})$, $3.89(\mathrm{dd}, \mathrm{J}=15.0,8.1 \mathrm{~Hz}, 1 \mathrm{H}), 3.34$ (td, $1 \mathrm{H}) .{ }^{13} \mathrm{C} \mathrm{NMR}\left(101 \mathrm{MHz}, \mathrm{CDCl}_{3}\right):$ : $168.25,149.37,148.59,146.03,145.43,144.25$, 137.80, 135.98, 134.93, 132.21, 131.81, 130.96, 129.66, 128.97, 128.37, 128.01, 127.95, 127.86, 127.80, 127.08, 126.89, $125.64,121.40,117.93,116.51,59.70$.

For $\Delta$-[Ir(pq)(D-pqpa)] (pqpa is $\mathrm{N}$-(2-phenylquinolin-8-yl)phenylalanine). Reaction time is $72 \mathrm{~h}$ in the presence of 3eq NaOMe. Isolated yield, $23.6 \mathrm{mg}, 62 \%$. Anal. calcd for $\mathrm{C}_{39} \mathrm{H}_{28} \mathrm{IrN}_{3} \mathrm{O}_{2}$ : C 61.40, H 3.70, N 5.51; found: C 61.29, $\mathrm{H}$ 3.94, $\mathrm{N}$ 5.32\%. HR-MS anal. calcd for $\mathrm{C}_{39} \mathrm{H}_{28} \mathrm{IrN}_{3} \mathrm{O}_{2}\left(\mathrm{~m} / \mathrm{z}=[\mathrm{M}+\mathrm{H}]^{+}\right)$: 764.18835; found: 764.18596. ${ }^{1} \mathrm{H}$ NMR (40o MHz, $\left.\mathrm{CDCl}_{3}\right): \delta 9.28(\mathrm{~d}, \mathrm{~J}=8.3 \mathrm{~Hz}, 1 \mathrm{H}), 8.36(\mathrm{~d}, \mathrm{~J}=8.7 \mathrm{~Hz}, 1 \mathrm{H}), 8.20(\mathrm{~d}, \mathrm{~J}=10.6 \mathrm{~Hz}, 2 \mathrm{H}), 8.05(\mathrm{~d}, \mathrm{~J}=8.9 \mathrm{~Hz}, 1 \mathrm{H}), 7.95(\mathrm{~d}, \mathrm{~J}$ $=7.9 \mathrm{~Hz}, 1 \mathrm{H}), 7.89(\mathrm{t}, 1 \mathrm{H}), 7.79(\mathrm{dd}, \mathrm{J}=16.7,8.0 \mathrm{~Hz}, 3 \mathrm{H}), 7.65(\mathrm{t}, 1 \mathrm{H}), 7.39(\mathrm{t}, 1 \mathrm{H}), 7.17(\mathrm{dq}, 3 \mathrm{H}), 6.88$ (ddd, J = 30.3, 15.0, $7.4 \mathrm{~Hz}, 3 \mathrm{H}), 6.72(\mathrm{t}, 1 \mathrm{H}), 6.58(\mathrm{~d}, \mathrm{~J}=6.9 \mathrm{~Hz}, 2 \mathrm{H}), 6.51(\mathrm{dd}, \mathrm{J}=16.4,7.9 \mathrm{~Hz}, 2 \mathrm{H}), 5.64(\mathrm{~d}, \mathrm{~J}=7.6 \mathrm{~Hz}, 1 \mathrm{H}), 4.03(\mathrm{~d}, \mathrm{~J}$ $=12.4 \mathrm{~Hz}, 1 \mathrm{H}), 2.94(\mathrm{~d}, \mathrm{~J}=14.1 \mathrm{~Hz}, 1 \mathrm{H}), 1.77(\mathrm{t}, 1 \mathrm{H}) .{ }^{13} \mathrm{C} N M R\left(101 \mathrm{MHz}, \mathrm{CDCl}_{3}\right):$ 179.72, 168.79, 166.50, 149.59, 147.95, $145.87,144.52$, 138.17, 136.43, 135.97, 134.51, 132.52, 131.53, 130.87, 129.69, 129.56, 128.91, 128.88, 128.34, 128.13, $128.03,127.95,127.75,127.66,127.28,126.88,125.76,121.52,121.47,117.91,116.40,73.34,37.56 . \mathrm{CD}\left(\Delta \varepsilon / \mathrm{M}^{-1} \mathrm{~cm}^{-1}\right.$, MeOH): 262(+23), 291(-16), 313(-36), 353(-49).

For $\Lambda$-[Ir(Mpq)(L-Mpqa)] (Mpqa is N-(7-methyl-2-phenylquinolin-8-yl)alanine). Reaction time is $22 \mathrm{~h}$. Isolated yield, $31.8 \mathrm{mg}$, 89\%. Anal. calcd for $\mathrm{C}_{35} \mathrm{H}_{28} \mathrm{IrN}_{3} \mathrm{O}_{2}$ : C 58.81, H 3.95, N 5.88; found: $\mathrm{C} 58.64, \mathrm{H} 3.73$, N 5.64\%. HR-MS anal. calcd for $\mathrm{C}_{35} \mathrm{H}_{29} \mathrm{IrN}_{3} \mathrm{O}_{2}(\mathrm{~m} / \mathrm{z}=[\mathrm{M}+\mathrm{H}]+)$ ): 716.18835; found: 716.18582. ${ }^{1} \mathrm{H}$ NMR (400 MHz, $\left.\mathrm{CDCl}_{3}\right): \delta 9.08(\mathrm{~s}, 1 \mathrm{H}), 8.20$ (d, J = 8.6 Hz, 1H), $8.13(\mathrm{~d}, \mathrm{~J}=8.8 \mathrm{~Hz}, 1 \mathrm{H}), 7.98(\mathrm{dd}, \mathrm{J}=8.5,5.9 \mathrm{~Hz}, 2 \mathrm{H}), 7.80(\mathrm{~d}, \mathrm{~J}=8.2 \mathrm{~Hz}, 1 \mathrm{H}), 7.73(\mathrm{t}, 3 \mathrm{H}), 7.44(\mathrm{~d}$, $\mathrm{J}=8.2 \mathrm{~Hz}, 1 \mathrm{H}), 7.35(\mathrm{~d}, \mathrm{~J}=8.3 \mathrm{~Hz}, 1 \mathrm{H}), 6.88(\mathrm{t}, 1 \mathrm{H}), 6.83(\mathrm{t}, 1 \mathrm{H}), 6.68(\mathrm{t}, 1 \mathrm{H}), 6.52(\mathrm{t}, 1 \mathrm{H}), 6.45(\mathrm{~d}, \mathrm{~J}=7.6 \mathrm{~Hz}, 1 \mathrm{H}), 5.70$ $(\mathrm{d}, \mathrm{J}=7.6 \mathrm{~Hz}, 1 \mathrm{H}), 3.82(\mathrm{q}, 1 \mathrm{H}), 2.58(\mathrm{~s}, 3 \mathrm{H}), 2.37(\mathrm{~s}, 3 \mathrm{H}), 0.85(\mathrm{~d}, \mathrm{~J}=7.1 \mathrm{~Hz}, 3 \mathrm{H}) .{ }^{13} \mathrm{C} \mathrm{NMR}\left(101 \mathrm{MHz}, \mathrm{CDCl}_{3}\right): \delta 181.01$, $168.18,166.43,149.48,148.02,147.81,145.90,145.72,145.59,142.79,141.56,137.88,137.31,135.52,134.21,131.77$, $130.94,130.56,129.44,128.97,127.45,127.42,127.25,126.85,126.54,125.87,125.40,121.17,121.05,116.92,115.43,64.12$, 31.60, 22.31, 17.72. $\mathrm{CD}\left(\Delta \varepsilon / \mathrm{M}^{-1} \mathrm{~cm}^{-1}, \mathrm{MeOH}\right): 262(-20), 292(+8), 314(+31), 353(+54), 465(-6)$.

For $\boldsymbol{\Lambda}$-[Ir(Cpq)(L-Cpqa)] (Cpqa is N-(7-chloro-2-phenylquinolin-8-yl)alanine). Reaction time is $28 \mathrm{~h}$. Isolated yield, $33.6 \mathrm{mg}, 89 \%$. Anal. calcd for $\mathrm{C}_{33} \mathrm{H}_{22} \mathrm{Cl}_{2} \mathrm{IrN}_{3} \mathrm{O}_{2}$ : $\mathrm{C} 52.45, \mathrm{H}$ 2.93, $\mathrm{N}$ 5.56; found: $\mathrm{C} 52.27, \mathrm{H} 2.61, \mathrm{~N}$ 5.33\%. HR-MS 
anal. calcd for $\mathrm{C}_{33} \mathrm{H}_{23} \mathrm{Cl}_{2} \mathrm{IrN}_{3} \mathrm{O}_{2}\left(\mathrm{~m} / \mathrm{z}=[\mathrm{M}+\mathrm{H}]+{ }^{+}\right): 756.07911$; found: 756.07719. ${ }^{1} \mathrm{H} \mathrm{NMR}\left(400 \mathrm{MHz}, \mathrm{CDCl}_{3}\right): \delta 9.37(\mathrm{~s}$, $1 \mathrm{H}), 8.24(\mathrm{~d}, \mathrm{~J}=8.6 \mathrm{~Hz}, 1 \mathrm{H}), 8.17(\mathrm{~d}, \mathrm{~J}=8.9 \mathrm{~Hz}, 1 \mathrm{H}), 8.06(\mathrm{dd}, \mathrm{J}=12.1,8.9 \mathrm{~Hz}, 2 \mathrm{H}), 7.88-7.74(\mathrm{~m}, 4 \mathrm{H}), 7.59$ (dd, J = 8.6, 1.9 Hz, 1H), 7.53 (d, J = 8.7 Hz, 1H), $6.90(\mathrm{dt}, 2 \mathrm{H}), 6.71(\mathrm{t}, 1 \mathrm{H}), 6.57(\mathrm{t}, 1 \mathrm{H}), 6.4 \mathrm{O}(\mathrm{d}, \mathrm{J}=7.3 \mathrm{~Hz}, 1 \mathrm{H}), 5.7 \mathrm{O}(\mathrm{d}, \mathrm{J}=7.4$ $\mathrm{Hz}, 1 \mathrm{H}), 4.06$ (q, 1H), 0.91 (d, J = 7.1 Hz, $3 \mathrm{H}) .{ }^{13} \mathrm{C} \mathrm{NMR}\left(101 \mathrm{MHz}, \mathrm{CDCl}_{3}\right.$ ): $\delta$ 181.06, 169.80, 167.69, 149.91, 148.75, 147.70, $146.47,145.61$, 145.40, 140.90, 138.68, 137.89, 136.22, 135.08, 134.25, 132.02, 131.50, 130.42, 129.38, 129.34, 129.24, $128.25,127.58,127.36,127.26,126.52,126.42,121.87,121.83,118.23,116.91,63.83,18.24 . \mathrm{CD}\left(\Delta \varepsilon / \mathrm{M}^{-1} \mathrm{~cm}^{-1}, \mathrm{MeOH}\right)$ : 266(-22), 304(-8), 318(+14), 357(+22).

For $\boldsymbol{\Lambda}$-[Ir(Bpq)(L-Bpqa)] (Bpqa is N-(7-bromo-2-phenylquinolin-8-yl)alanine). Reaction time is $27 \mathrm{~h}$. Isolated yield, $36.3 \mathrm{mg}, 86 \%$. Anal. calcd for $\mathrm{C}_{33} \mathrm{H}_{22} \mathrm{Br}_{2} \mathrm{IrN}_{3} \mathrm{O}_{2}: \mathrm{C} 46.93, \mathrm{H}$ 2.63, $\mathrm{N}$ 4.98; found: $\mathrm{C} 46.75, \mathrm{H}$ 2.84, N 4.72\%. HR-MS anal. calcd for $\mathrm{C}_{33} \mathrm{H}_{22} \mathrm{Br}_{2} \mathrm{IrN}_{3} \mathrm{O}_{2}\left(\mathrm{~m} / \mathrm{z}=[\mathrm{M}+\mathrm{H}]+\right.$ ): 845.97603; found: 845.97210. ${ }^{1} \mathrm{H}$ NMR (400 MHz, $\left.\mathrm{CDCl}_{3}\right)$ : $\delta 9.55$ (s, $1 \mathrm{H}), 8.23(\mathrm{~d}, \mathrm{~J}=8.8 \mathrm{~Hz}, 1 \mathrm{H}), 8.16(\mathrm{~d}, \mathrm{~J}=8.7 \mathrm{~Hz}, 1 \mathrm{H}), 8.07(\mathrm{dd}, \mathrm{J}=15.5,8.8 \mathrm{~Hz}, 2 \mathrm{H}), 7.80-7.66(\mathrm{~m}, 6 \mathrm{H}), 6.97-6.82(\mathrm{~m}$, 2H), $6.71(\mathrm{t}, 1 \mathrm{H}), 6.56(\mathrm{t}, 1 \mathrm{H}), 6.38(\mathrm{~d}, \mathrm{~J}=7.7 \mathrm{~Hz}, 1 \mathrm{H}), 5.68(\mathrm{~d}, \mathrm{~J}=7.8 \mathrm{~Hz}, 1 \mathrm{H}), 4.07(\mathrm{~d}, \mathrm{~J}=7.1 \mathrm{~Hz}, 1 \mathrm{H}), 0.93(\mathrm{~d}, \mathrm{~J}=7.1$ $\mathrm{Hz}, 3 \mathrm{H}$ ). ${ }^{13 \mathrm{C}} \mathrm{NMR}\left(1 \mathrm{O} 1 \mathrm{MHz}, \mathrm{CDCl}_{3}\right.$ ): $\delta$ 180.61, 169.62, 167.62, 149.80, 148.74, 147.57, 146.25, 145.31, 145.17, 142.49, 137.73, 135.92, 134.01, 131.84, 131.39, 130.68, 130.45, 130.26, 129.11, 129.05, 127.56, 127.37, 126.87, 126.57, 126.25, 125.33, 121.69, 121.62, 118.18, 116.83, 63.90, 18.21. $\mathrm{CD}\left(\Delta \varepsilon / \mathrm{M}^{-1} \mathrm{~cm}^{-1}, \mathrm{MeOH}\right): 242(-62), 265(-36), 317(+33), 358(+55)$, $480(-6)$.

\section{S3. For Nitrogen Radical Trapping Experiments.}

$\Lambda$ - $\operatorname{Ir}(\mathrm{pq})_{2}(\mathrm{~L}-\mathrm{ala})(0.005 \mathrm{mmol})$ and BHT $(0.01 \mathrm{mmol})$ were dissolved in $10 \mathrm{~mL}$ of ethanol in a $25 \mathrm{~mL}$ glass vessel equipped with a $\mathrm{O}_{2}$ balloon. The solution was stirred at $60{ }^{\circ} \mathrm{C}$ under irradiated with $10 \mathrm{~W}$ blue LEDs $(\lambda=450-470 \mathrm{~nm})$ for $18 \mathrm{~h}$. After that, the reaction mixtures were analyzed by HRMS.

\section{S4. For Carbon Radical Trapping Experiments.}

$\Lambda$-Ir(pq) $)_{2}(\mathrm{~L}$-ala) $(0.005 \mathrm{mmol})$ and TEMPO $(0.01 \mathrm{mmol})$ were dissolved in $10 \mathrm{~mL}$ of ethanol in a $25 \mathrm{~mL}$ glass vessel equipped with a $\mathrm{O}_{2}$ balloon. The solution was stirred at $60{ }^{\circ} \mathrm{C}$ under irradiated with $10 \mathrm{~W}$ blue LEDs $(\lambda=450-470 \mathrm{~nm})$ for $18 \mathrm{~h}$. After that, the reaction mixtures were analyzed by HRMS (see Figure S64).

\section{S5. For $\mathrm{H}_{2} \mathrm{O}_{2}$ Formation Experiment.}

The formation of $\mathrm{H}_{2} \mathrm{O}_{2}$ was monitored by UV-vis of tri-iodide $\left(\mathrm{I}_{3}^{-}\right)$in EtOH solution. When 3 eq. KI was added the standard reaction solution in $2 \mathrm{~h}$, the $\mathrm{I}^{-}$can be oxidized to $\mathrm{I}_{2}$ by $\mathrm{H}_{2} \mathrm{O}_{2}$ subsequently. The $\mathrm{I}_{2}$ further react with $\mathrm{I}^{-}$to form $\mathrm{I}_{3}{ }^{-}$, which shows two characteristic peaks at ca. 292 and $351 \mathrm{~nm}$ in UV-vis spectra (see Figure S65). 


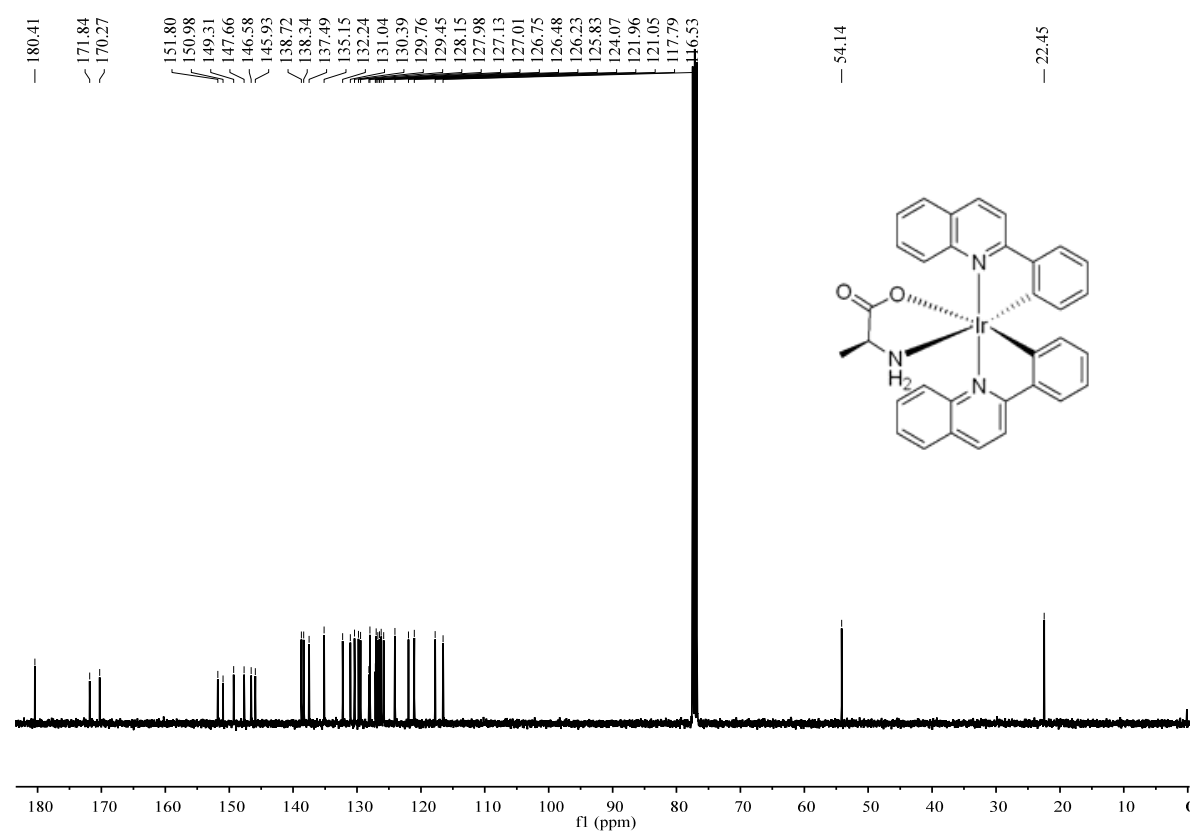

Figure S1. The ${ }^{13} \mathrm{C}$ NMR spectrum of $\Delta-\left[\operatorname{Ir}(\mathrm{pq})_{2}(L\right.$-ala $\left.)\right]$ or $\Lambda$-[ $\operatorname{Ir}(\mathrm{pq})_{2}(D$-ala $\left.)\right]$ in $\mathrm{CDCl}_{3}$.
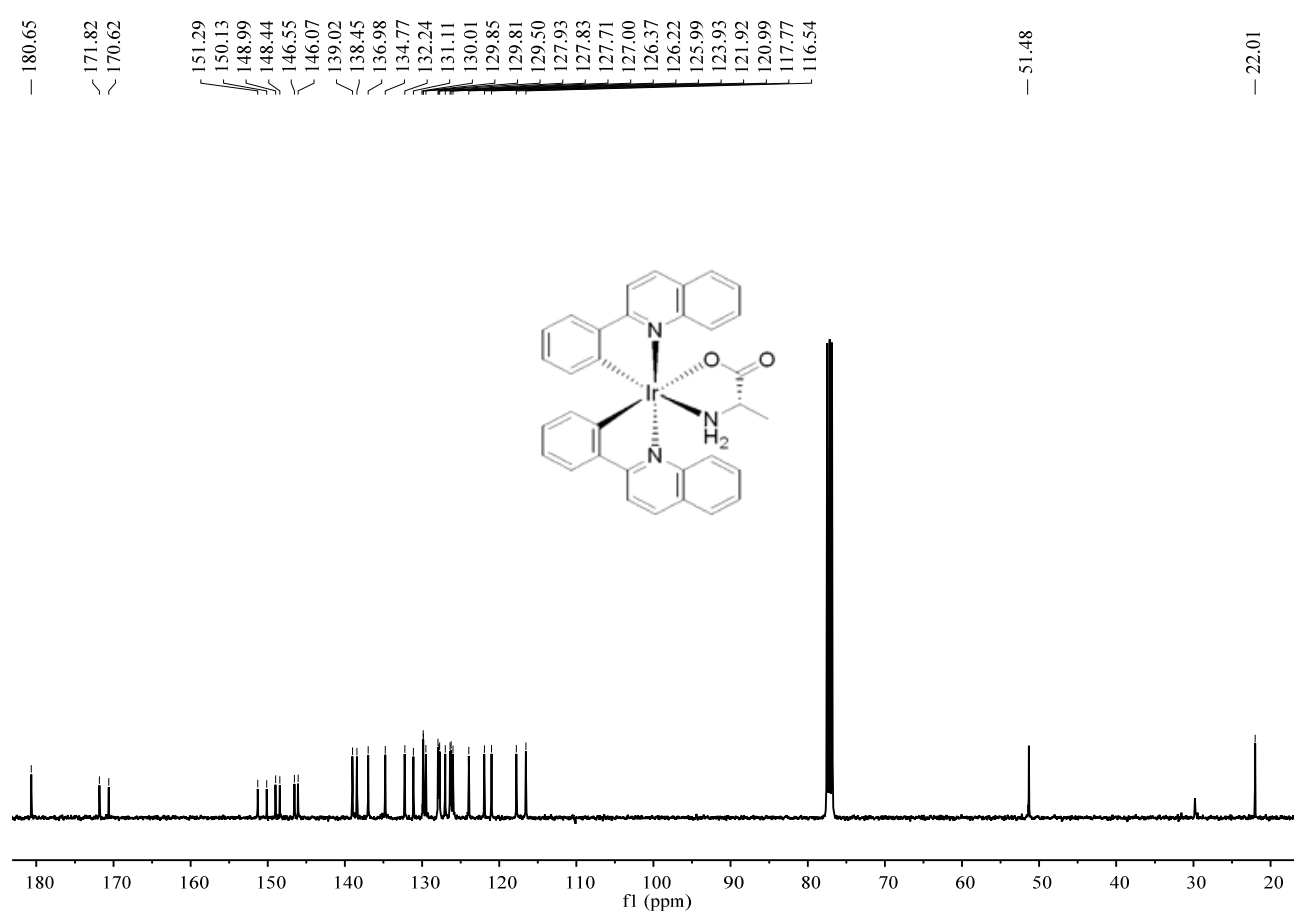

Figure S2. The ${ }^{13} \mathrm{C}$ NMR spectrum of $\Delta-\left[\operatorname{Ir}(\mathrm{pq})_{2}(D\right.$-ala $\left.)\right]$ or $\Lambda$-[ $\operatorname{Ir}(\mathrm{pq})_{2}(L$-ala $\left.)\right]$ in $\mathrm{CDCl}_{3}$. 


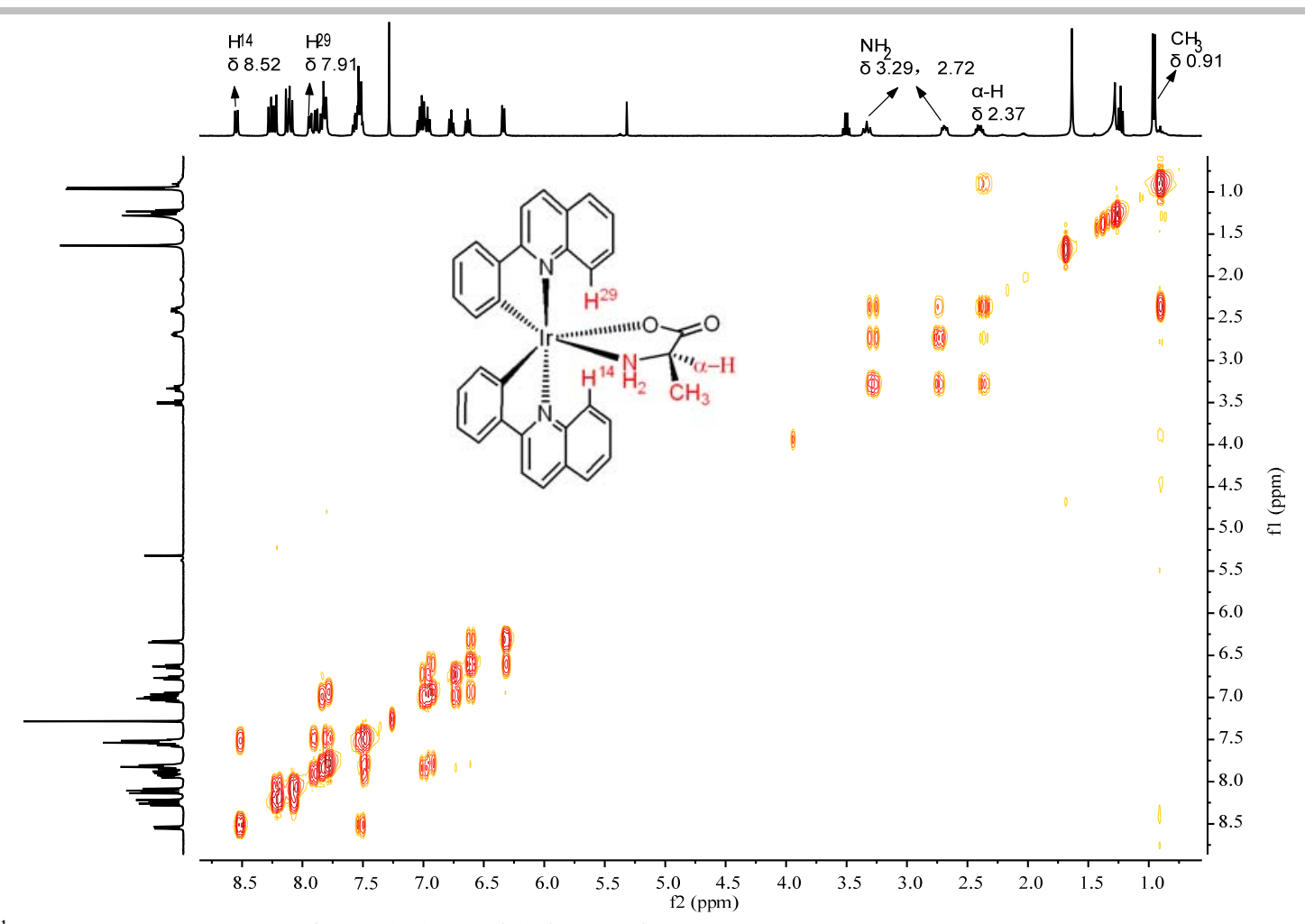

Figure S3. ${ }^{1} \mathrm{H}$ NMR-COSY spectra of $\Lambda$-[ $\operatorname{Ir}(\mathrm{pq})_{2}(D$-ala $\left.)\right]$ in $\mathrm{CDCl}_{3}$.

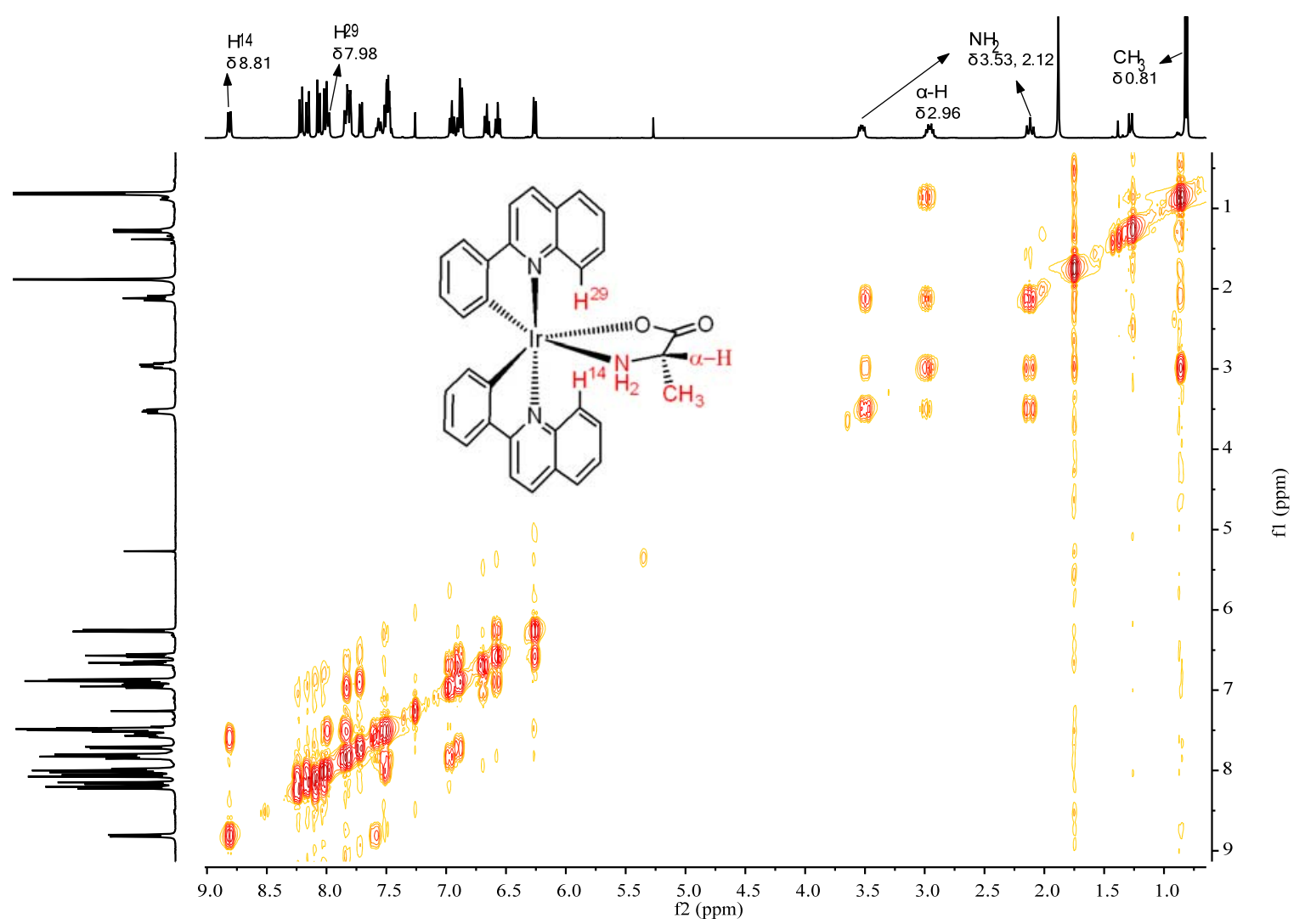

Figure S4. ${ }^{1} \mathrm{H}$ NMR-COSY spectra of $\Lambda$-[ $\operatorname{Ir}(\mathrm{pq})_{2}(L$-ala $\left.)\right]$ in $\mathrm{CDCl}_{3}$. 


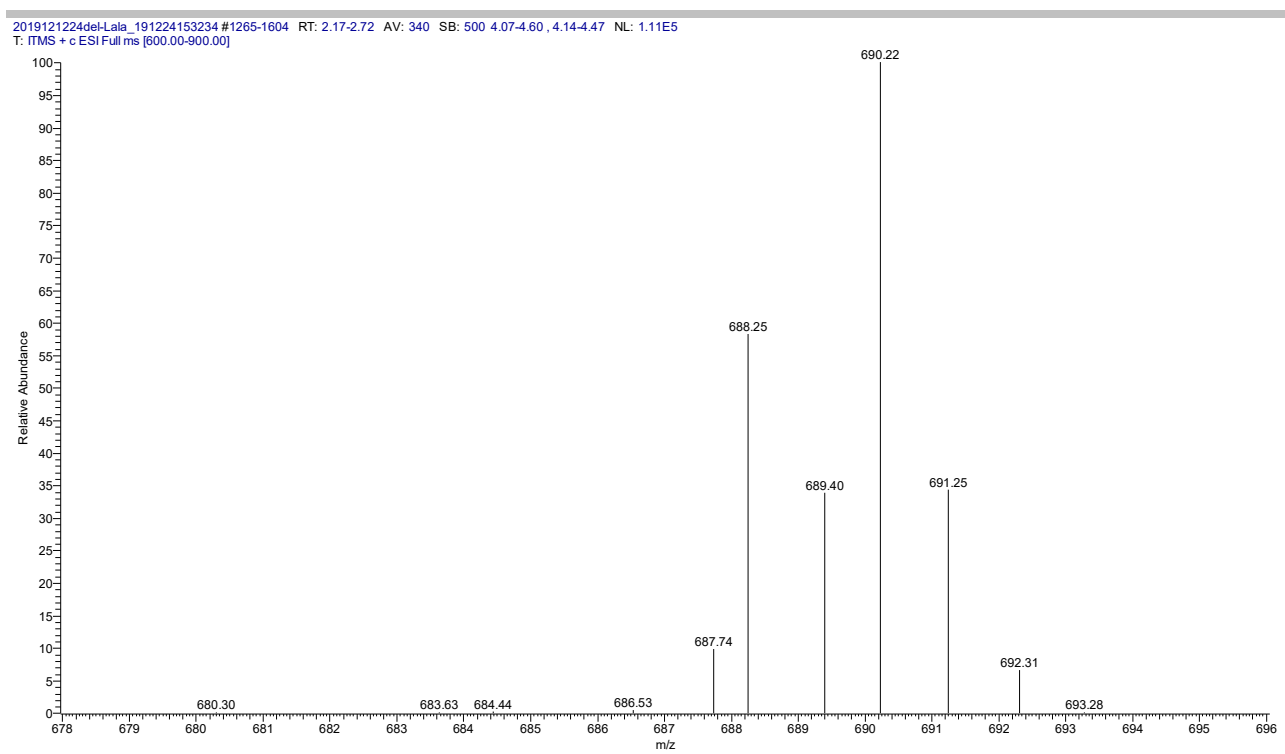

Figure S5. The MS spectrum of $\Lambda$-[Ir(pq $)_{2}(D$-ala $\left.)\right]\left([\mathrm{M}+\mathrm{H}]^{+}\right)$.
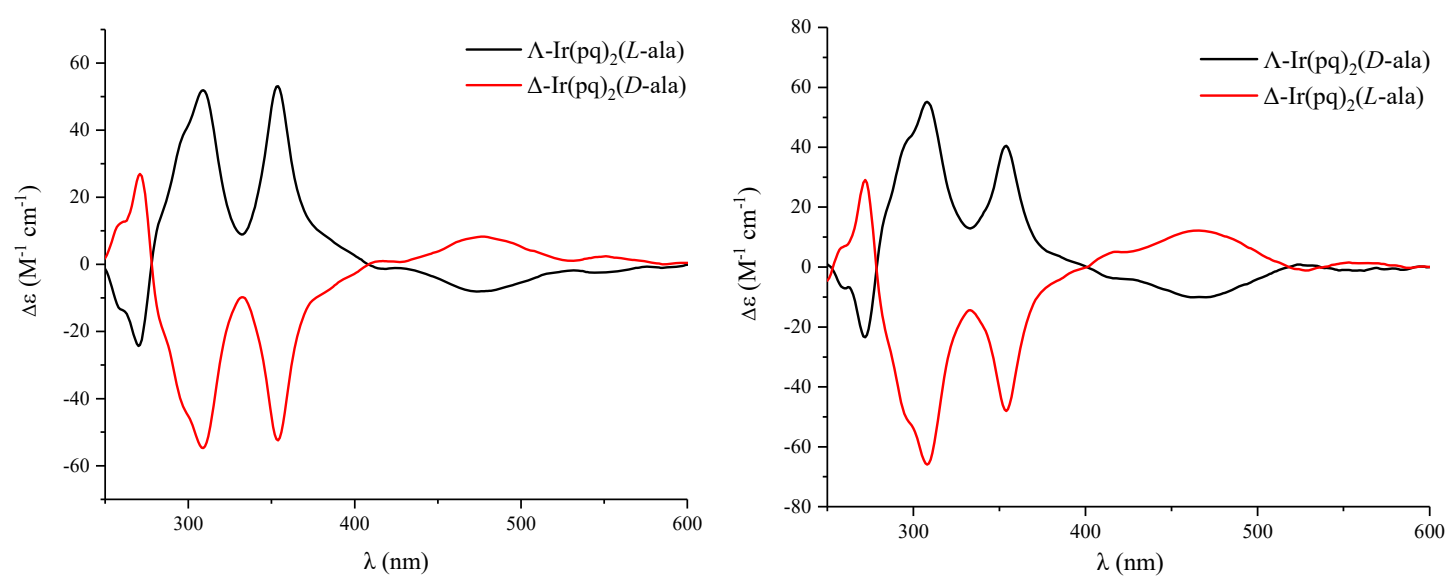

Figure S6. CD spectra of $\Lambda$-[Ir(pq $)_{2}(L$-ala $\left.)\right]$ and $\Delta-\left[\operatorname{Ir}(\mathrm{pq})_{2}(D\right.$-ala $\left.)\right]($ left $)$ and $\Lambda$-[Ir(pq $)_{2}(D$-ala $\left.)\right]$ and $\Delta$-[Ir(pq $)_{2}(L$-ala $\left.)\right]($ right $)$ in $\mathrm{MeOH}(50 \mu \mathrm{M})$.
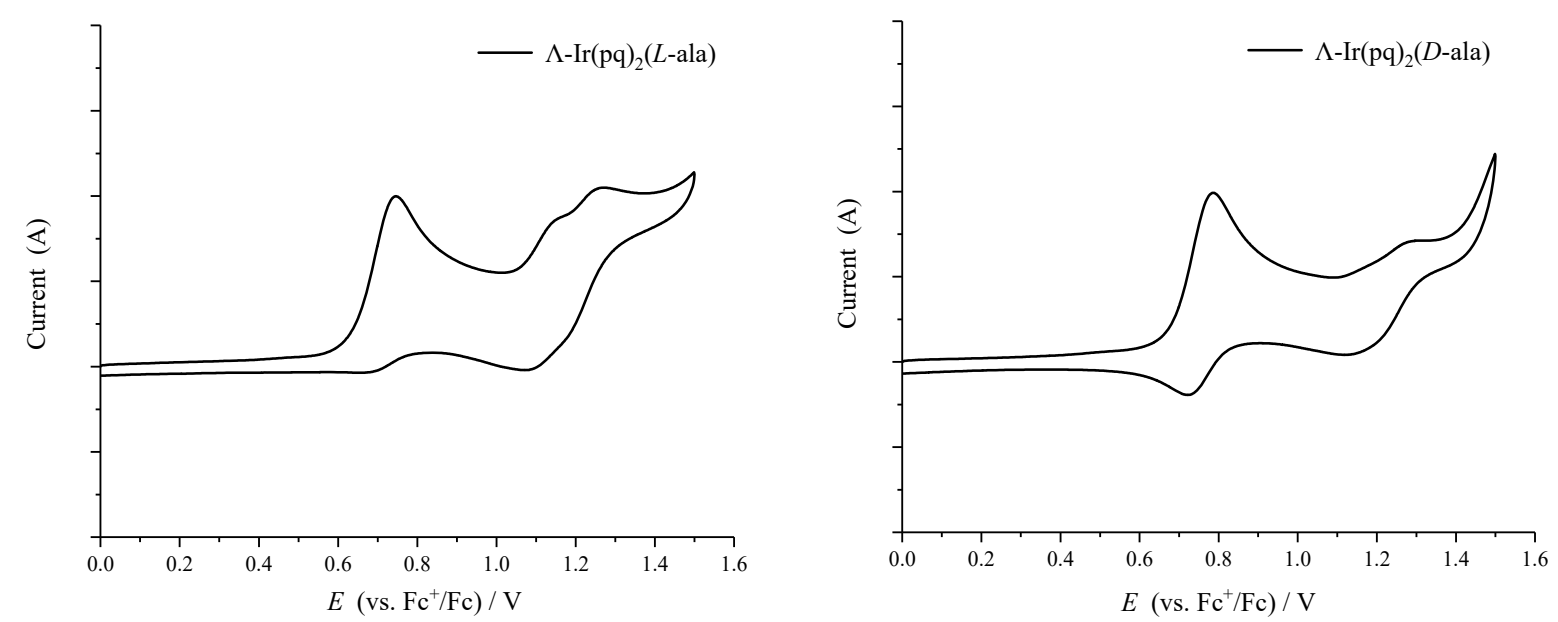

Figure S7. Cyclic voltammograms of $\Lambda$-[ $\operatorname{Ir}(\mathrm{pq})_{2}(L$-ala $\left.)\right]($ left $)$ and $\Lambda$-[ $\operatorname{Ir}(\mathrm{pq})_{2}(D$-ala $\left.)\right]$ (right) in $\mathrm{MeCN}$ solution $(0.001 \mathrm{~mol} / \mathrm{L})$ at a scan rate of $100 \mathrm{mV} / \mathrm{s}$. 

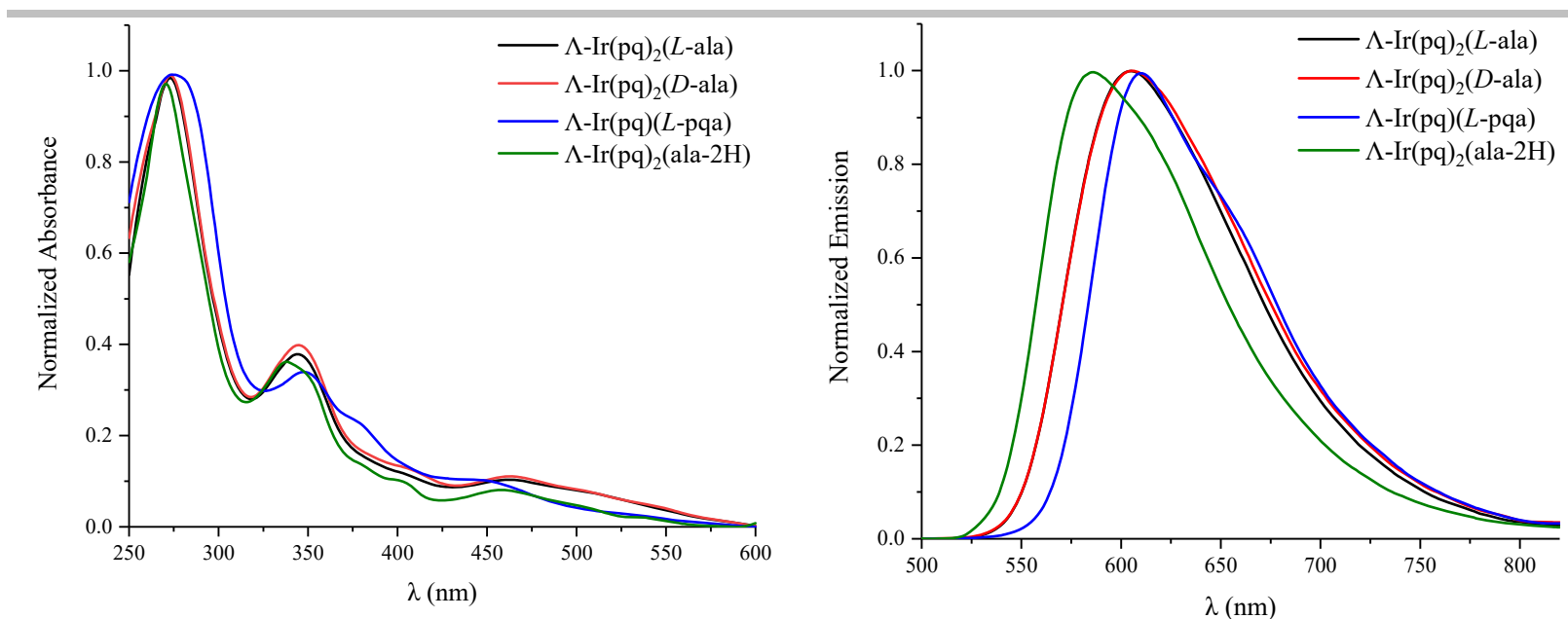

Figure S8. Normalized absorption and emission spectra of $\operatorname{Ir}(\mathrm{III})$ complexes in air-saturated $\mathrm{MeOH}$ solution $\left(10^{-5} \mathrm{M}\right)$ at room temperature.
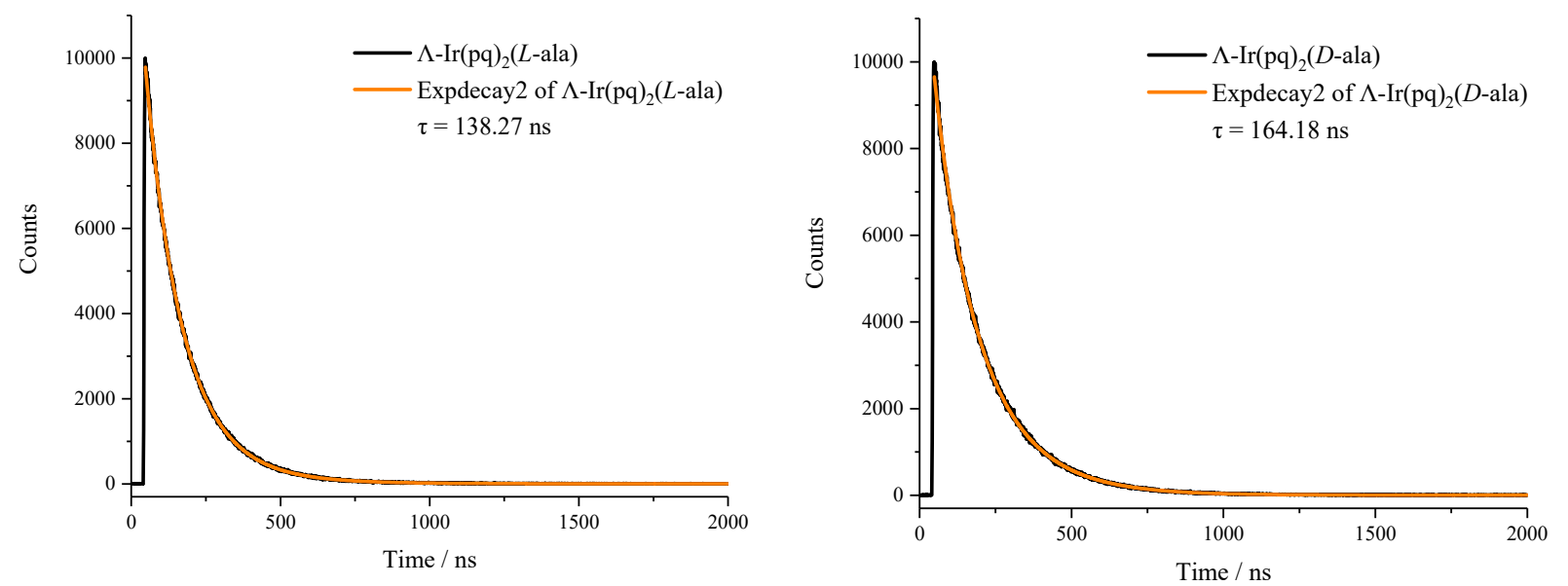

Figure S9. Lifetime decay curves and corresponding fitting curves for $\Lambda$-[Ir(pq) $)_{2}(L$-ala $\left.)\right]($ left $)$ and $\Lambda$ - $\left[\operatorname{Ir}(\mathrm{pq})_{2}(D\right.$-ala $\left.)\right]($ right $)$ in airsaturated $\mathrm{MeOH}$ solution $\left(10^{-5} \mathrm{M}\right)$ at room temperature.
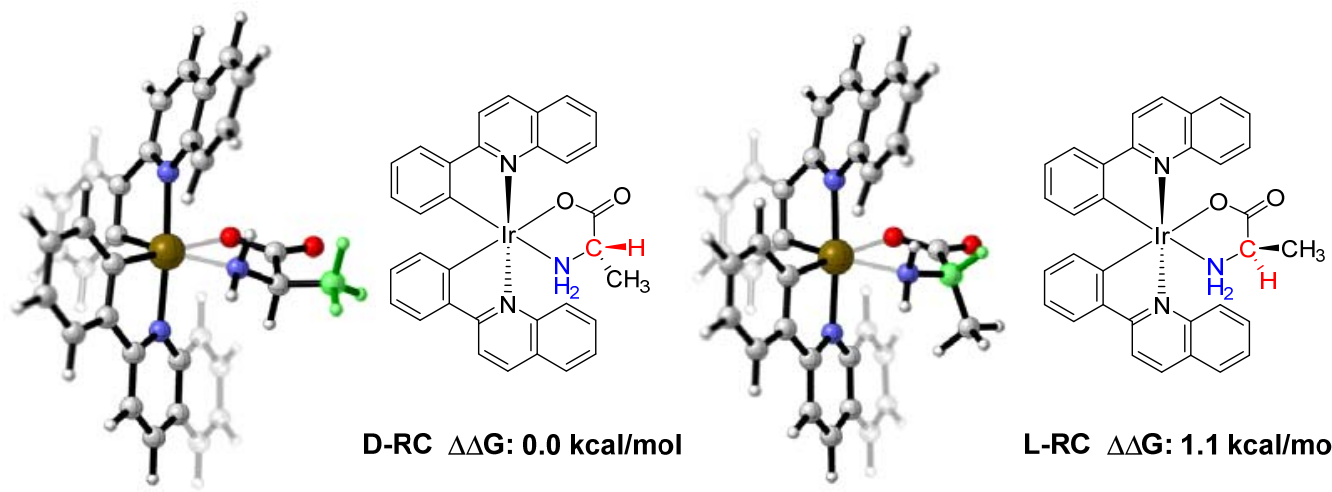

L-RC $\Delta \Delta G: 1.1 \mathrm{kcal} / \mathrm{mol}$

Figure S10. The Gibbs free energy for $\Lambda$ - $\left[\operatorname{Ir}(\mathrm{pq})_{2}(D\right.$-ala $\left.)\right]$ and $\Lambda$-[Ir(pq $)_{2}(L$-ala $\left.)\right]$ diastereomers at at wB97XD/def2-TZVP, SMD $(\mathrm{EtOH}) / / \mathrm{wB} 97 \mathrm{XD} / \mathrm{def} 2-\mathrm{SVP}$ level of theory. 
E
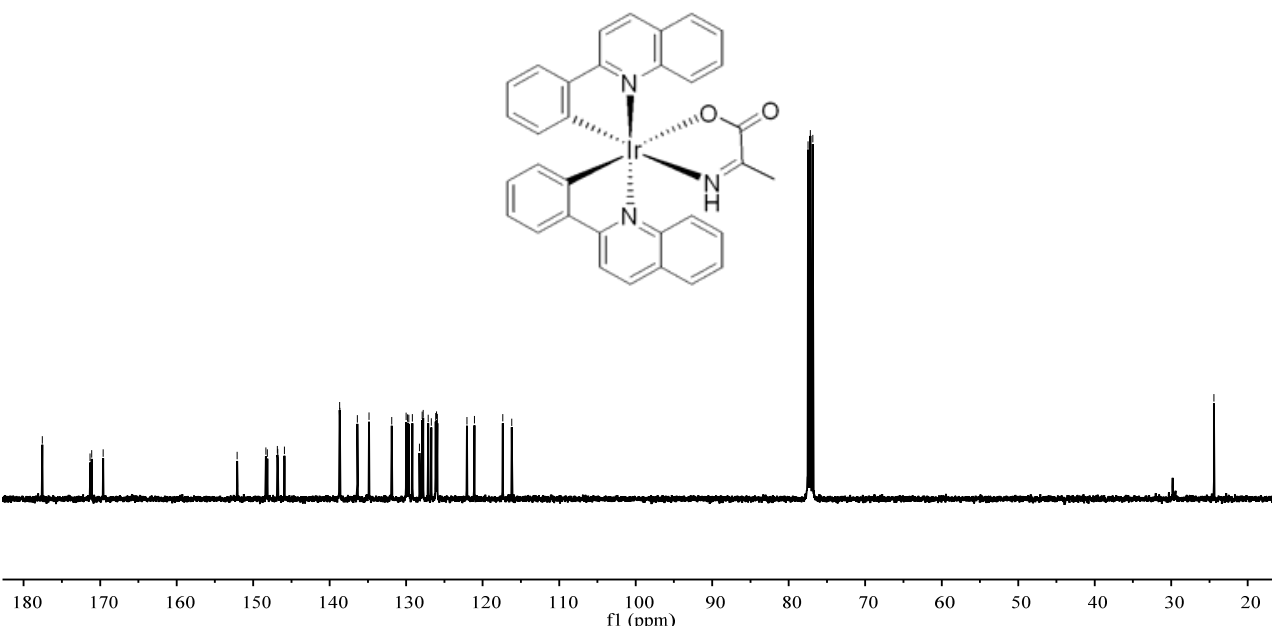

Figure S11. The ${ }^{13} \mathrm{C}$ NMR spectrum of $\Lambda$ - $\left[\mathrm{Ir}(\mathrm{pq})_{2}(\right.$ ala-2 $\left.\mathrm{H})\right]$ in $\mathrm{CDCl}_{3}$.

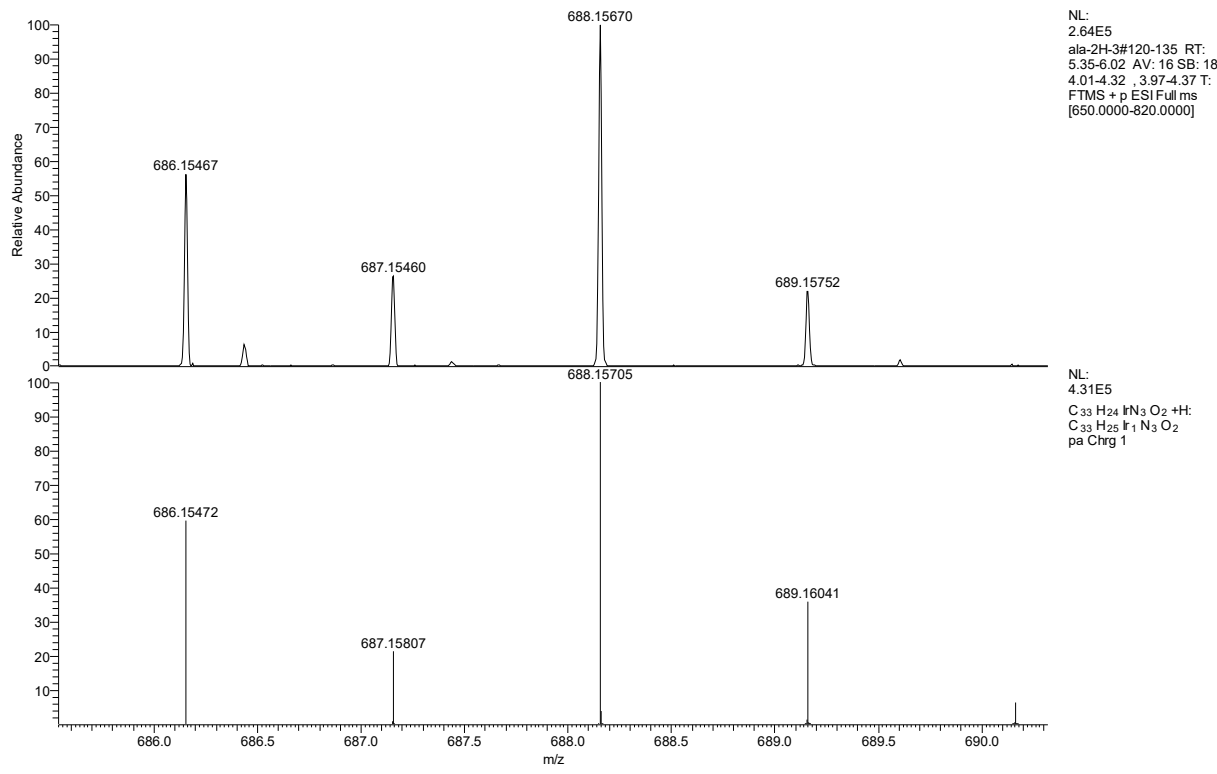

Figure S12. Comparison of HRMS of $\Lambda$-[Ir(pq) $\left(\right.$ ala-2H)] at m/z $688.15670\left([\mathrm{M}+\mathrm{H}]^{+}\right)($up $)$and calculated for $\Lambda$-[ $\left.\operatorname{Ir}(\mathrm{pq})_{2}(\mathrm{ala}-2 \mathrm{H})\right]$ $\left(\left[\mathrm{C}_{33} \mathrm{H}_{24} \mathrm{IrN}_{3} \mathrm{O}_{2}+\mathrm{H}\right]^{+}, 688.15705\right.$, down). 

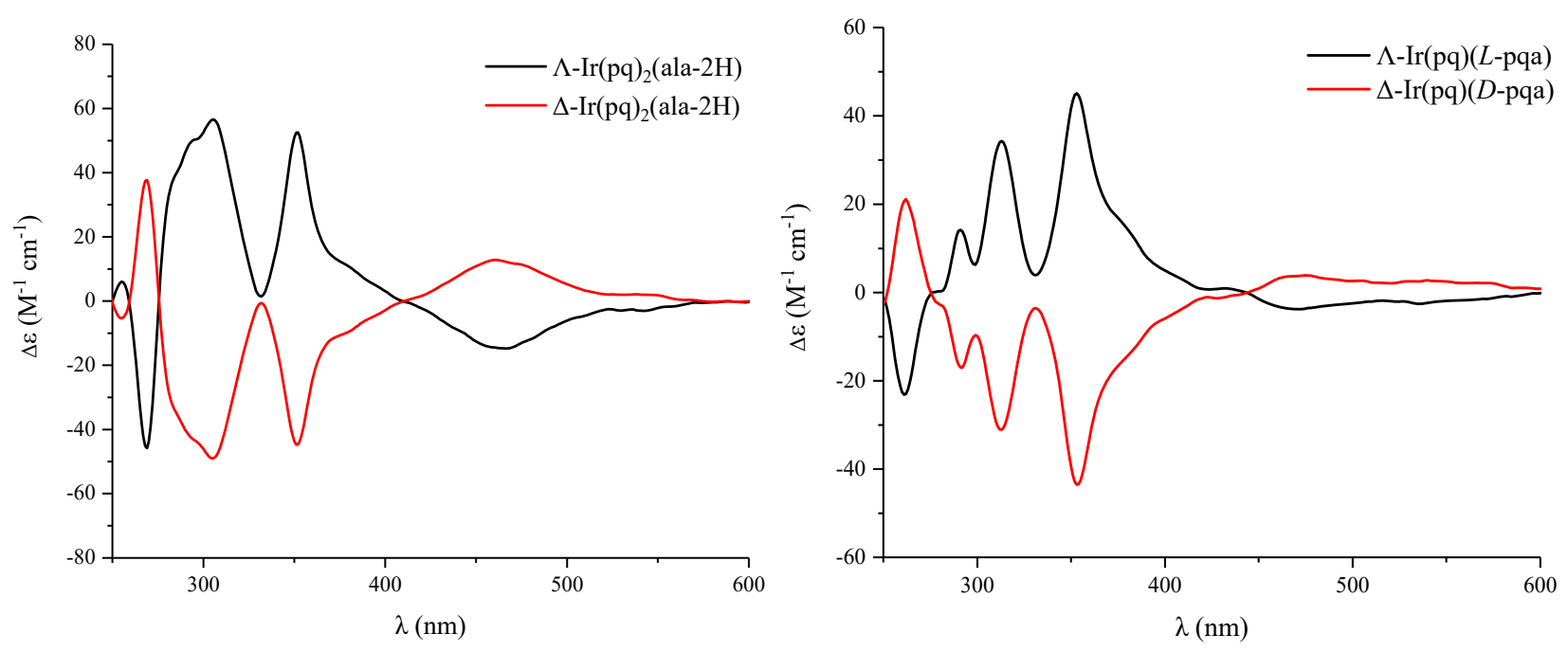

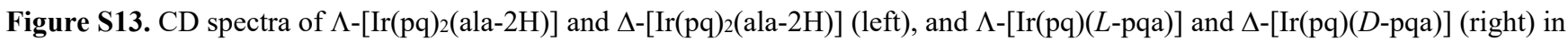
$\mathrm{MeOH}(50 \mu \mathrm{M})$.
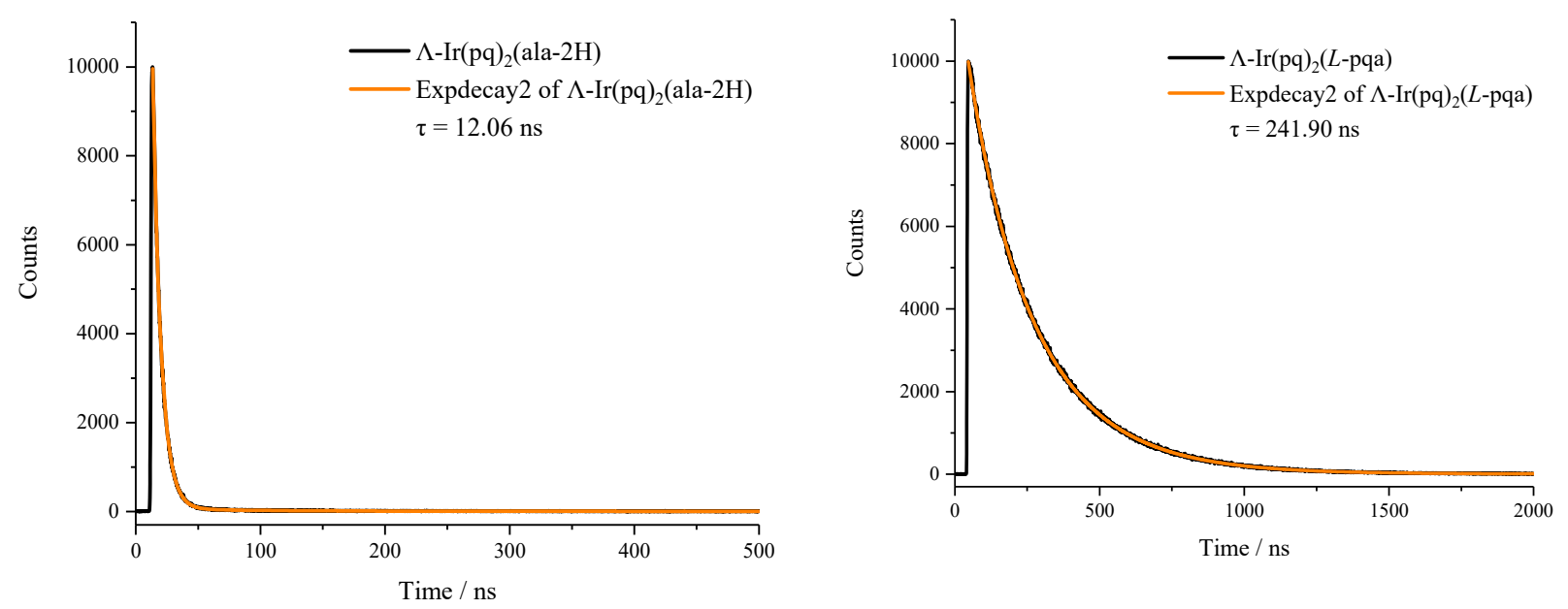

Figure S14. Lifetime decay curves and corresponding fitting curves for $\Lambda$-[ $\operatorname{Ir}(\mathrm{pq})_{2}(L$-ala) $]$ (left) and $\Lambda$-[ $\operatorname{Ir}(\mathrm{pq})(L$-pqa) $]$ (right) in airsaturated $\mathrm{MeOH}$ solution $\left(10^{-5} \mathrm{M}\right)$ at room temperature. 


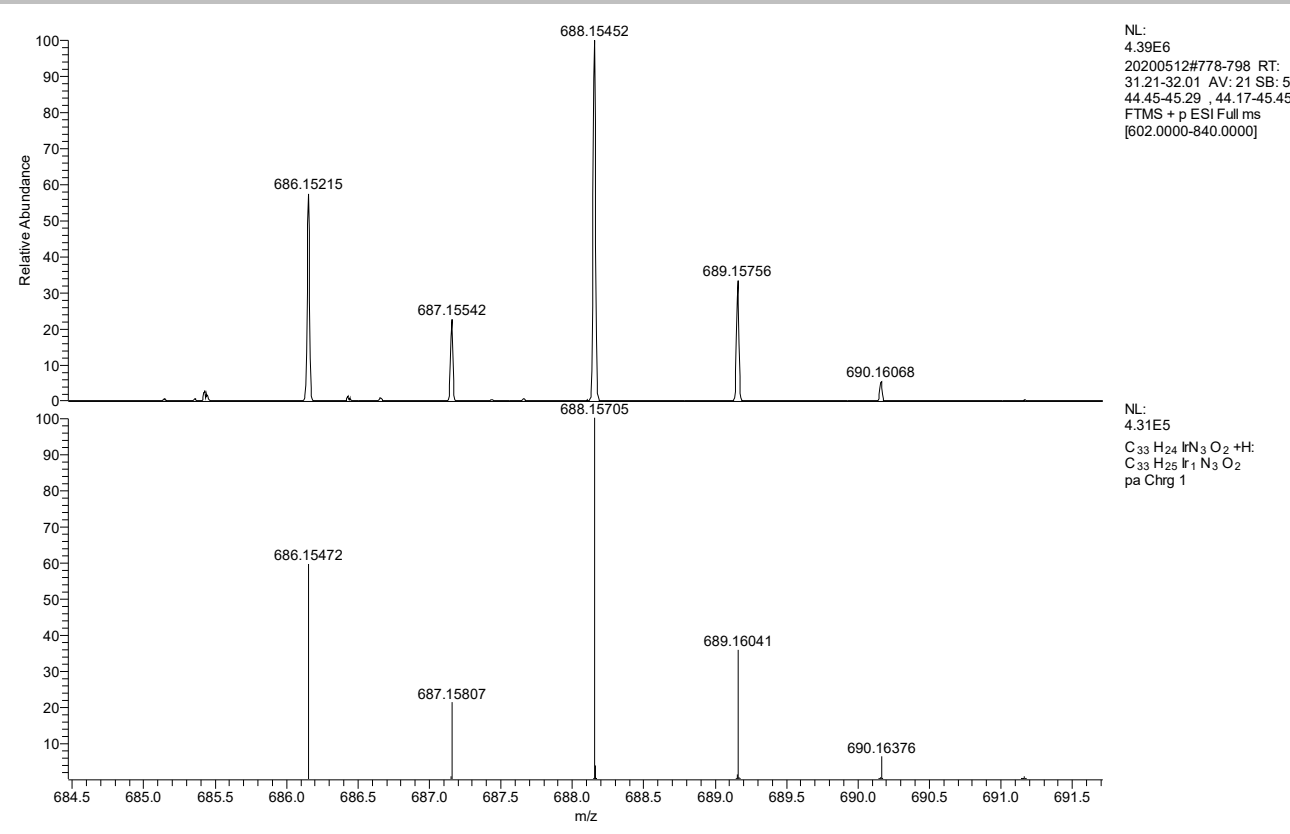

Figure S15. Comparison of HRMS of $\Lambda$-[Ir(pq) $(L-\mathrm{pqa})]$ at m/z $688.15452\left([\mathrm{M}+\mathrm{H}]^{+}\right)$(up) and calculated for $\Lambda$-[ $\left.\operatorname{Ir}(\mathrm{pq})(L-\mathrm{pqa})\right]$ $\left(\left[\mathrm{C}_{33} \mathrm{H}_{24} \mathrm{IrN}_{3} \mathrm{O}_{2}+\mathrm{H}\right]^{+}, 688.15705\right.$, down).
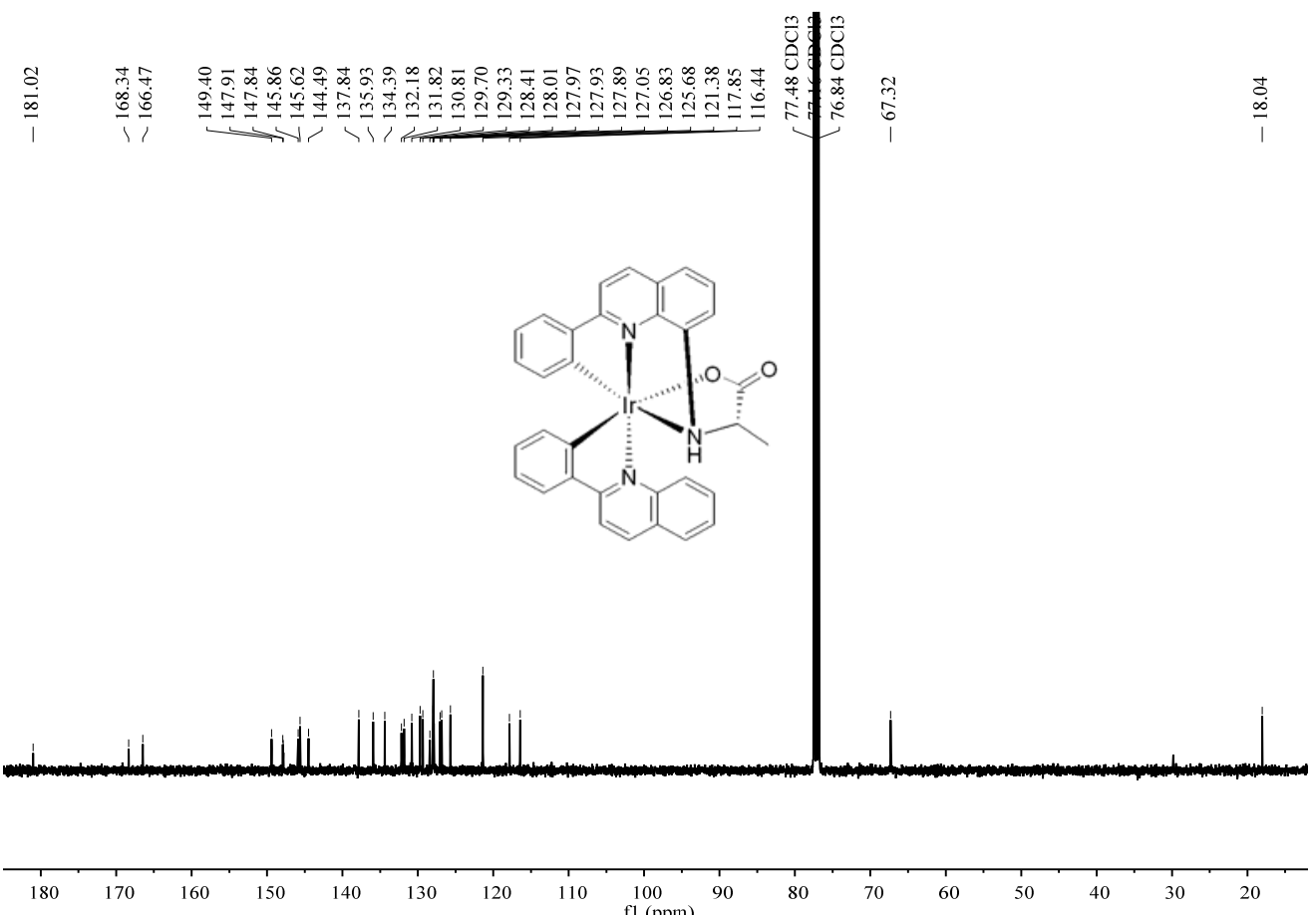

Figure S16. The ${ }^{13} \mathrm{C}$ NMR spectrum of $\Lambda-[\operatorname{Ir}(\mathrm{pq})(L-\mathrm{pqa})]$ in $\mathrm{CDCl}_{3}$. 


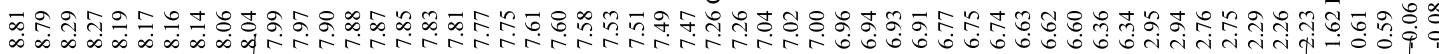
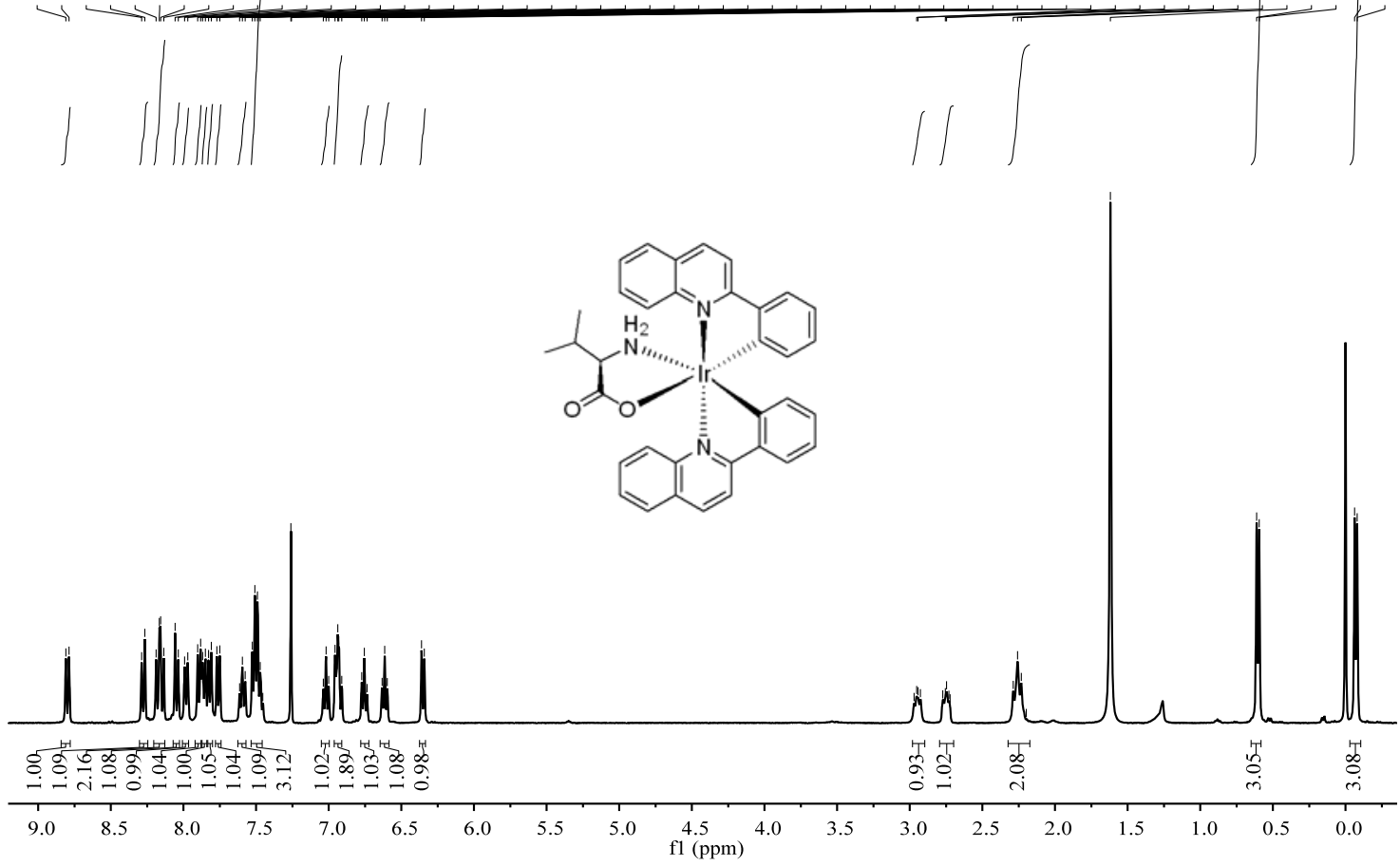

Figure S17. The ${ }^{1} \mathrm{H}$ NMR spectrum of $\Delta-\left[\operatorname{Ir}(\mathrm{pq})_{2}(D\right.$-val $\left.)\right]$ in $\mathrm{CDCl}_{3}$.
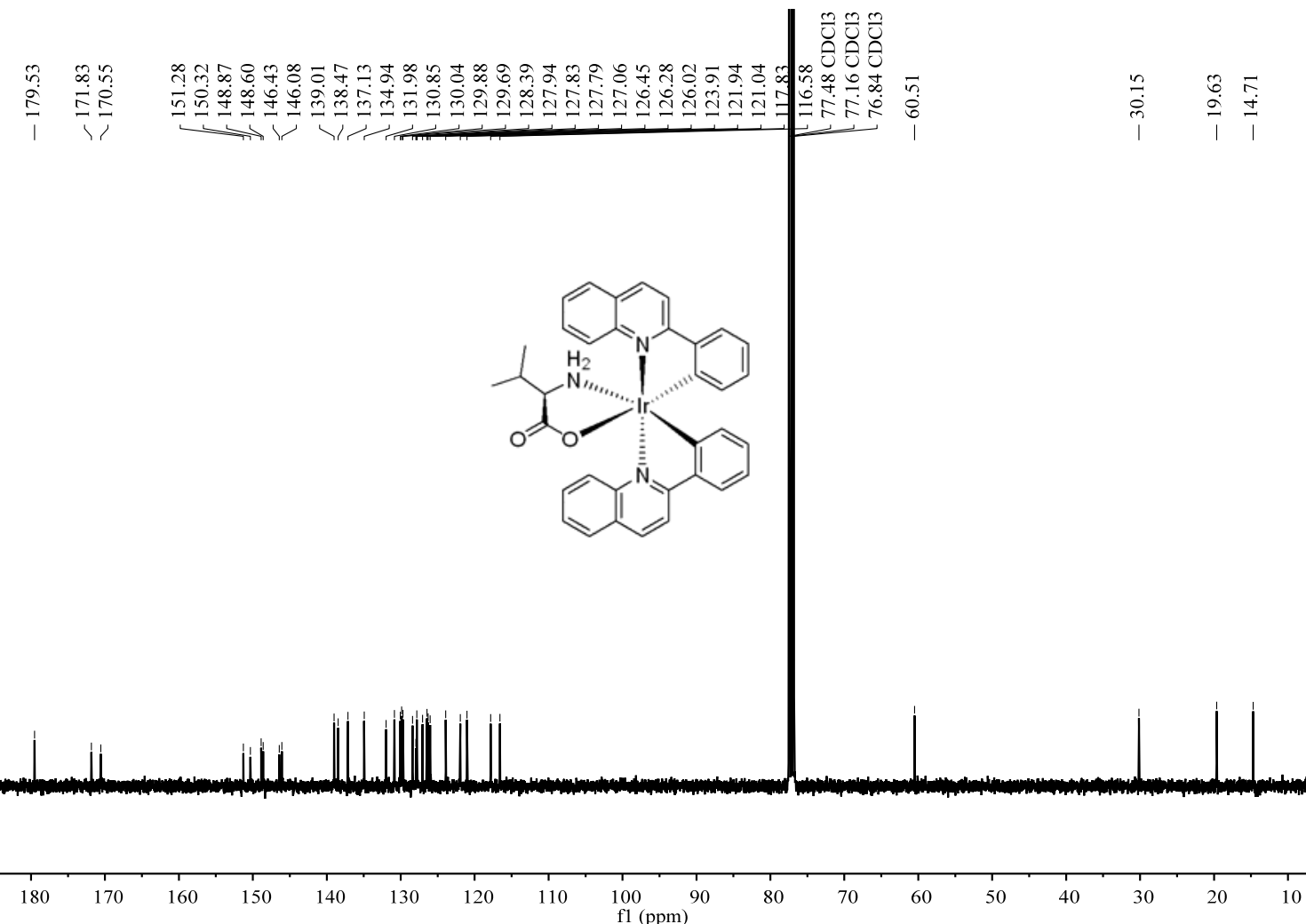

Figure S18. The ${ }^{13} \mathrm{C}$ NMR spectrum of $\Delta-\left[\operatorname{Ir}(\mathrm{pq})_{2}(D\right.$-val $\left.)\right]$ in $\mathrm{CDCl}_{3}$. 


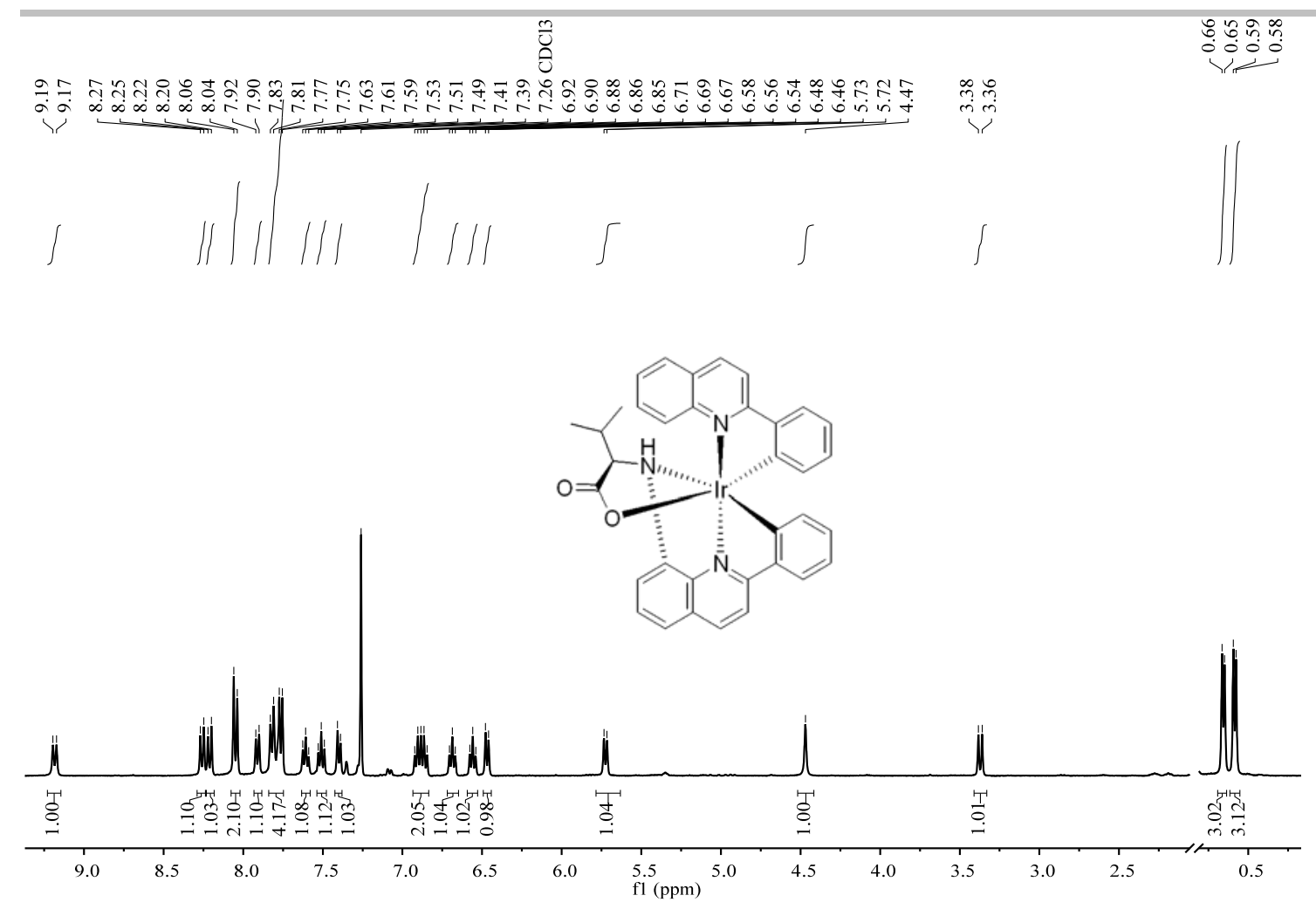

Figure S19. The ${ }^{1} \mathrm{H}$ NMR spectrum of $\Delta$-[ $\left.\operatorname{Ir}(\mathrm{pq})(D-\mathrm{pqv})\right]$ in $\mathrm{CDCl}_{3}$.

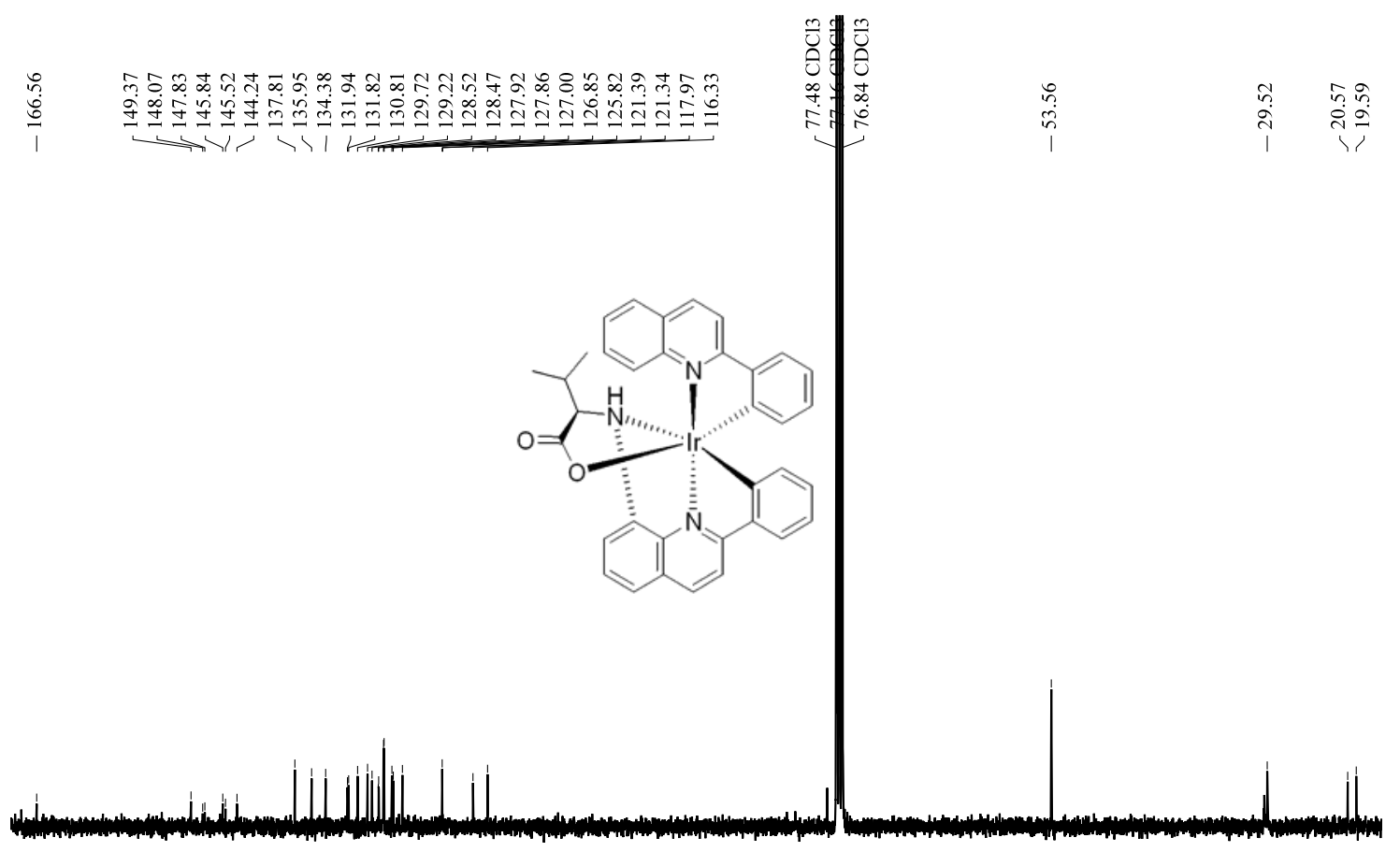

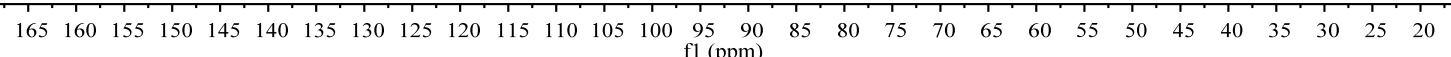

Figure S20. The ${ }^{13} \mathrm{C}$ NMR spectrum of $\Delta-[\operatorname{Ir}(\mathrm{pq})(D-\mathrm{pqv})]$ in $\mathrm{CDCl}_{3}$. 


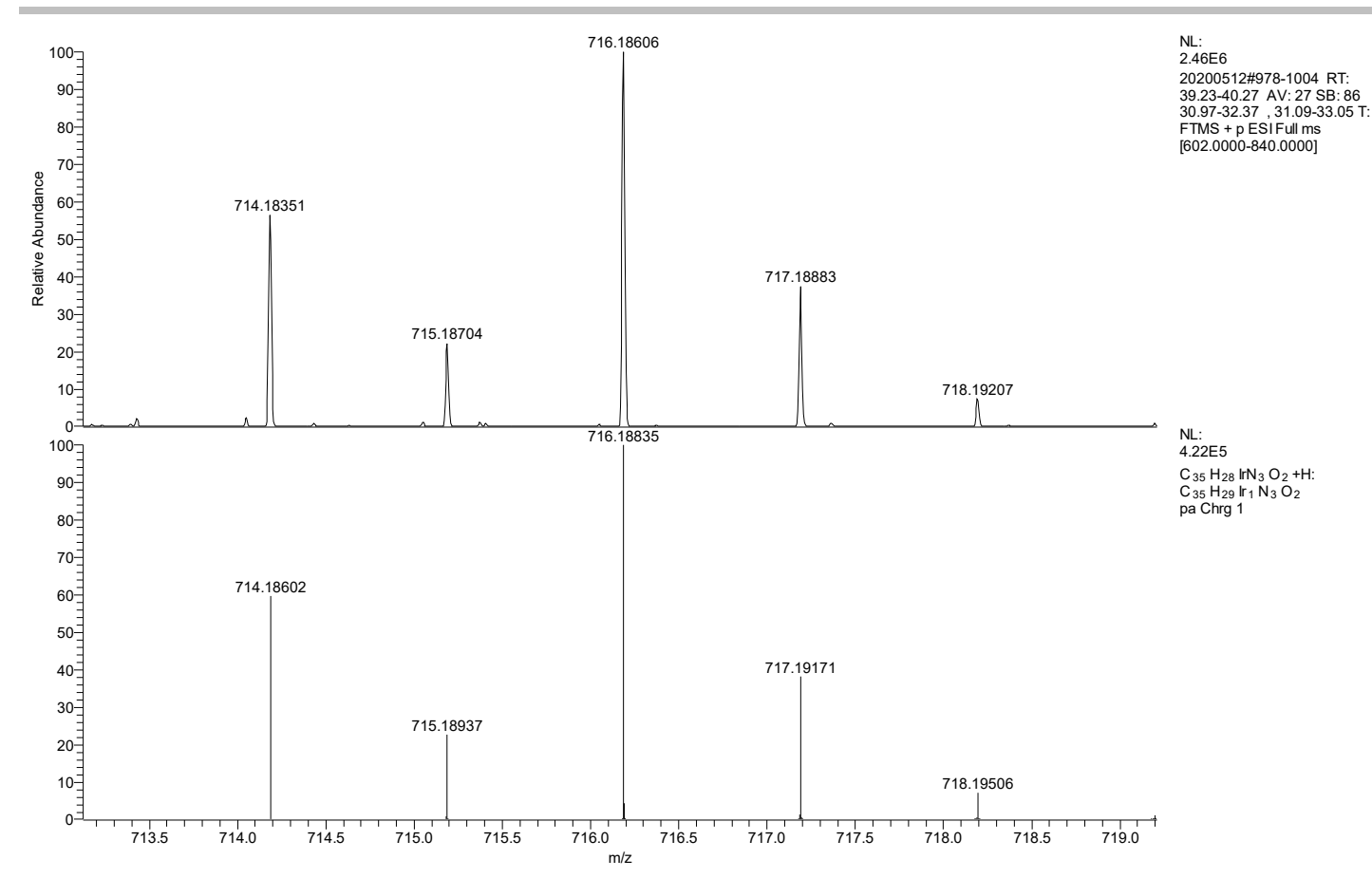

Figure S21. Comparison of HRMS of $\Delta$-[Ir(pq)(D-pqv)] at m/z $716.18606\left([\mathrm{M}+\mathrm{H}]^{+}\right)(\mathrm{up})$ and calculated for $\Delta$-[Ir(pq)(D-pqv)] $\left(\left[\mathrm{C}_{35} \mathrm{H}_{28} \mathrm{IrN}_{3} \mathrm{O}_{2}+\mathrm{H}\right]^{+}, 716.18835\right.$, down).

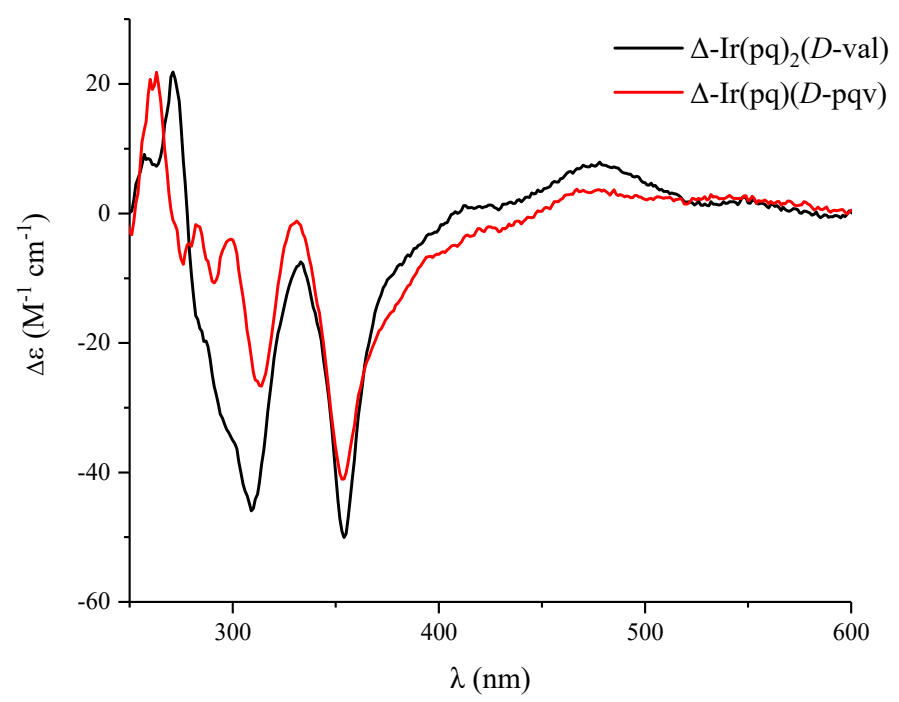

Figure S22. CD spectra of $\Delta$-[Ir(pq $\left.)_{2}(D-\mathrm{val})\right]$ and $\Delta-[\operatorname{Ir}(\mathrm{pq})(D-\mathrm{pqv})]$ in $\mathrm{MeOH}(50 \mu \mathrm{M})$. 

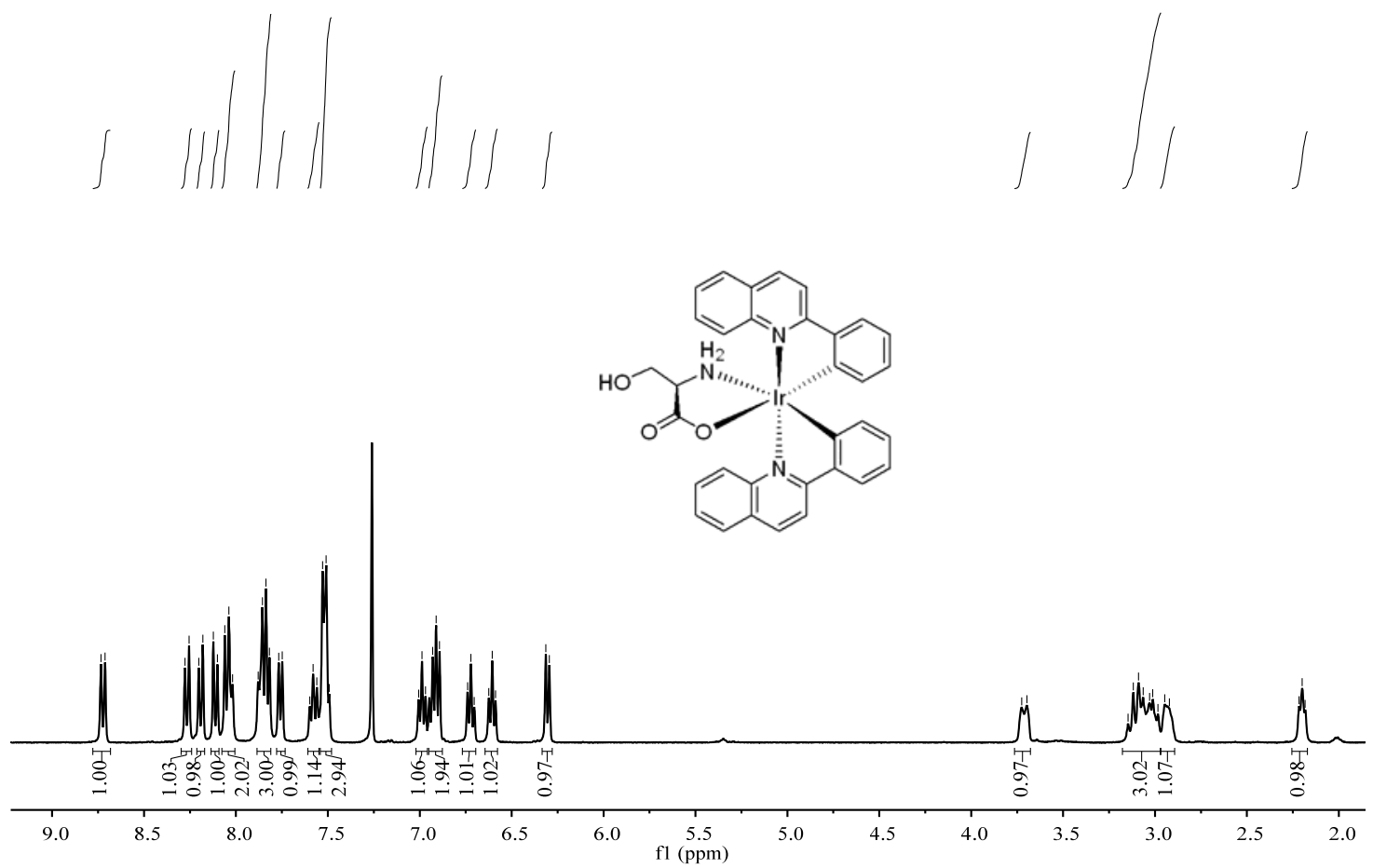

Figure S23. The ${ }^{1} \mathrm{H}$ NMR spectrum of $\Delta-\left[\operatorname{Ir}(\mathrm{pq})_{2}(D\right.$-ser $\left.)\right]$ in $\mathrm{CDCl}_{3}$.

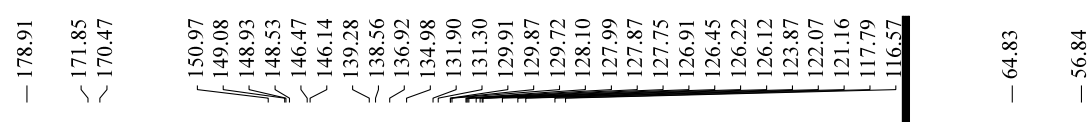
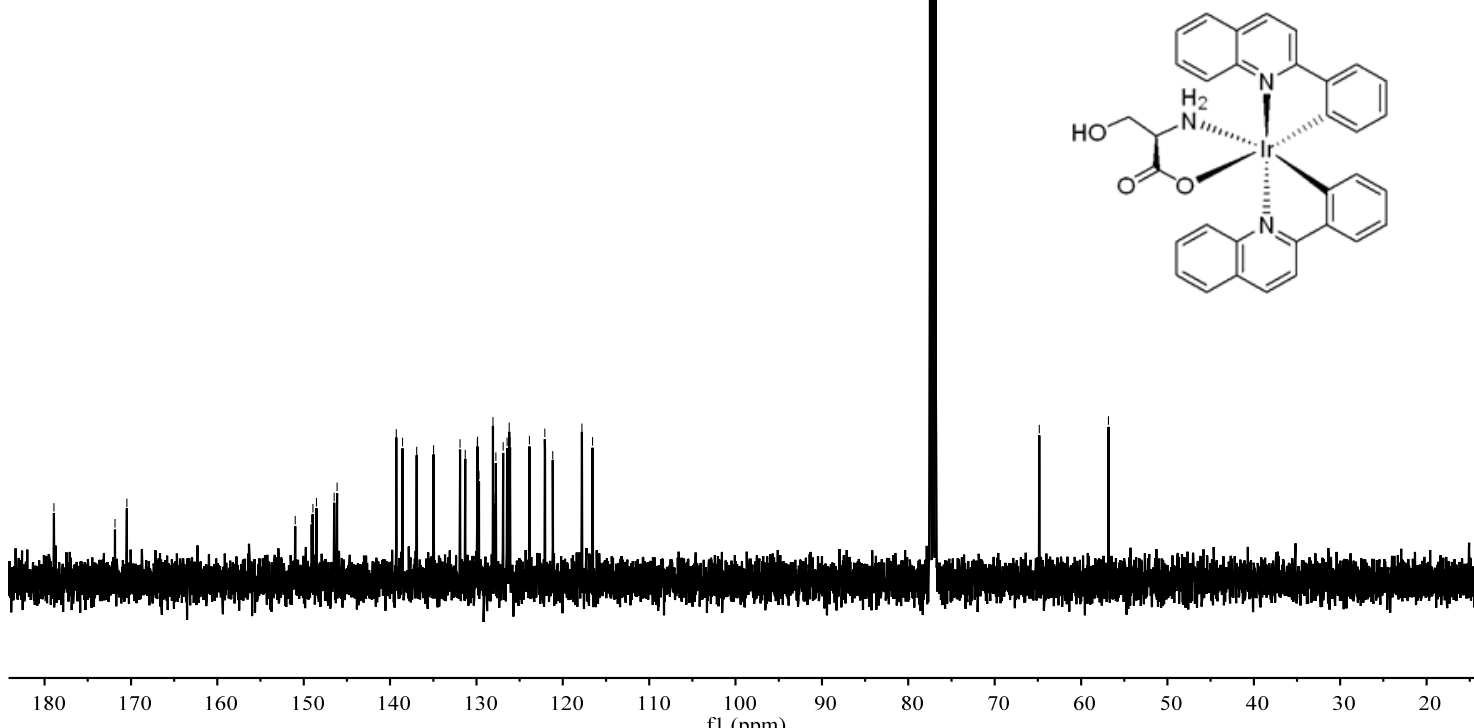

Figure S24. The ${ }^{13} \mathrm{C}$ NMR spectrum of $\Delta-\left[\operatorname{Ir}(\mathrm{pq})_{2}(D\right.$-ser $\left.)\right]$ in $\mathrm{CDCl}_{3}$. 


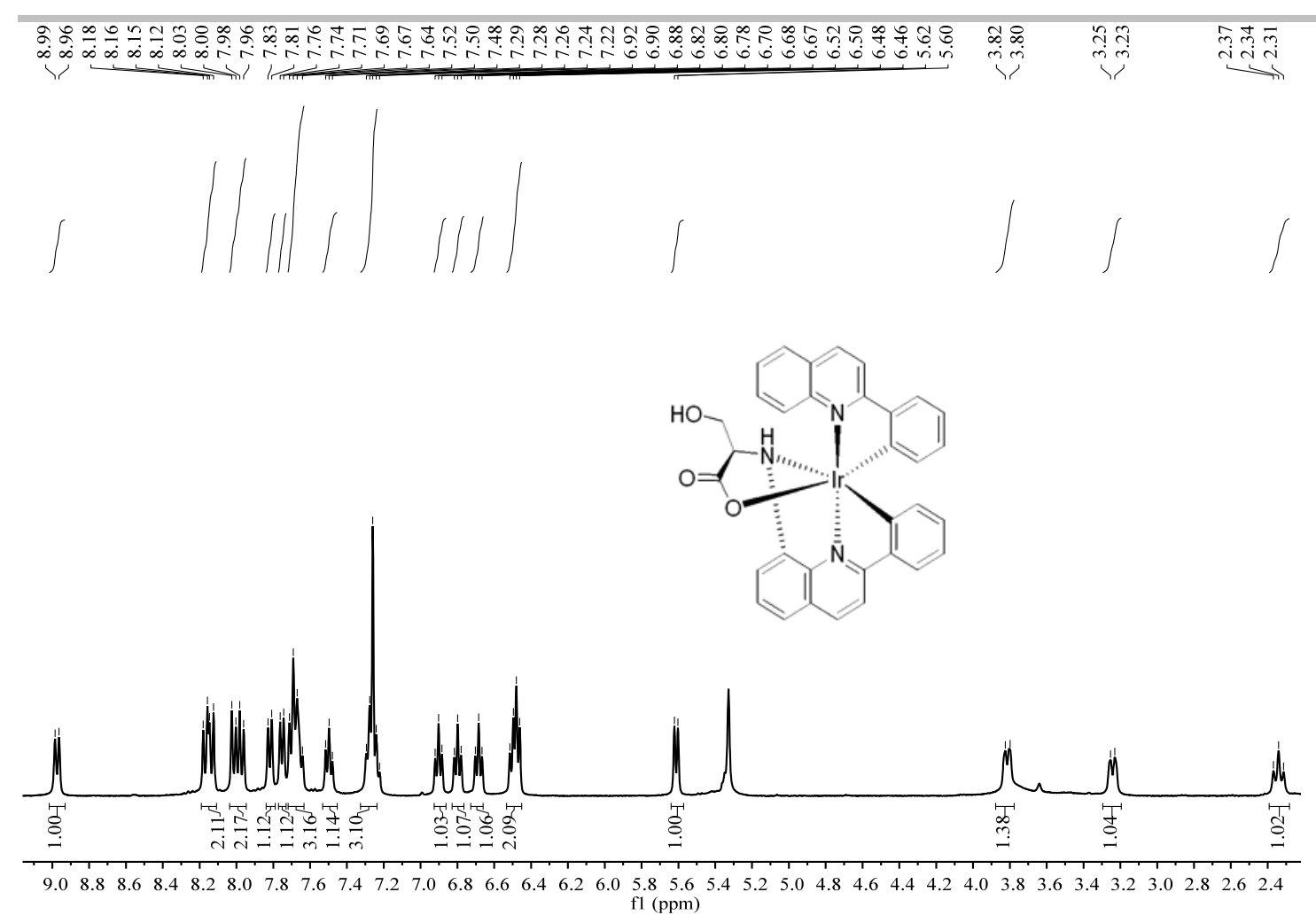

Figure S25. The ${ }^{1} \mathrm{H}$ NMR spectrum of $\Delta-[\operatorname{Ir}(\mathrm{pq})(D-\mathrm{pqs})]$ in $\mathrm{CDCl}_{3}$.

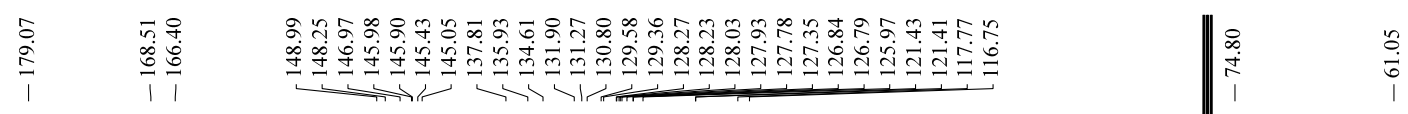

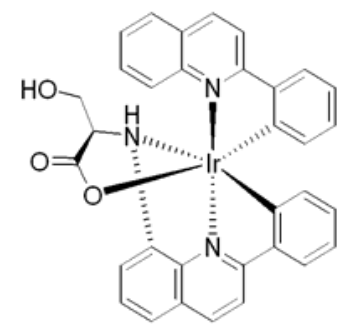

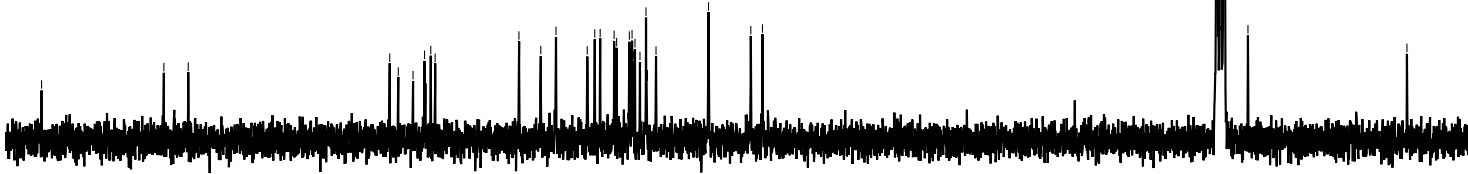

$\begin{array}{lllllllllllllllllllllllll}180 & 175 & 170 & 165 & 160 & 155 & 150 & 145 & 140 & 135 & 130 & 125 & \begin{array}{l}120 \\ \mathrm{fl}(\mathrm{ppm})\end{array} & 115 & 110 & 105 & 100 & 95 & 90 & 85 & 80 & 75 & 70 & 65 & 60\end{array}$

Figure S26. The ${ }^{13} \mathrm{C}$ NMR spectrum of $\Delta-[\operatorname{Ir}(\mathrm{pq})(D-\mathrm{pqs})]$ in $\mathrm{CDCl}_{3}$. 


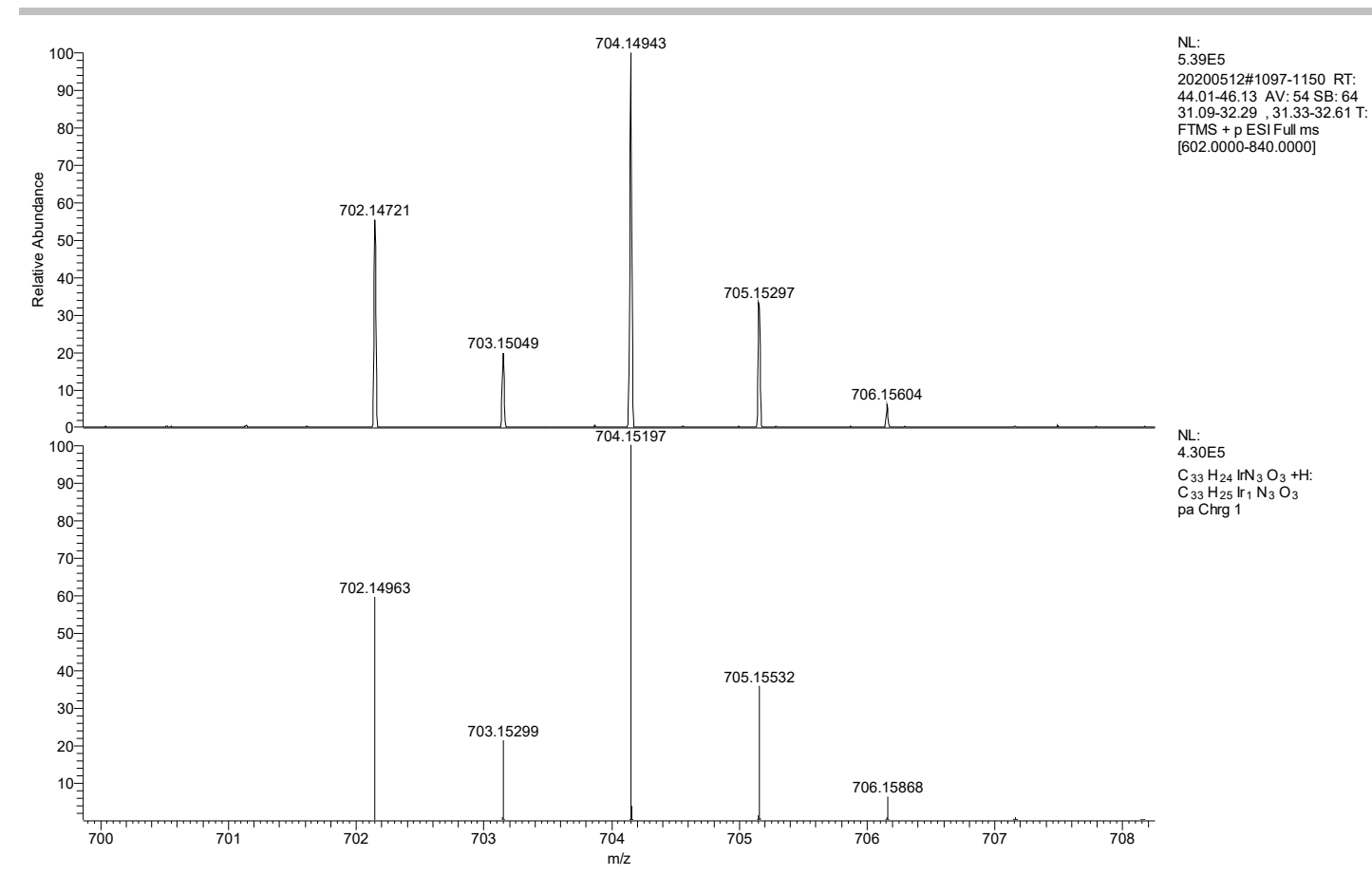

Figure S27. Comparison of HRMS of $\Delta$-[Ir(pq)(D-pqs)] at m/z $704.1943\left([\mathrm{M}+\mathrm{H}]^{+}\right)($up) and calculated for $\Delta$-[Ir(pq)(D-pqs)] $\left(\left[\mathrm{C}_{33} \mathrm{H}_{24} \mathrm{IrN}_{3} \mathrm{O}_{3}+\mathrm{H}\right]^{+}, 704.15197\right.$, down).

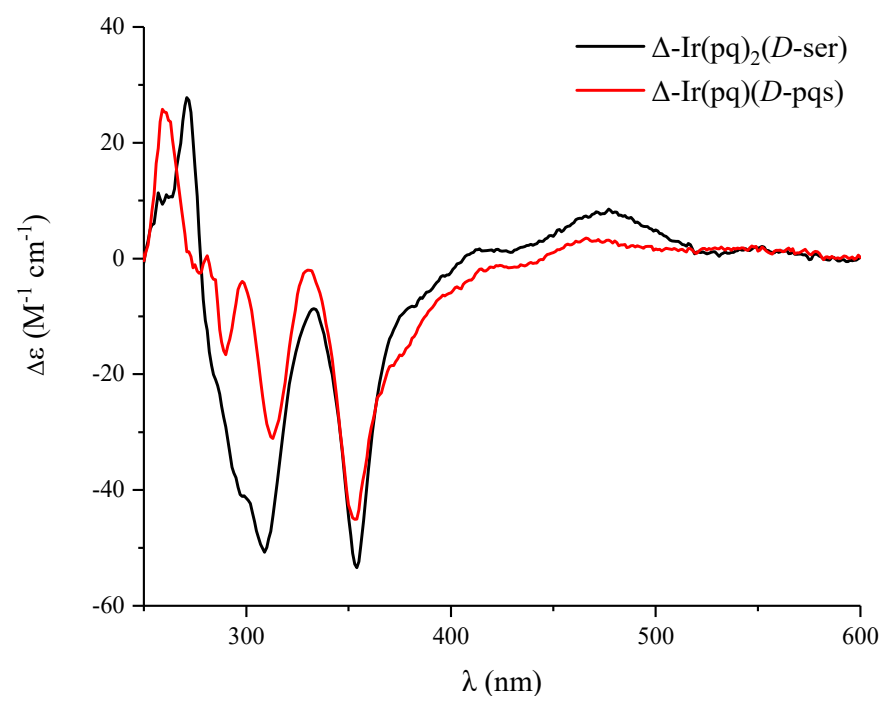

Figure S28. CD spectra of $\Delta$-[Ir(pq $)_{2}(D$-ser $\left.)\right]$ and $\Delta-[\operatorname{Ir}(\mathrm{pq})(D$-pqs $)]$ in $\mathrm{MeOH}(50 \mu \mathrm{M})$. 

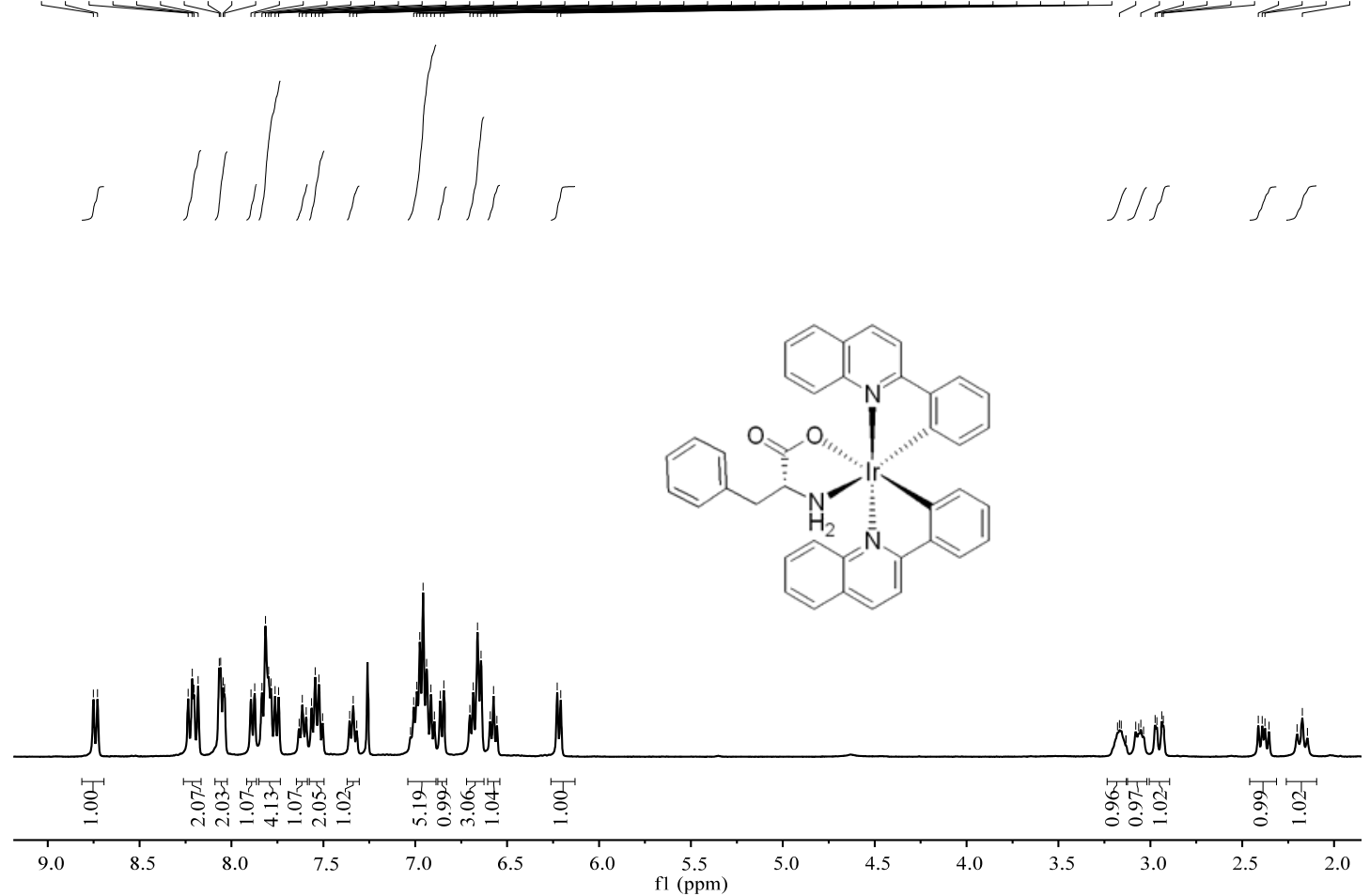

Figure S29. The ${ }^{1} \mathrm{H}$ NMR spectrum of $\Delta$-[Ir(pq) $\left.2(D-p a l)\right]$ in $\mathrm{CDCl}_{3}$.

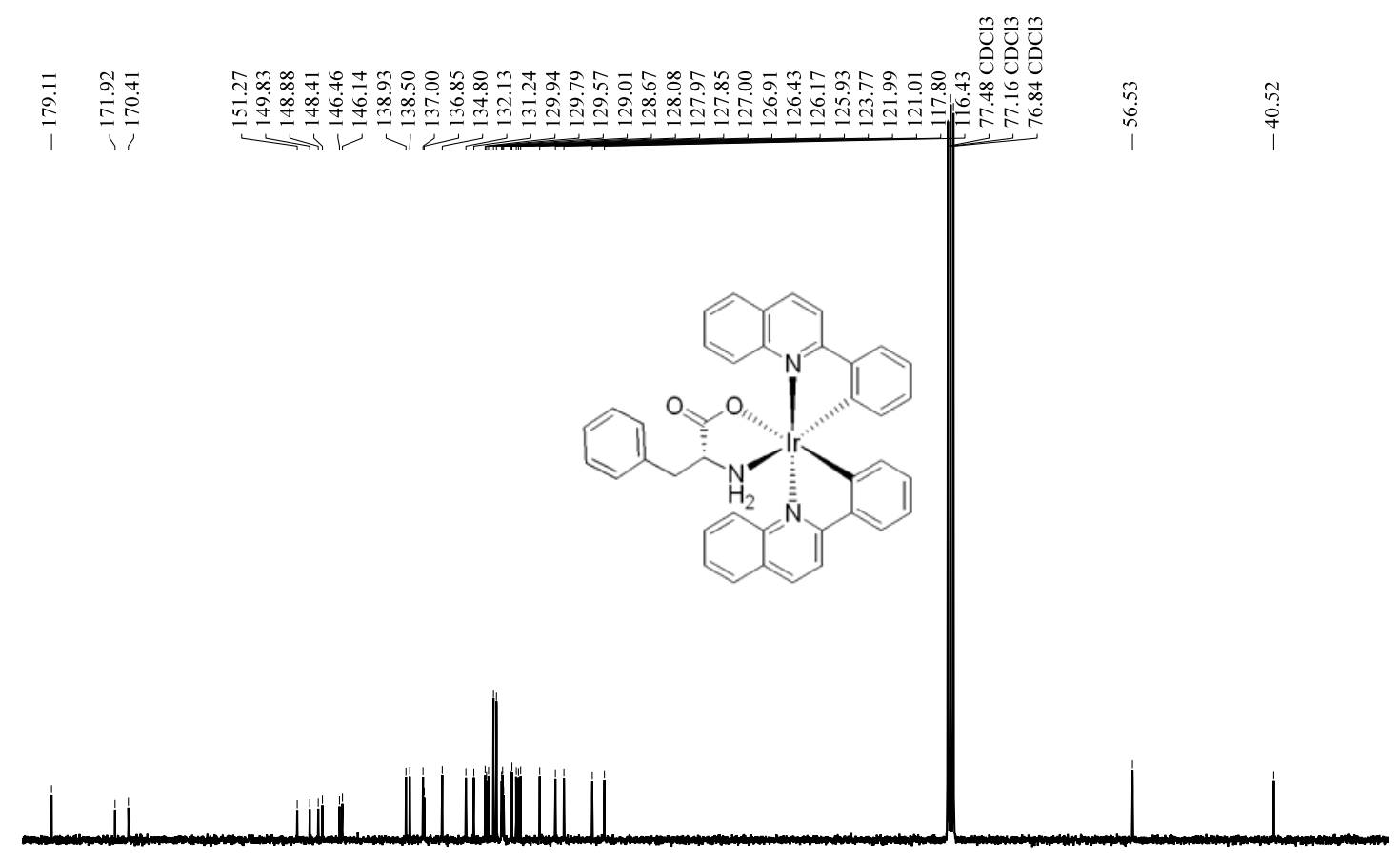

$\begin{array}{llllllllllllllllllllllllllllllllllllllllllllll}180 & 175 & 170 & 165 & 160 & 155 & 150 & 145 & 140 & 135 & 130 & 125 & 120 & 115 & 110 & 105 & 100 & 95 & 90 & 85 & 80 & 75 & 70 & 65 & 60 & 55 & 50 & 45 & 40 & 35 & 30\end{array}$

Figure S30. The ${ }^{13} \mathrm{C}$ NMR spectrum of $\Delta$-[Ir(pq) $\left.2(D-p a l)\right]$ in $\mathrm{CDCl}_{3}$. 


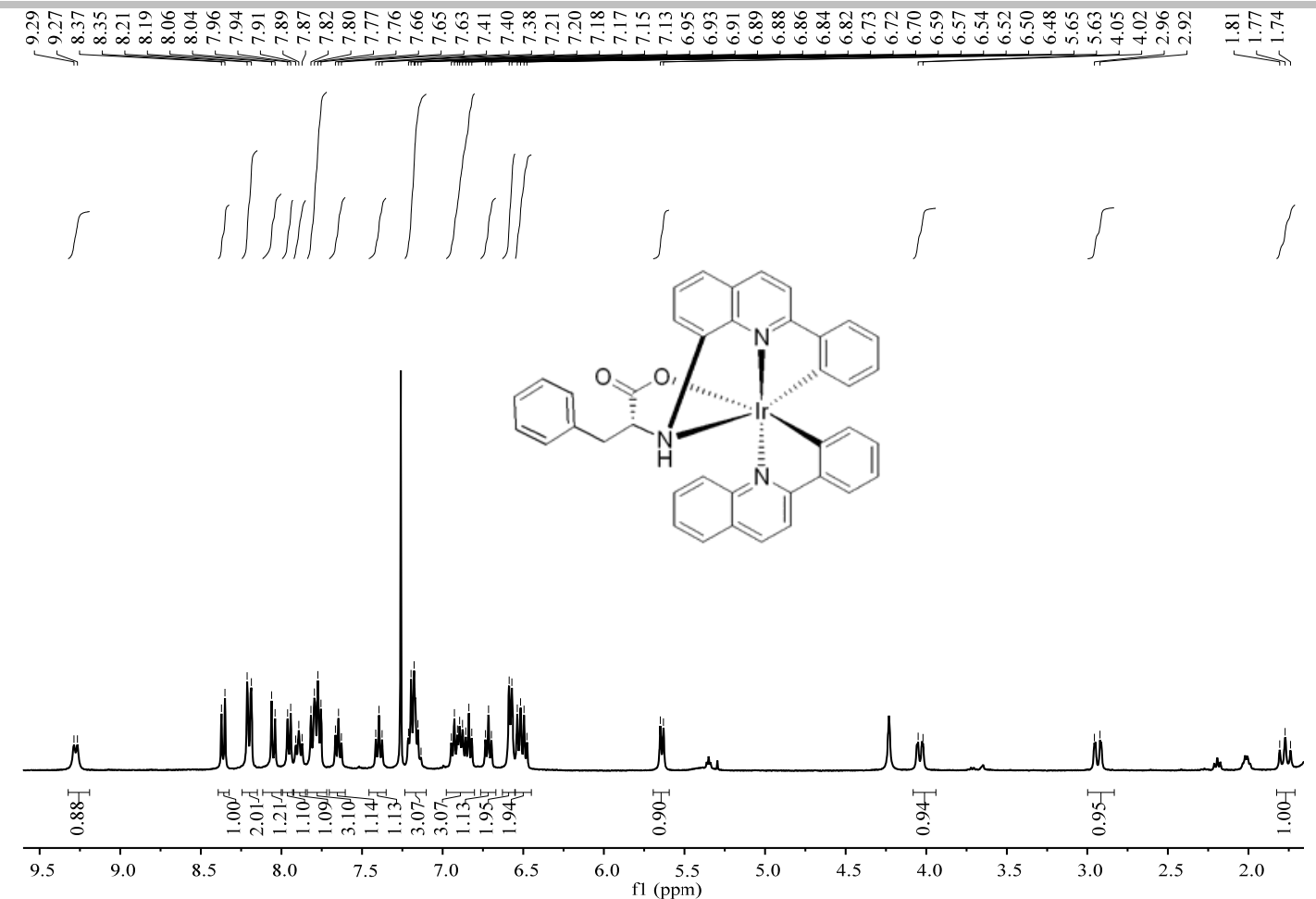

Figure S31. The ${ }^{1} \mathrm{H}$ NMR spectrum of $\Delta-[\operatorname{Ir}(\mathrm{pq})(D-\mathrm{pqpa})]$ in $\mathrm{CDCl}_{3}$.

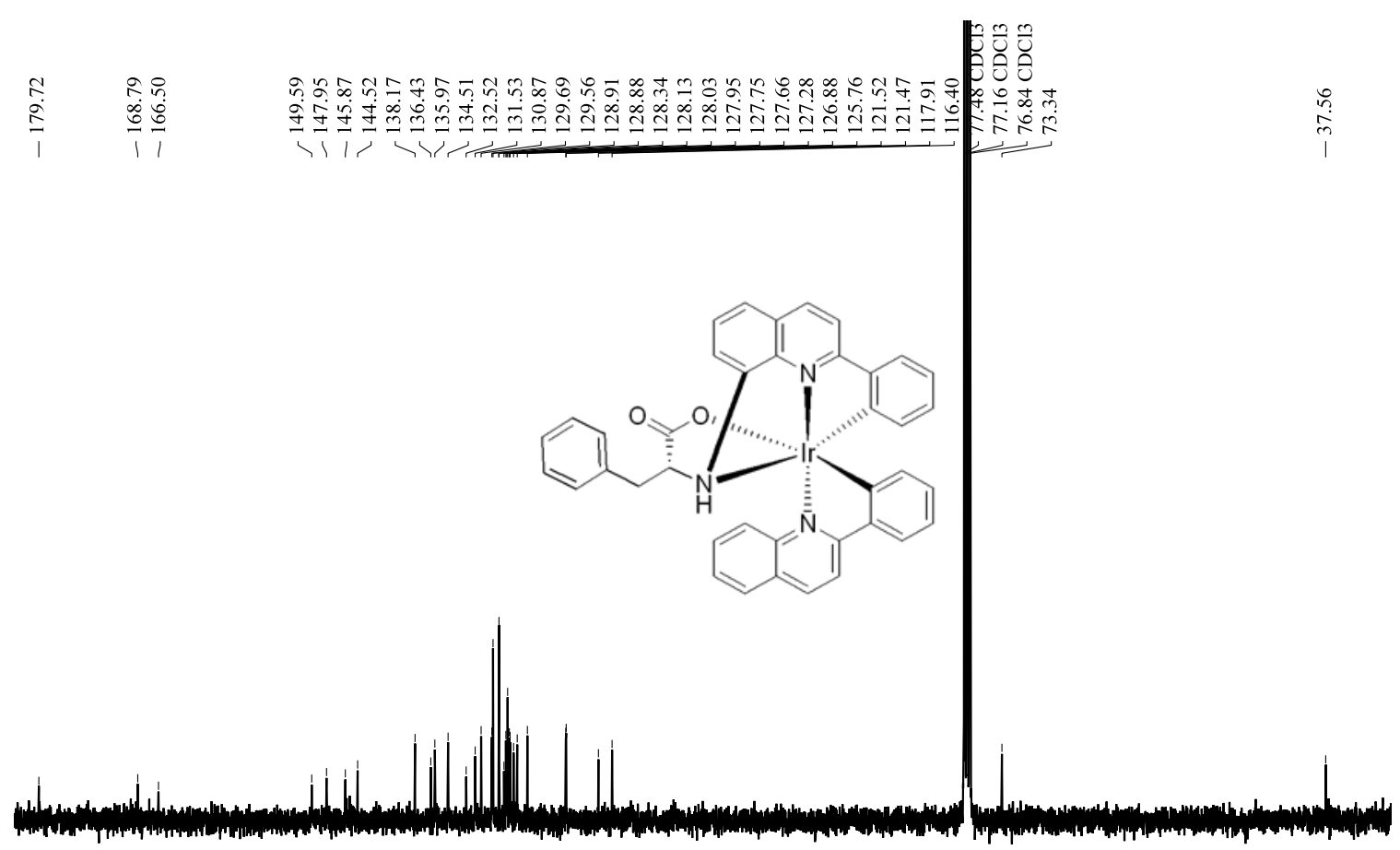

$\begin{array}{llllllllllllllllllllllllllllllllllll}180 & 175 & 170 & 165 & 160 & 155 & 150 & 145 & 140 & 135 & 130 & 125 & 120 & 115 & \begin{array}{c}110 \\ \mathrm{fl}(\mathrm{ppm})\end{array} & 100 & 95 & 90 & 85 & 80 & 75 & 70 & 65 & 60 & 55 & 50 & 45 & 40 & 35\end{array}$ Figure S32. The ${ }^{13} \mathrm{C}$ NMR spectrum of $\Delta-[\operatorname{Ir}(\mathrm{pq})(D$-pqpa $)]$ in $\mathrm{CDCl}_{3}$. 


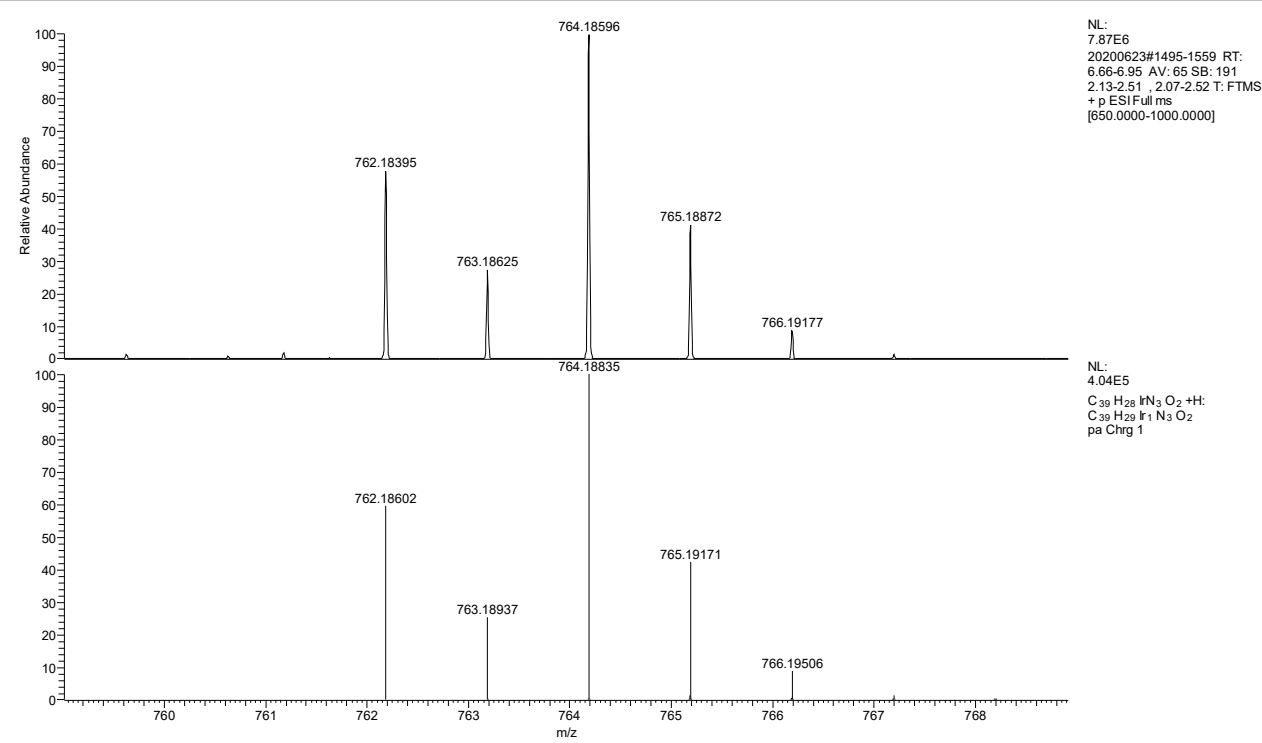

Figure S33. Comparison of HRMS of $\Delta$-[Ir(pq)(D-pqpa)] at m/z $764.18596\left([\mathrm{M}+\mathrm{H}]^{+}\right)(\mathrm{up})$ and calculated for $\Delta-[\operatorname{Ir}(\mathrm{pq})(D-\mathrm{pqpa})]$ $\left(\left[\mathrm{C}_{39} \mathrm{H}_{28} \mathrm{IrN}_{3} \mathrm{O}_{2}+\mathrm{H}\right]^{+}, 764.18835\right.$, down).

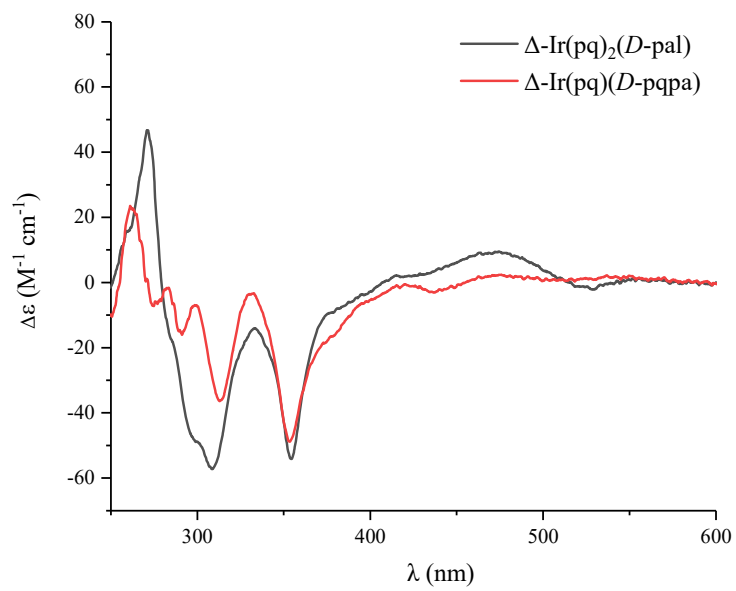

Figure S34. CD spectra of $\Delta$-[Ir(pq $)_{2}(D$-pal) $]$ and $\Delta$-[Ir(pq)(D-pqpa) $]$ in $\mathrm{MeOH}(50 \mu \mathrm{M})$. 

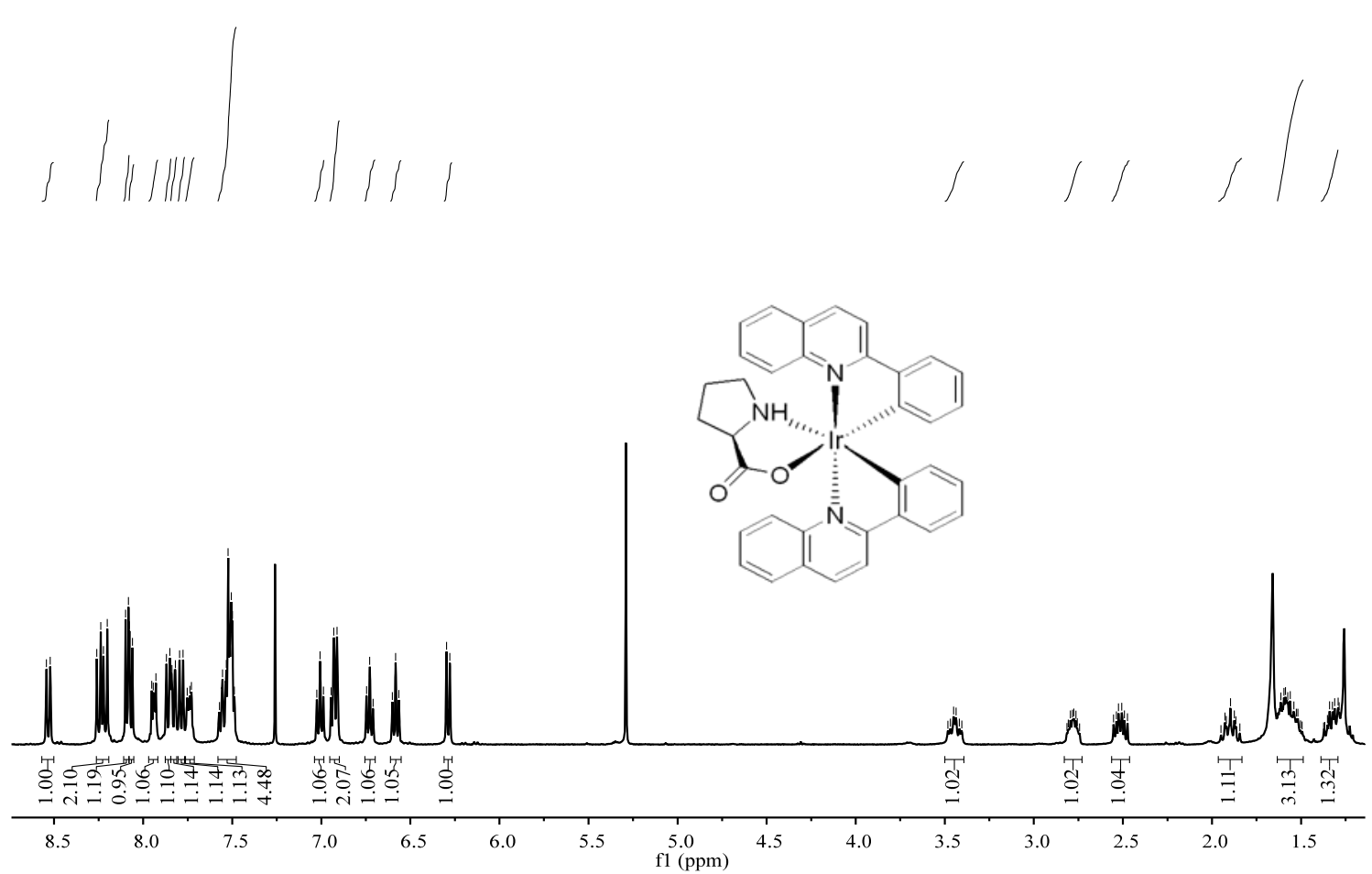

Figure S35. The ${ }^{1} \mathrm{H}$ NMR spectrum of $\Delta-\left[\operatorname{Ir}(\mathrm{pq})_{2}(D\right.$-pro $\left.)\right]$ in $\mathrm{CDCl}_{3}$.
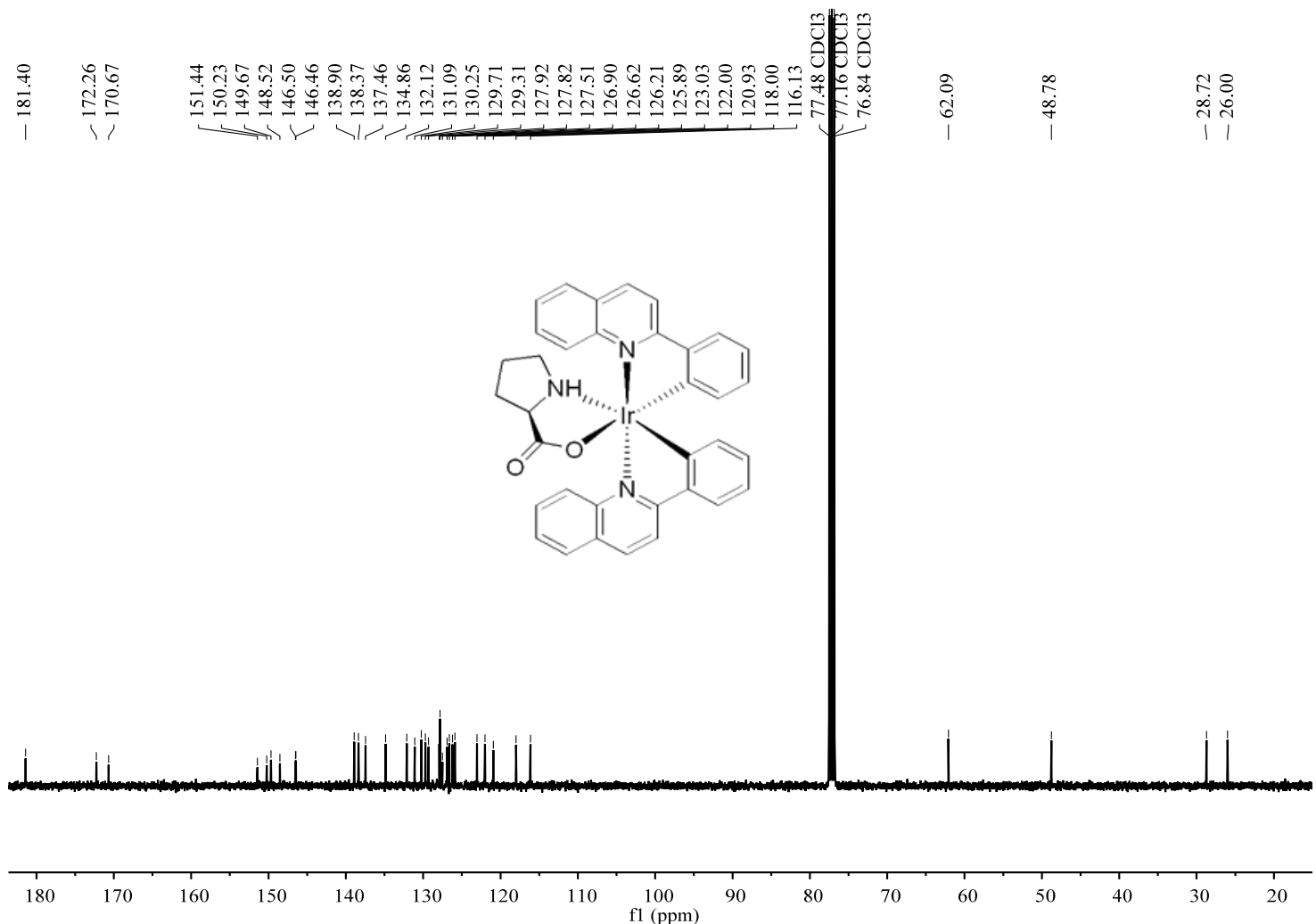

Figure S36. The ${ }^{13} \mathrm{C}$ NMR spectrum of $\Delta$-[Ir(pq) $2(D$-pro $\left.)\right]$ in $\mathrm{CDCl}_{3}$. 


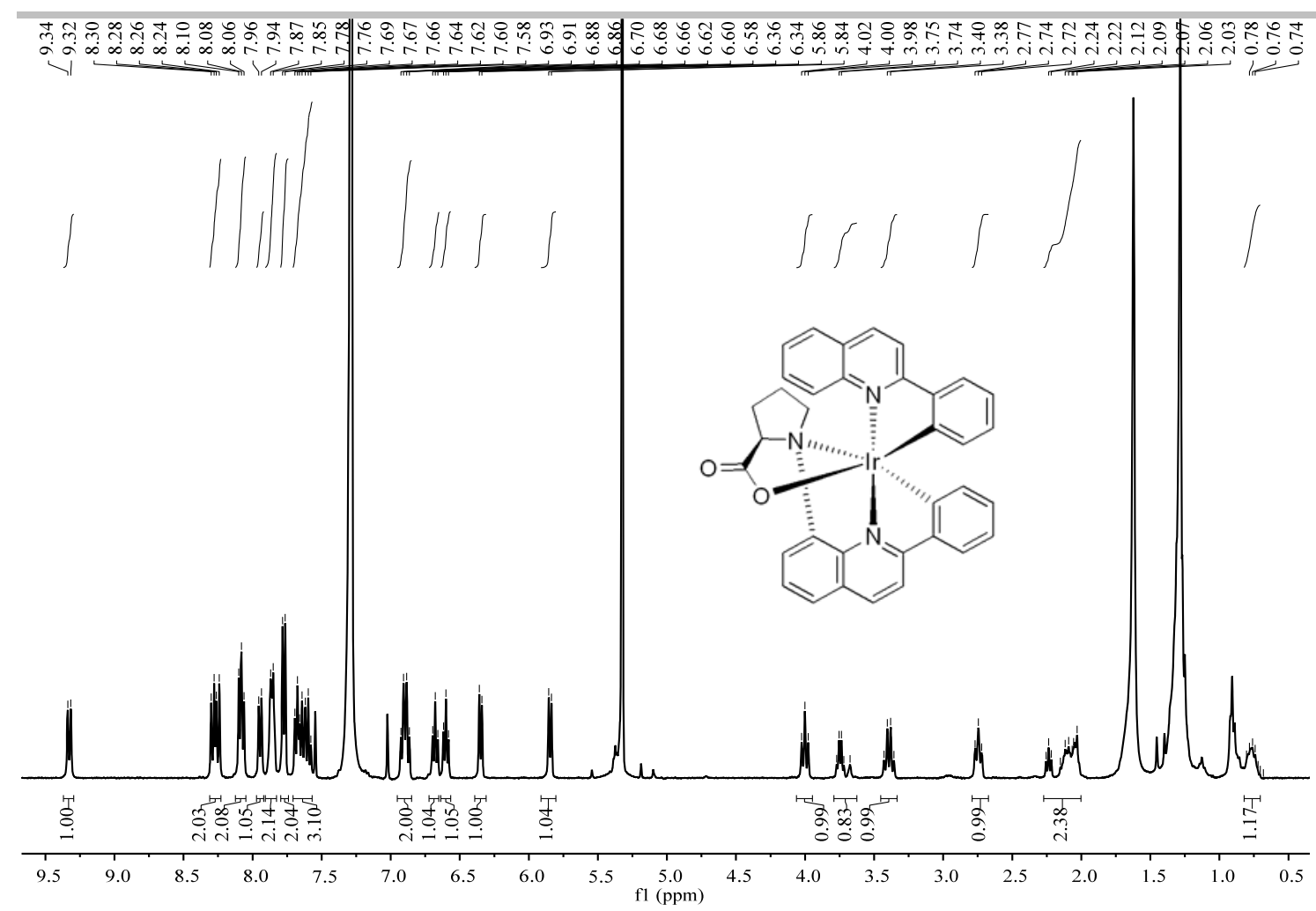

Figure S37. The ${ }^{1} \mathrm{H}$ NMR spectrum of $\Delta-[\operatorname{Ir}(\mathrm{pq})(D-p q p)]$ in $\mathrm{CDCl}_{3}$.

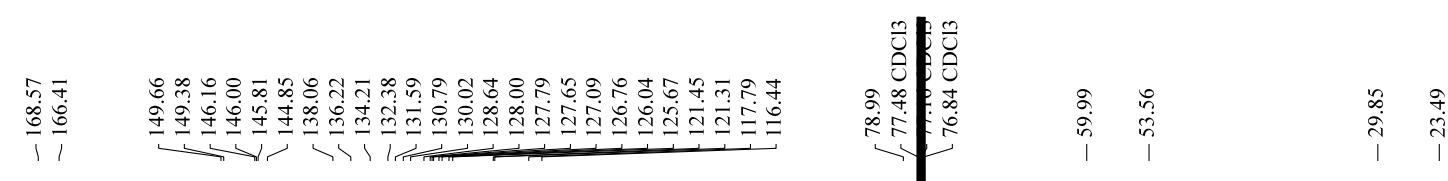

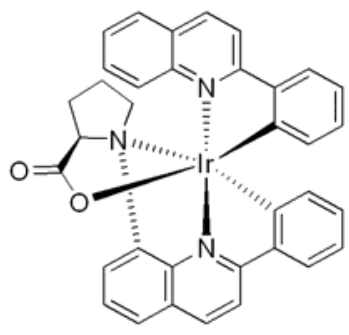

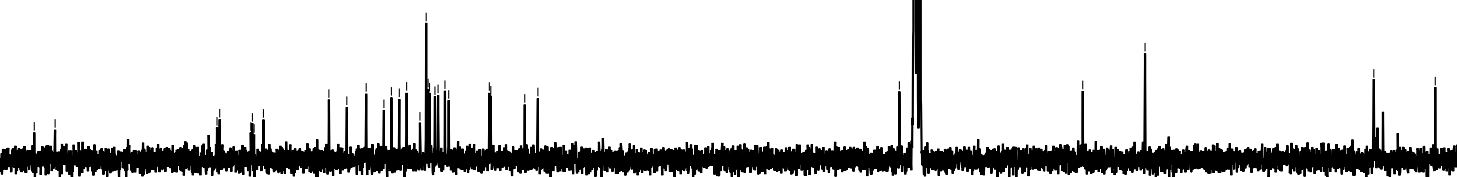

$\begin{array}{llllllllllllllllllllllllllllllllllll}170 & 165 & 160 & 155 & 150 & 145 & 140 & 135 & 130 & 125 & 120 & 115 & 110 & 105 & 100 & 95 & 90 & 85 & 80 & 75 & 70 & 65 & 60 & 55 & 50 & 45 & 40 & 35 & 30 & 25\end{array}$

Figure S38. The ${ }^{13} \mathrm{C}$ NMR spectrum of $\Delta-[\operatorname{Ir}(\mathrm{pq})(D-\mathrm{pqp})]$ in $\mathrm{CDCl}_{3}$. 


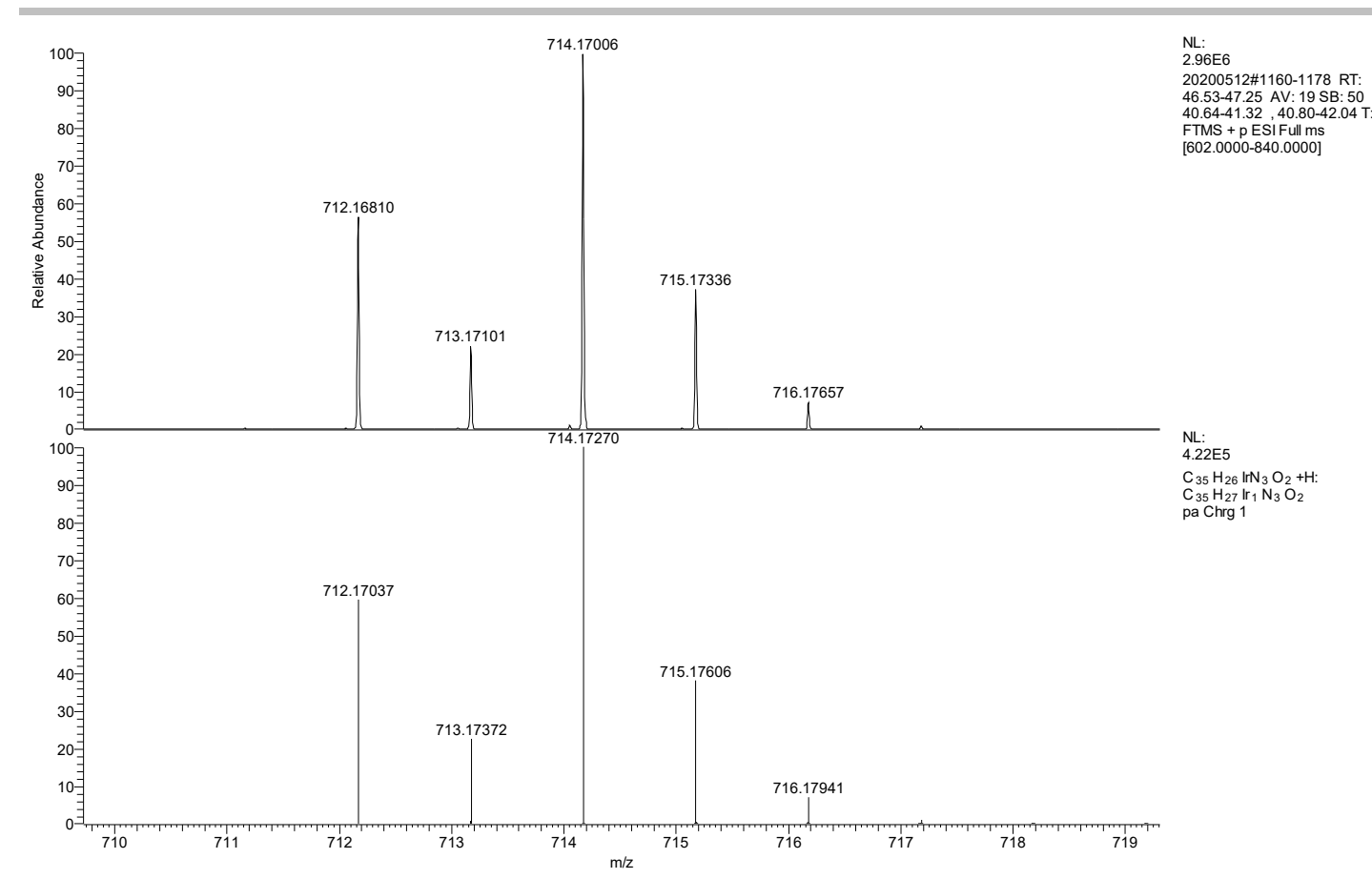

Figure S39. Comparison of HRMS of $\Delta$-[Ir(pq)(D-pqp)] at m/z $714.17006\left([\mathrm{M}+\mathrm{H}]^{+}\right)(\mathrm{up})$ and calculated for $\Delta$-[Ir(pq)(D-pqp)] $\left(\left[\mathrm{C}_{35} \mathrm{H}_{26} \mathrm{IrN}_{3} \mathrm{O}_{2}+\mathrm{H}\right]^{+}, 714.17270\right.$, down).

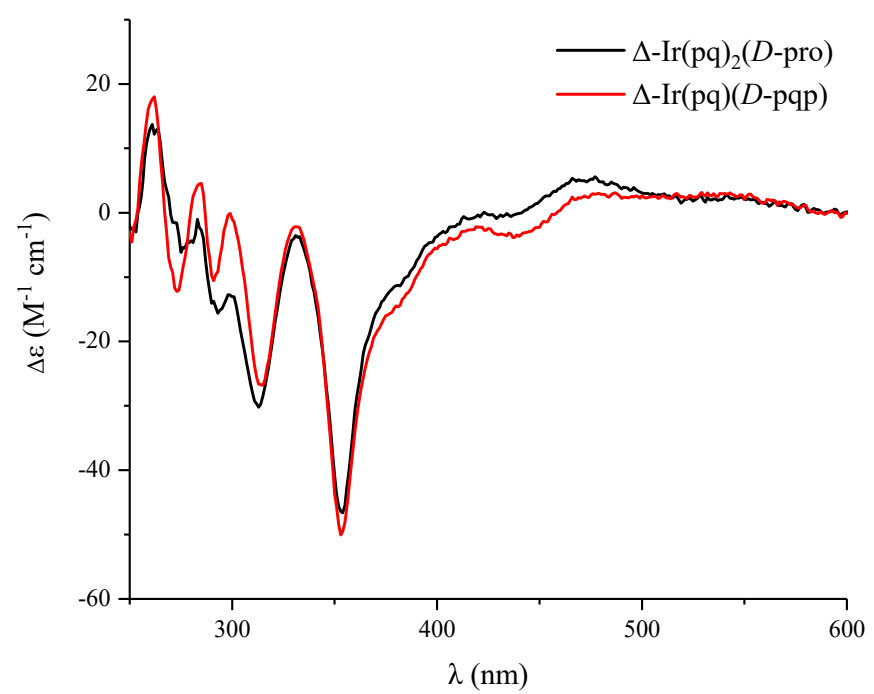

Figure S40. CD spectra of $\Delta-\left[\operatorname{Ir}(\mathrm{pq})_{2}(D\right.$-pro $\left.)\right]$ and $\Delta$-[Ir(pq)(D-pqp) $]$ in $\mathrm{MeOH}(50 \mu \mathrm{M})$. 


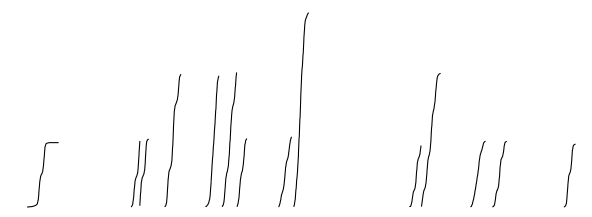<smiles>CC(C)(NCC(=O)O)c1ccccc1-c1cccc2ccccc12</smiles><smiles>C[Te](O)(NCC(=O)Nc1ccccc1)(c1ccccc1)c1ccccc1-c1cccc2ccccc12</smiles>

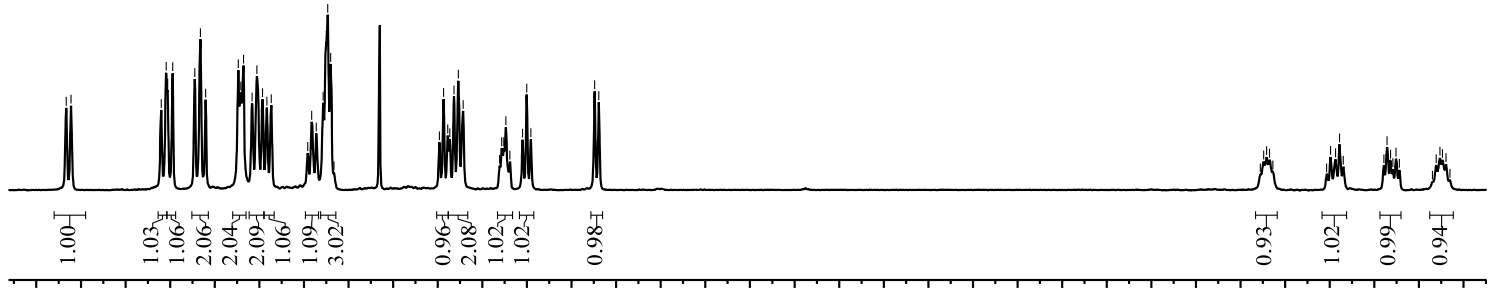

$\begin{array}{llllllllllllllllllllllllllllllllllllllllll}8.8 & 8.6 & 8.4 & 8.2 & 8.0 & 7.8 & 7.6 & 7.4 & 7.2 & 7.0 & 6.8 & 6.6 & 6.4 & 6.2 & 6.0 & 5.8 & 5.6 & 5.4 & 5.2 & 5.0 & 4.8 & 4.6 & 4.4 & 4.2 & 4.0 & 3.8 & 3.6 & 3.4 & 3.2 & 3.0 & 2.8 & 2.6 & 2.4\end{array}$

Figure $\mathbf{S 4 1}$. The ${ }^{1} \mathrm{H}$ NMR spectrum of $\Delta-\left[\operatorname{Ir}(\mathrm{pq})_{2}(\mathrm{gly})\right]$ in $\mathrm{CDCl}_{3}$.<smiles></smiles><smiles></smiles>

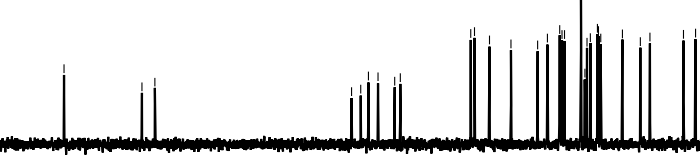

$\begin{array}{llllllllllllllllllllllllllllllllllll}185 & 180 & 175 & 170 & 165 & 160 & 155 & 150 & 145 & 140 & 135 & 130 & 125 & 120 & 115 & 110 & 105 & 100 & 95 & 90 & 85 & 80 & 75 & 70 & 65 & 60 & 55 & 50 & 45 & 40\end{array}$ f1 (ppm)

Figure S42. The ${ }^{13} \mathrm{C}$ NMR spectrum of $\Delta-\left[\operatorname{Ir}(\mathrm{pq})_{2}(\mathrm{gly})\right]$ in $\mathrm{CDCl}_{3}$. 

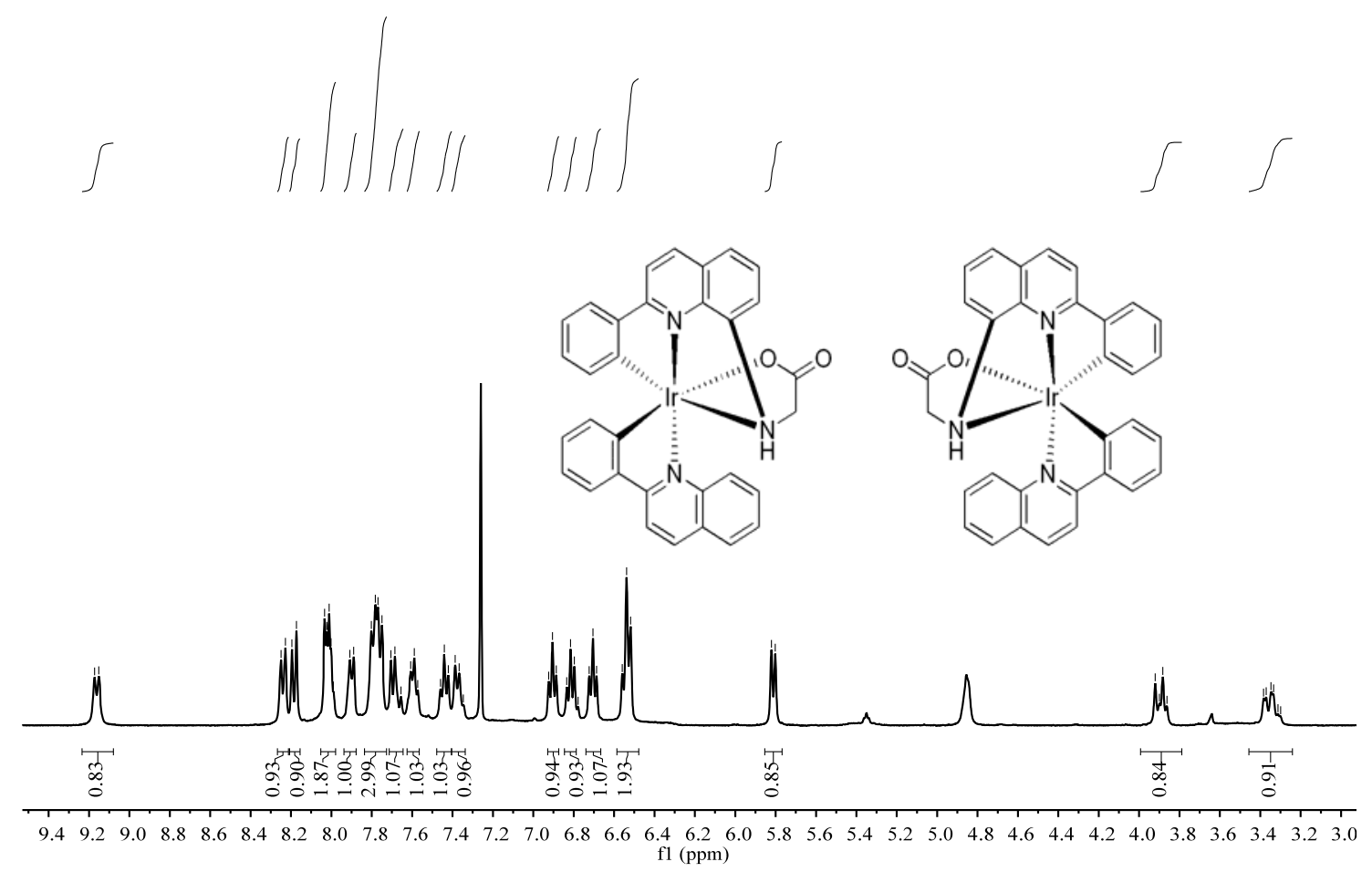

Figure S43. The ${ }^{1} \mathrm{H}$ NMR spectrum of $\Delta-[\operatorname{Ir}(\mathrm{pq})(\mathrm{pqg})]$ in $\mathrm{CDCl}_{3}$.

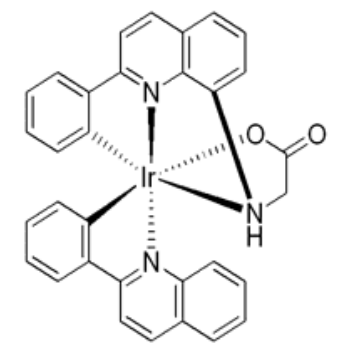

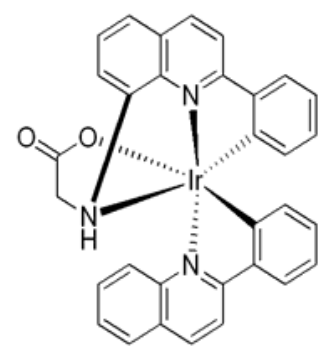

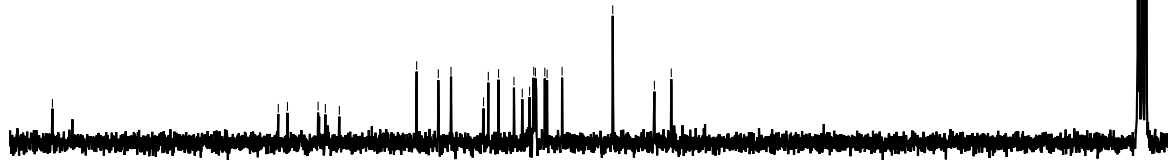

$\begin{array}{llllllllllllllllllllllllllllllllllllll}170 & 165 & 160 & 155 & 150 & 145 & 140 & 135 & 130 & 125 & 120 & 115 & 110 & 105 & 100 & 95 & 90 & 85 & 80 & 75 & 70 & 65 & 60 & 55 & 50\end{array}$

Figure S44. The ${ }^{13} \mathrm{C}$ NMR spectrum of $\Delta-[\operatorname{Ir}(\mathrm{pq})(\mathrm{pqg})]$ in $\mathrm{CDCl}_{3}$. 


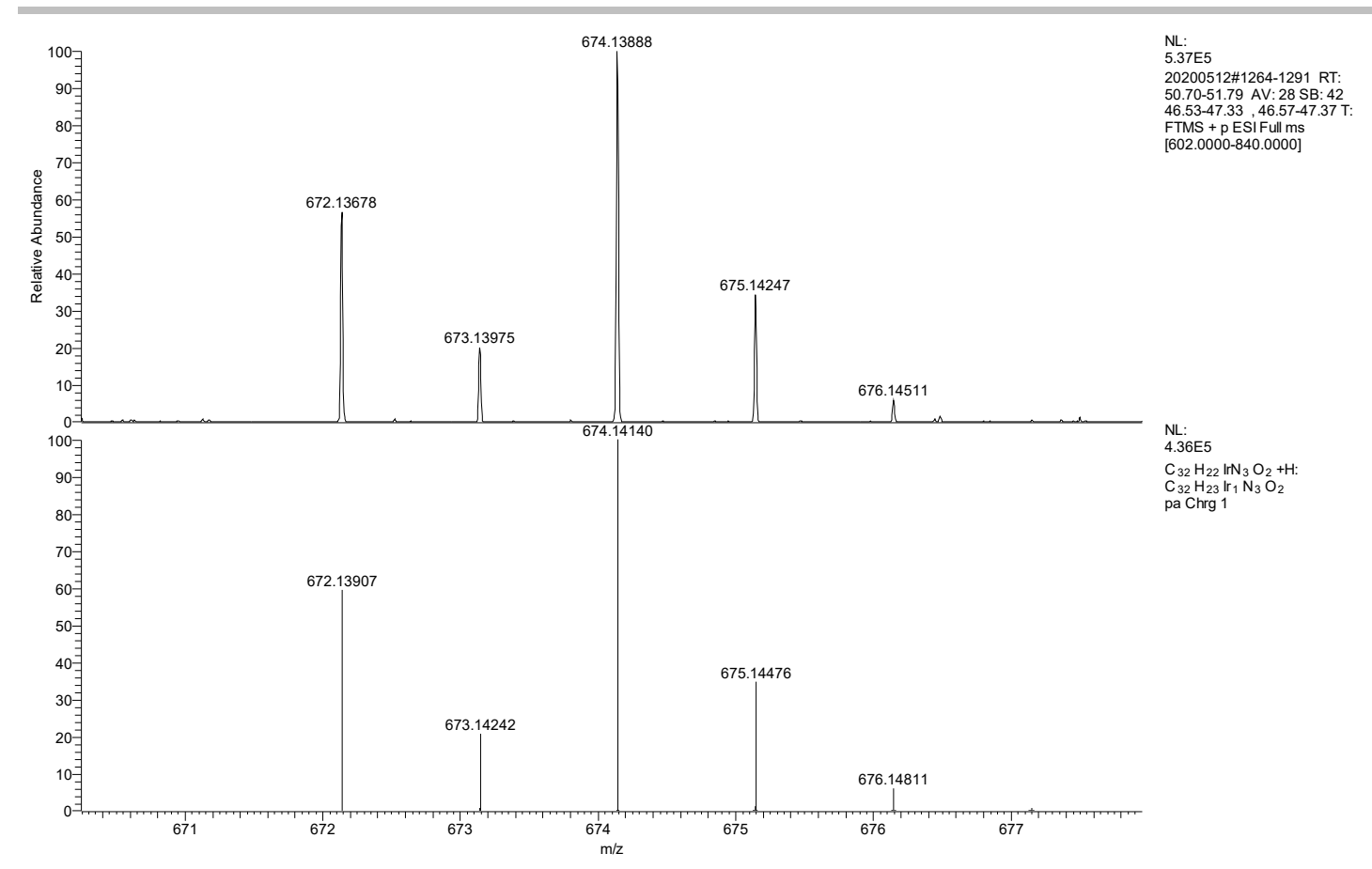

Figure S45. Comparison of HRMS of $\Delta$-[Ir(pq)(pqg)] at m/z $674.13888\left([\mathrm{M}+\mathrm{H}]^{+}\right)(\mathrm{up})$ and calculated for $\Delta$-[Ir(pq)(pqg)] $\left(\left[\mathrm{C}_{32} \mathrm{H}_{22} \mathrm{IrN}_{3} \mathrm{O}_{2}+\mathrm{H}\right]^{+}, 674.14140\right.$, down).
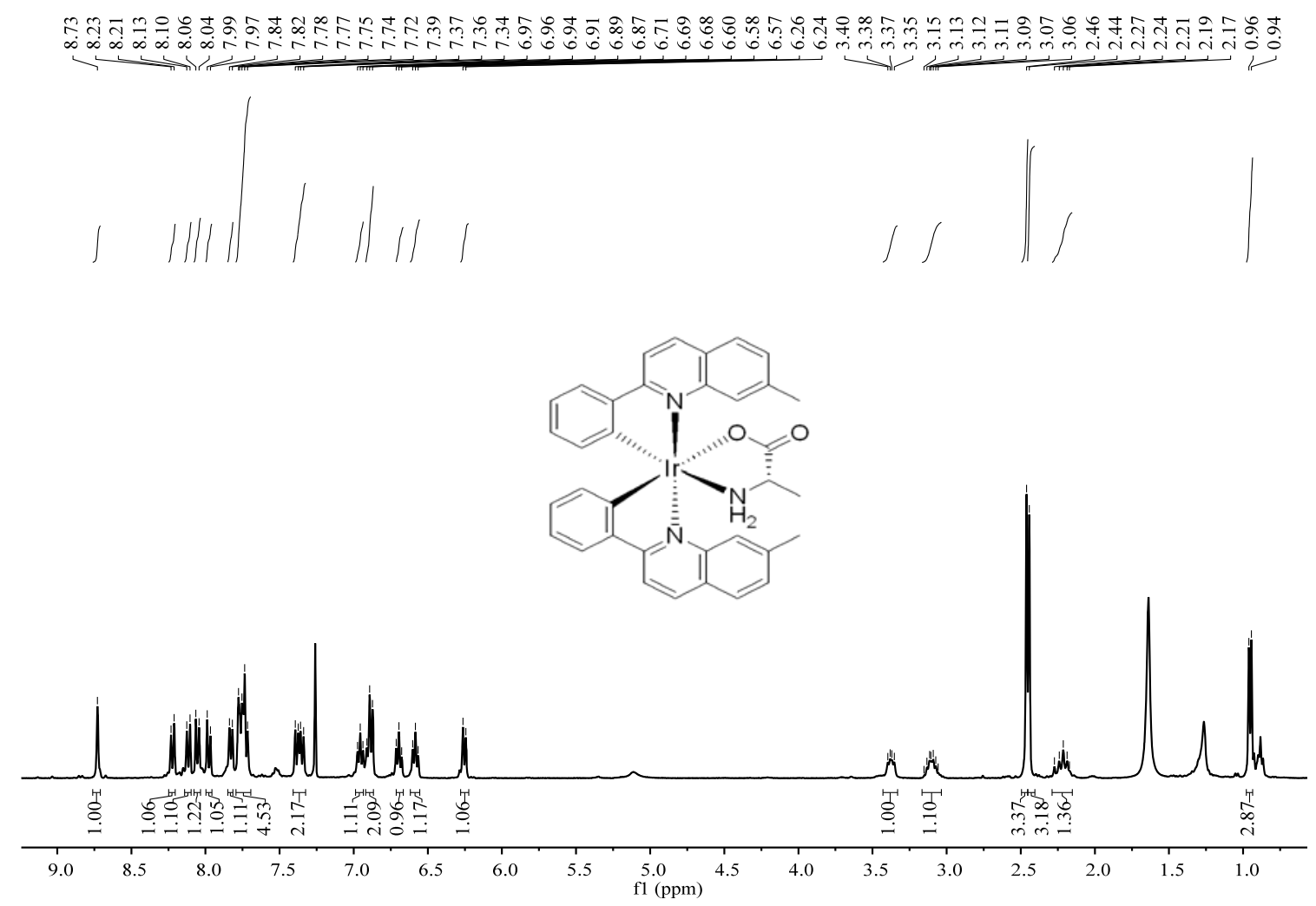

Figure S46. The ${ }^{1} \mathrm{H}$ NMR spectrum of $\Lambda$ - $\left[\operatorname{Ir}(\mathrm{Mpq})_{2}(L\right.$-ala $\left.)\right]$ in $\mathrm{CDCl}_{3}$. 

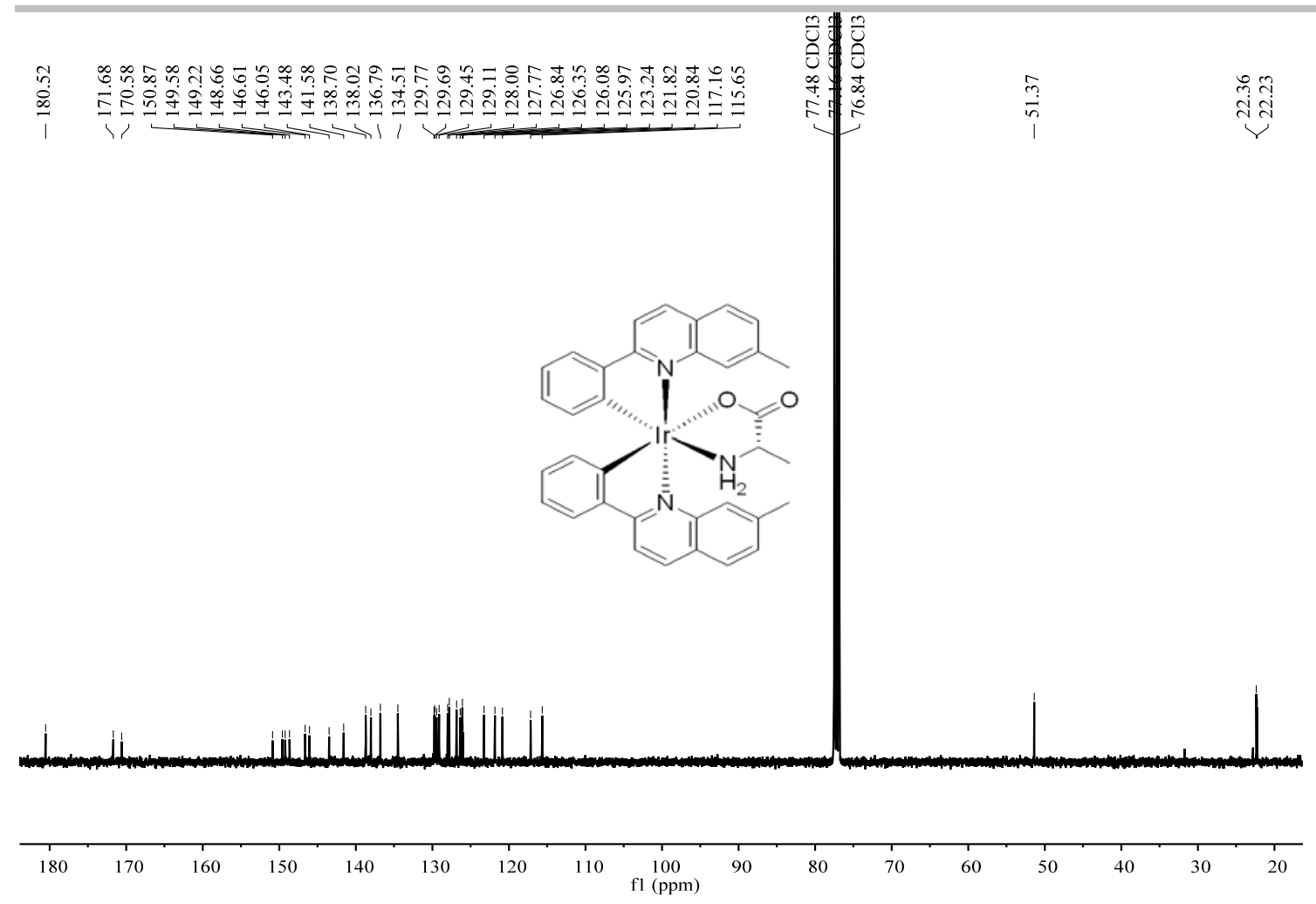

Figure S47. The ${ }^{13} \mathrm{C}$ NMR spectrum of $\Lambda$-[ $\left[\mathrm{r}(\mathrm{Mpq})_{2}(L\right.$-ala $\left.)\right]$ in $\mathrm{CDCl}_{3}$.

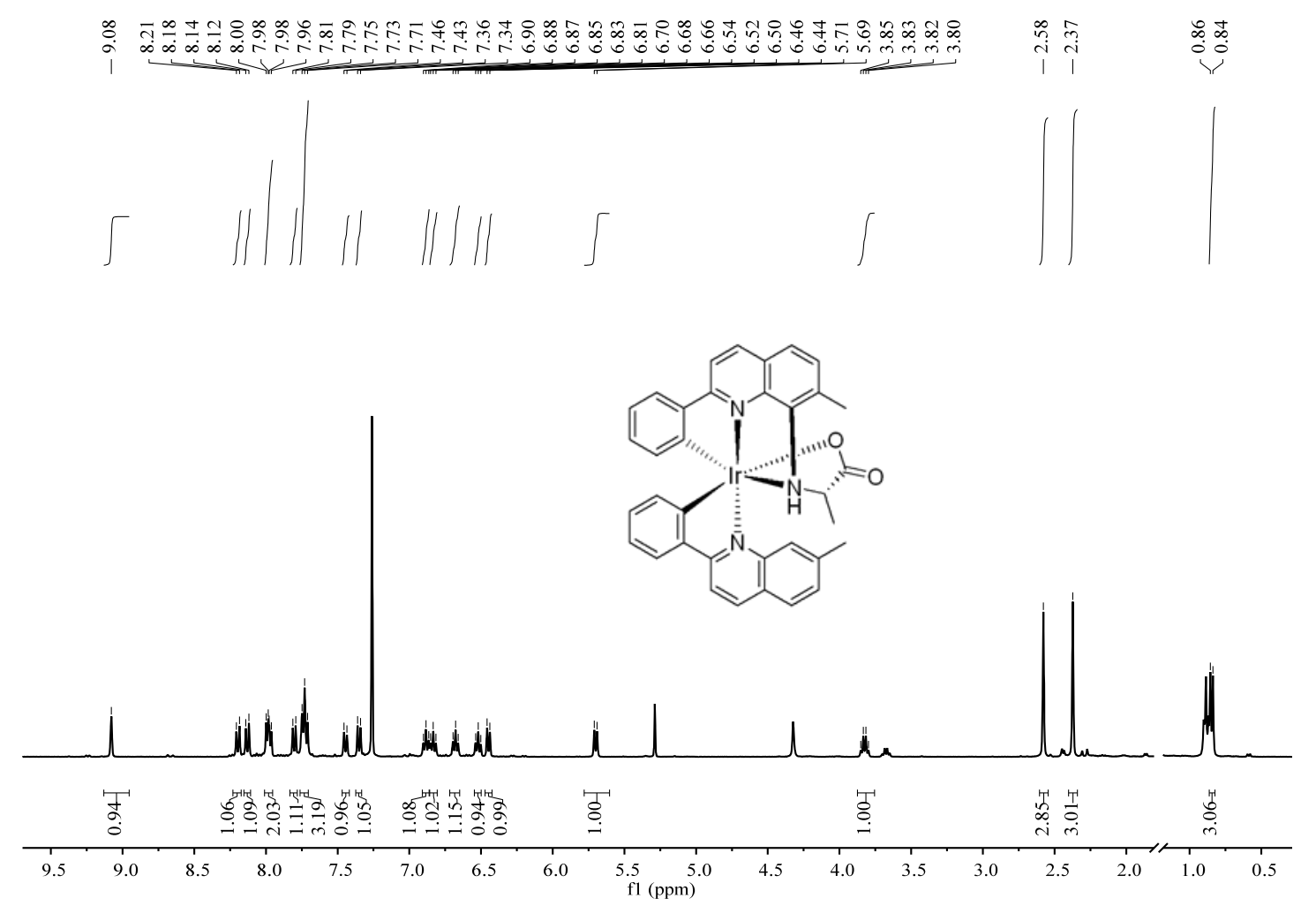

Figure S48. The ${ }^{1} \mathrm{H}$ NMR spectrum of $\Lambda$-[ $\left.\operatorname{Ir}(\mathrm{Mpq})(L-\mathrm{Mpqa})\right]$ in $\mathrm{CDCl}_{3}$. 


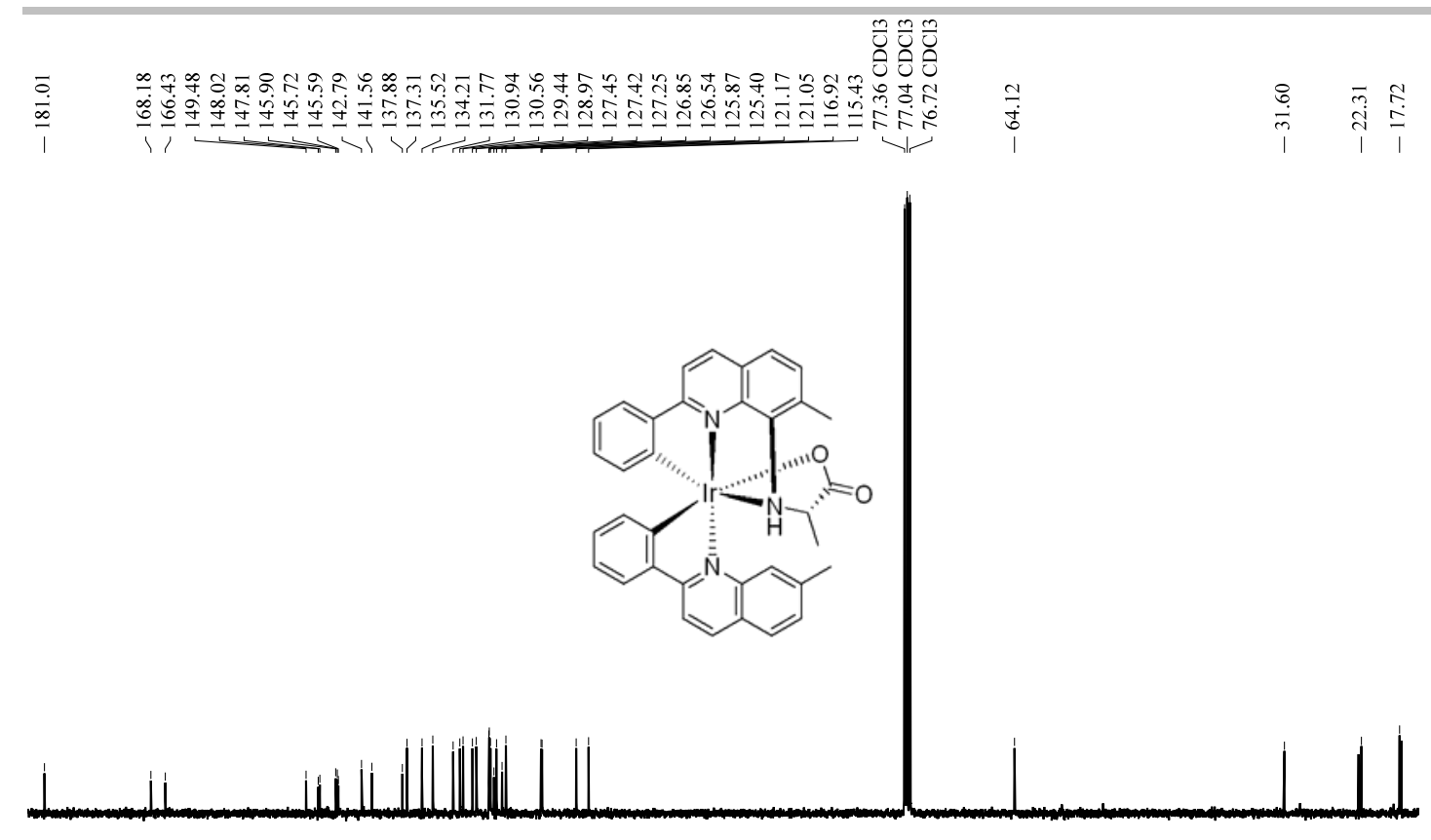

\begin{tabular}{|c|c|c|c|c|c|c|c|c|c|c|c|c|c|c|c|c|}
\hline 180 & 170 & 160 & 150 & 140 & 130 & 120 & 110 & $\begin{array}{c}100 \\
\mathrm{fl}(\mathrm{ppm})\end{array}$ & 90 & 80 & 70 & 60 & 50 & 40 & 30 & 20 \\
\hline
\end{tabular}

Figure S49. The ${ }^{13} \mathrm{C}$ NMR spectrum of $\Lambda$-[Ir(Mpq) $\left.(L-M p q a)\right]$ in $\mathrm{CDCl}_{3}$.

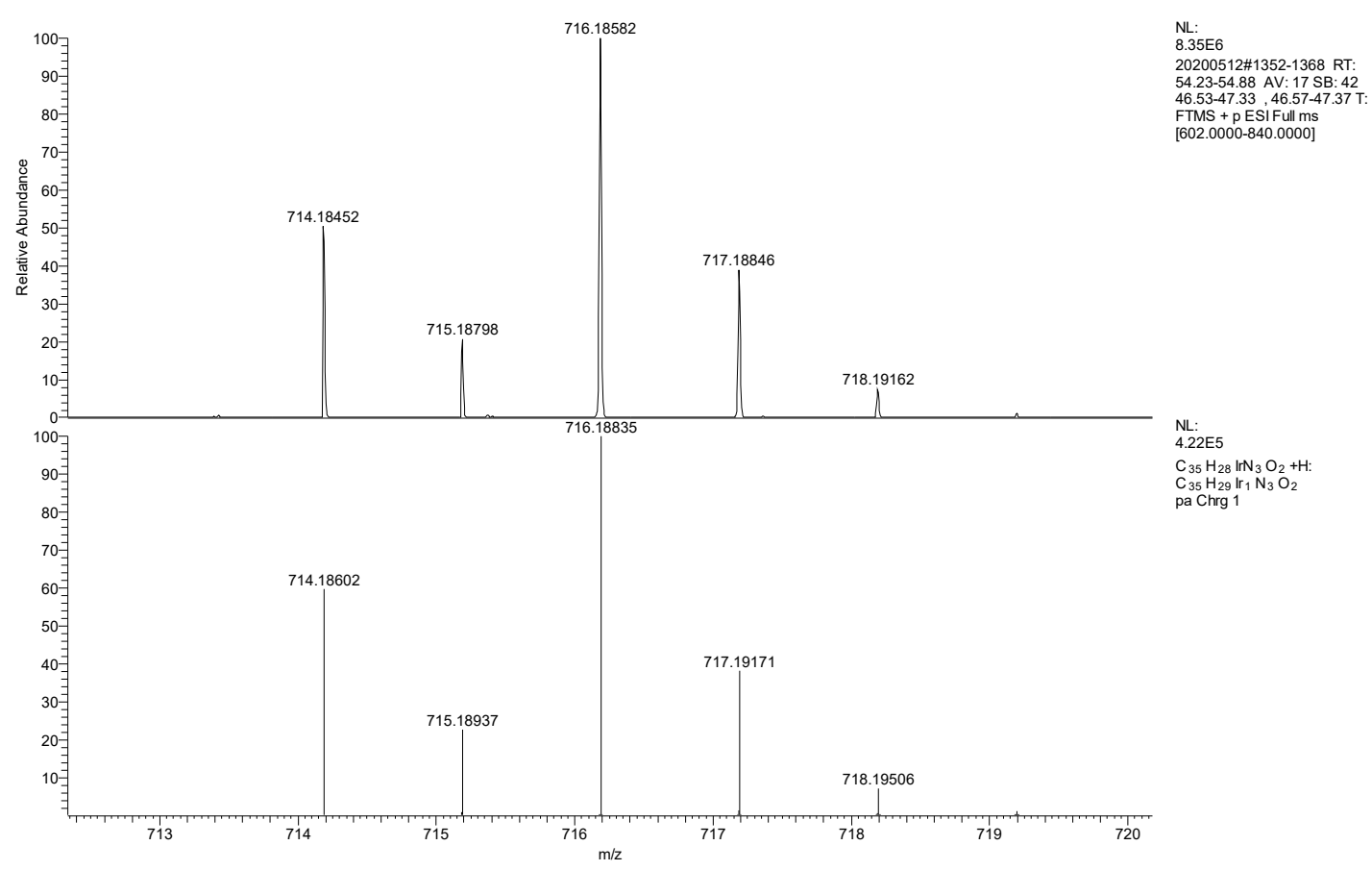

Figure S50. Comparison of HRMS of $\Lambda$-[ $\operatorname{Ir}(\mathrm{Mpq})(L-\mathrm{Mpqa})]$ at $\mathrm{m} / \mathrm{z} 716.18582\left([\mathrm{M}+\mathrm{H}]^{+}\right)$(up) and calculated for $\Lambda$-[ $\operatorname{Ir}(\mathrm{Mpq})(L-$ Mpqa) $]\left(\left[\mathrm{C}_{35} \mathrm{H}_{28} \mathrm{IrN}_{3} \mathrm{O}_{2}+\mathrm{H}\right]^{+}, 716.18835\right.$, down). 


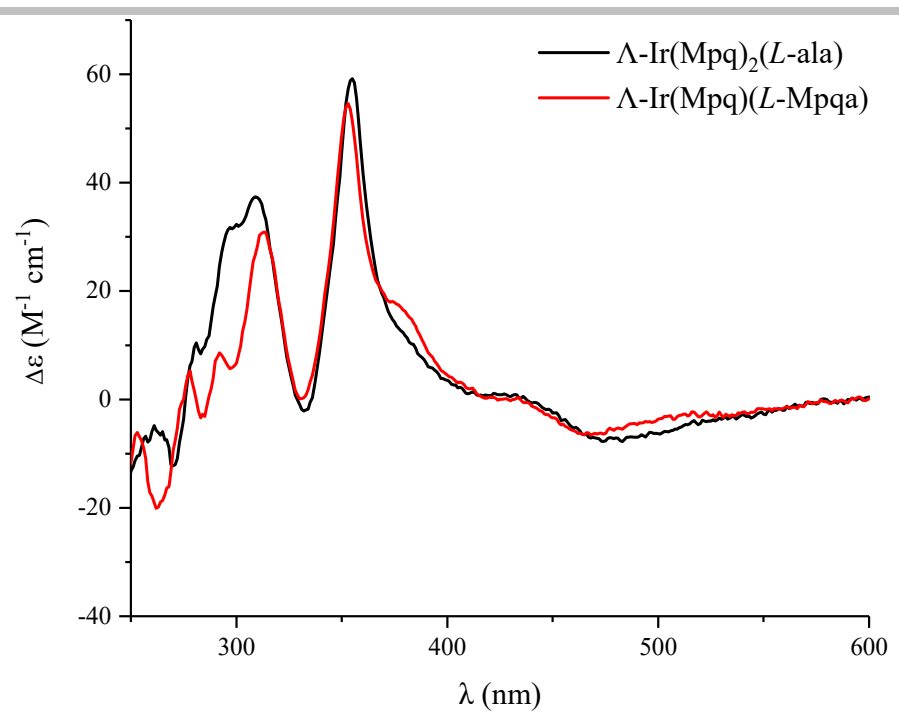

Figure S51. CD spectra of $\Lambda$-[Ir(Mpq $)_{2}(L$-ala $\left.)\right]$ and $\Lambda$-[Ir(Mpq $\left.)(L-M p q a)\right]$ in $\mathrm{MeOH}(50 \mu \mathrm{M})$.

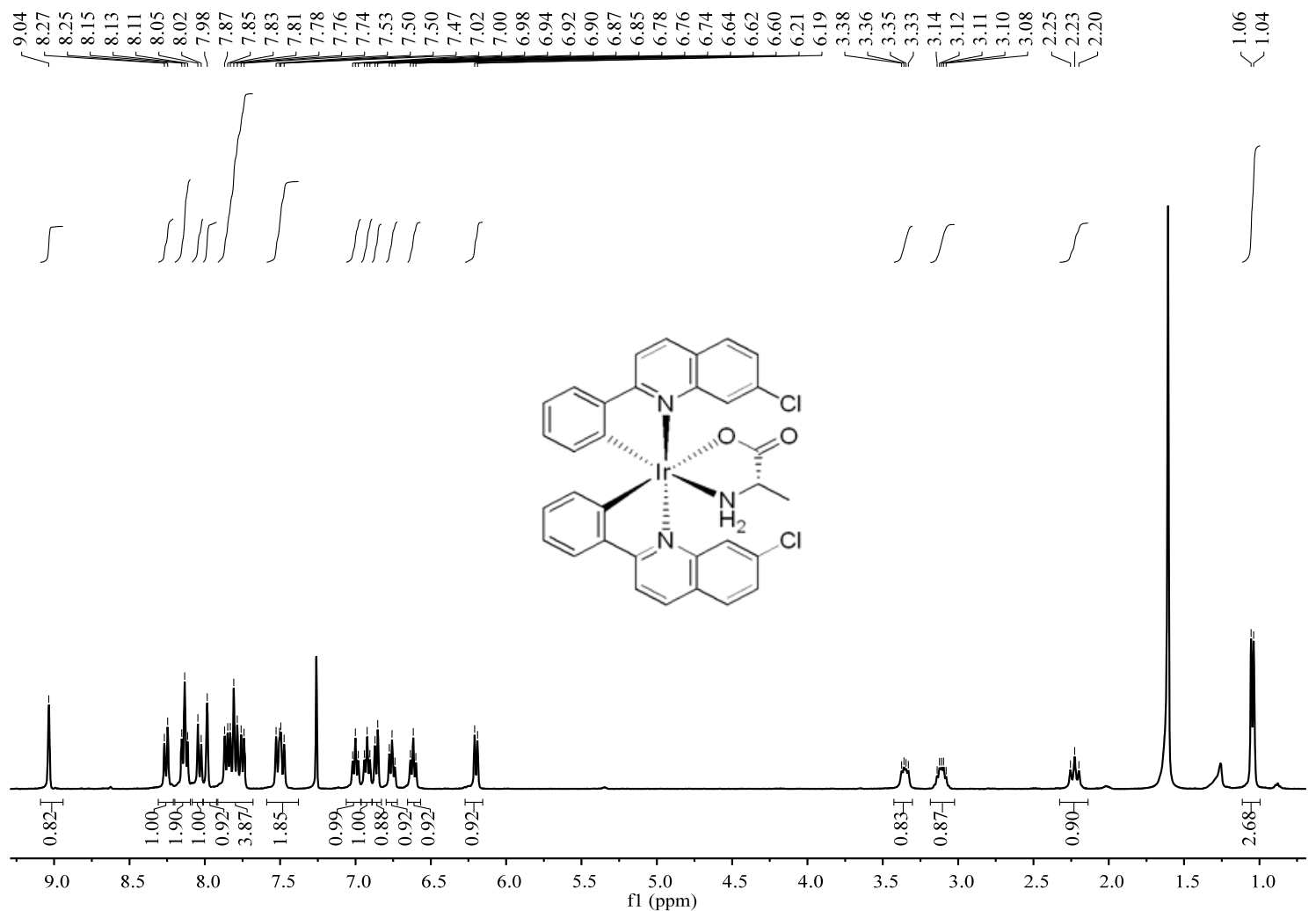

Figure S52. The ${ }^{1} \mathrm{H}$ NMR spectrum of $\Lambda$ - $\left[\operatorname{Ir}(\mathrm{Cpq})_{2}(L\right.$-ala $\left.)\right]$ in $\mathrm{CDCl}_{3}$. 


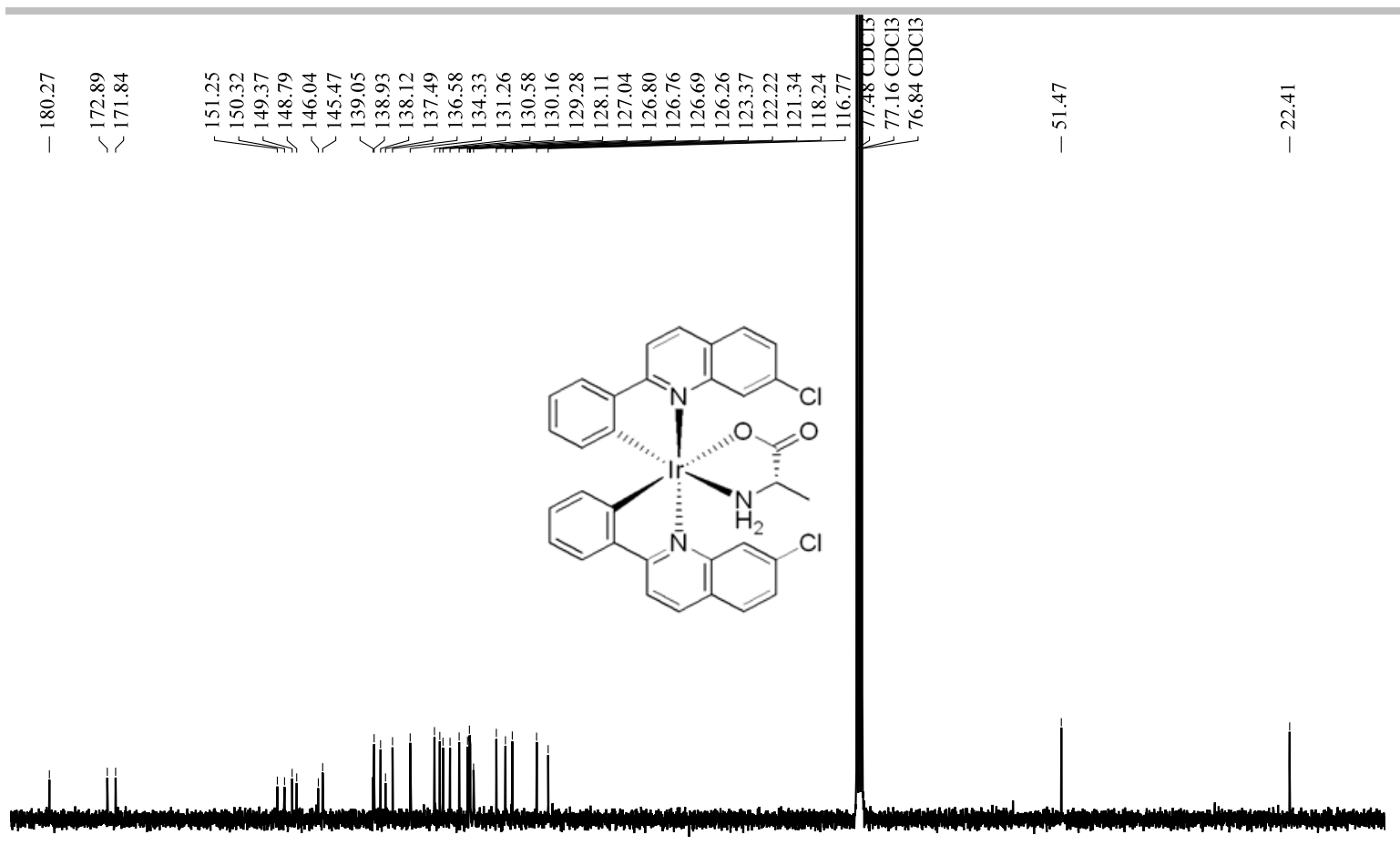

\begin{tabular}{lllllllllllllllll}
\hline 180 & 170 & 160 & 150 & 140 & 130 & 120 & 110 & $\underset{\mathrm{f} 1(\mathrm{ppm})}{100}$ & 90 & 80 & 70 & 60 & 50 & 40 & 30 & 20
\end{tabular}

Figure S53. The ${ }^{13} \mathrm{C}$ NMR spectrum of $\Lambda$-[Ir(Cpq $)_{2}(L$-ala $\left.)\right]$ in $\mathrm{CDCl}_{3}$.

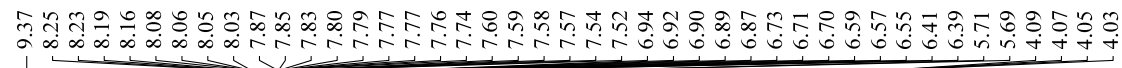
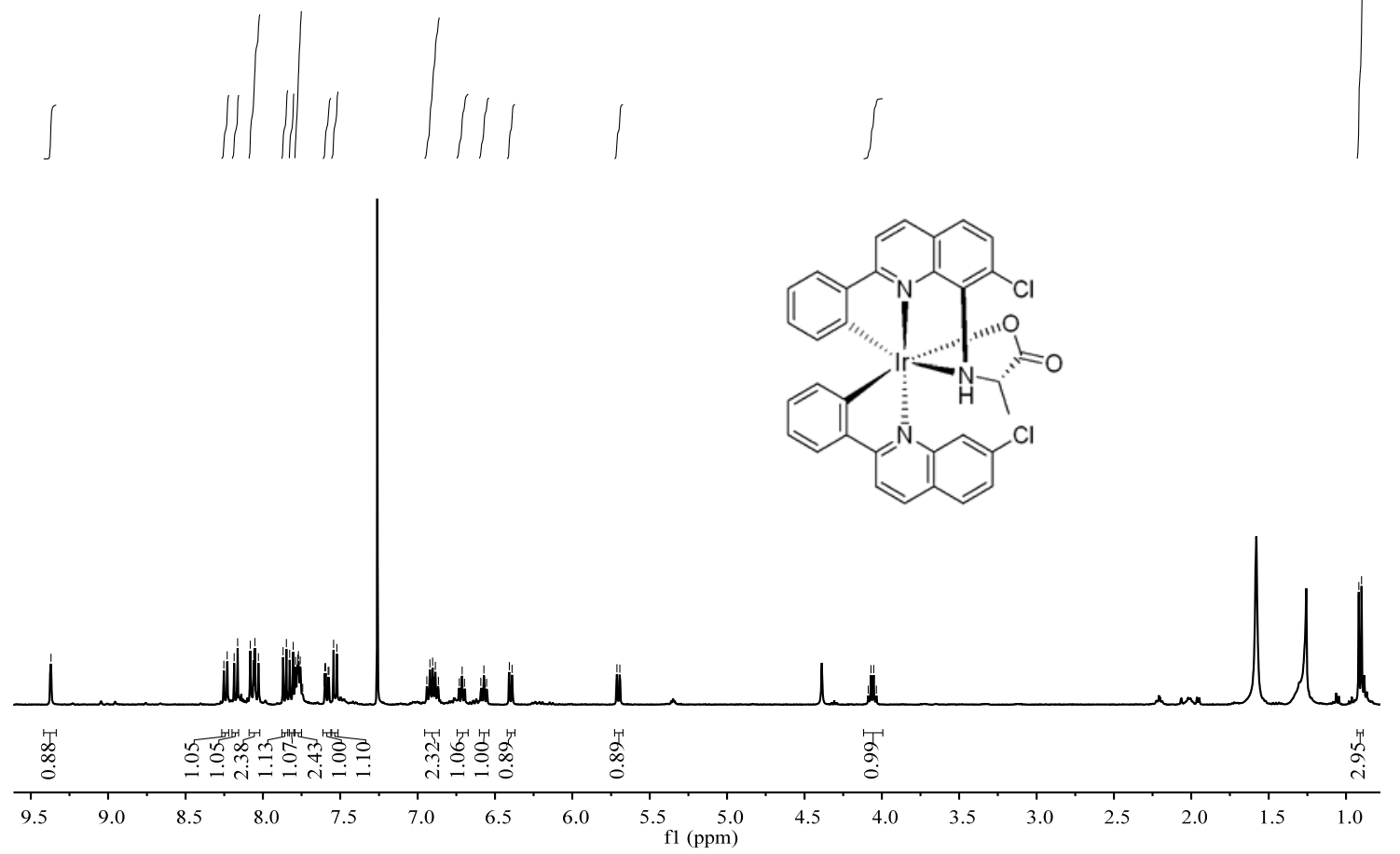

Figure S54. The ${ }^{1} \mathrm{H}$ NMR spectrum of $\Lambda$-[ $\left.\operatorname{Ir}(\mathrm{Cpq})(L-\mathrm{Cpqa})\right]$ in $\mathrm{CDCl}_{3}$. 


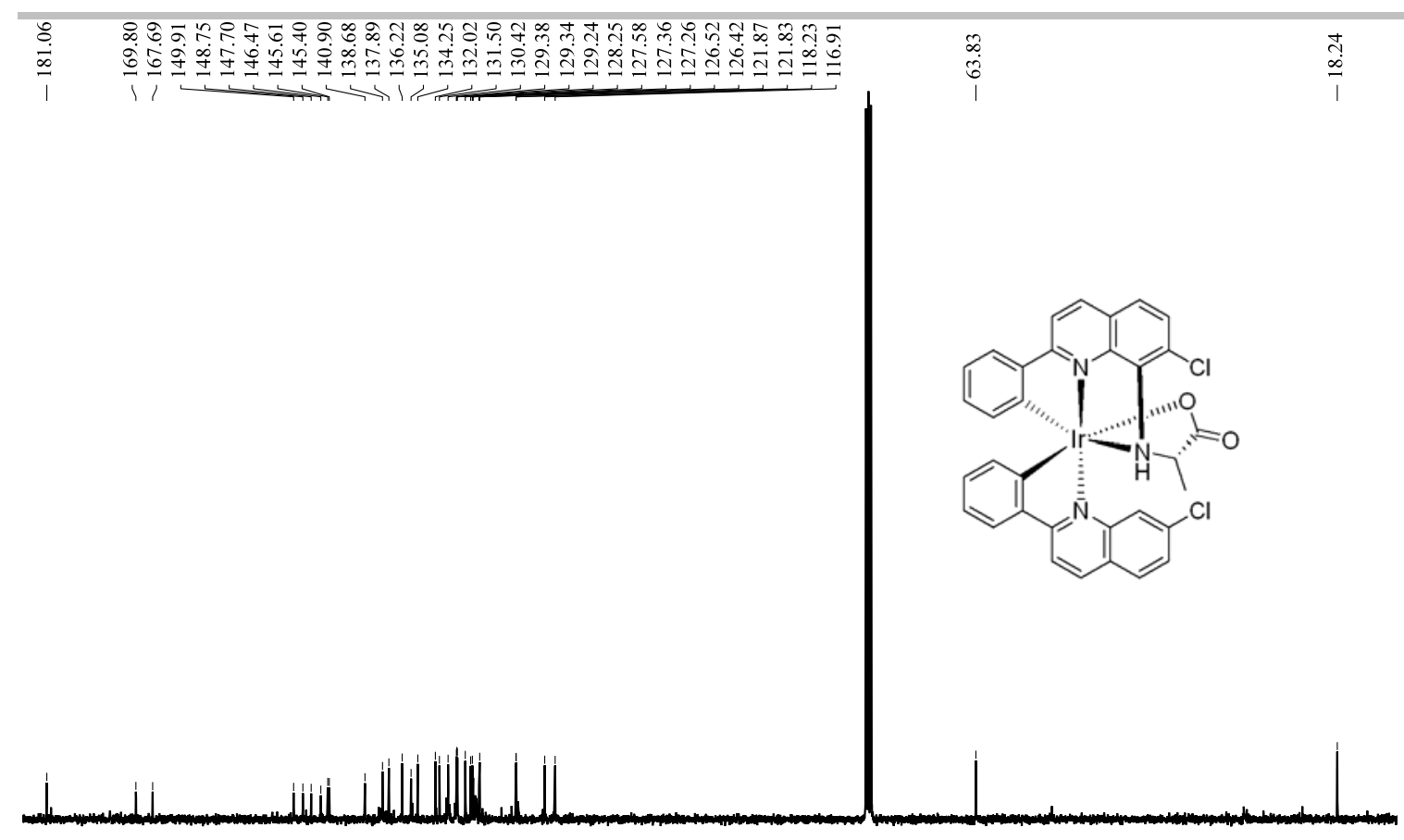

\begin{tabular}{lllllllllllllllll}
\hline 180 & 170 & 160 & 150 & 140 & 130 & 120 & 110 & $\underset{\mathrm{fl}(\mathrm{ppm})}{100}$ & 90 & 80 & 70 & 60 & 50 & 40 & 30 & 20
\end{tabular}

Figure S55. The ${ }^{13} \mathrm{C}$ NMR spectrum of $\Lambda$ - $[\operatorname{Ir}(\mathrm{Cpq})(L-\mathrm{Cpqa})]$ in $\mathrm{CDCl}_{3}$.

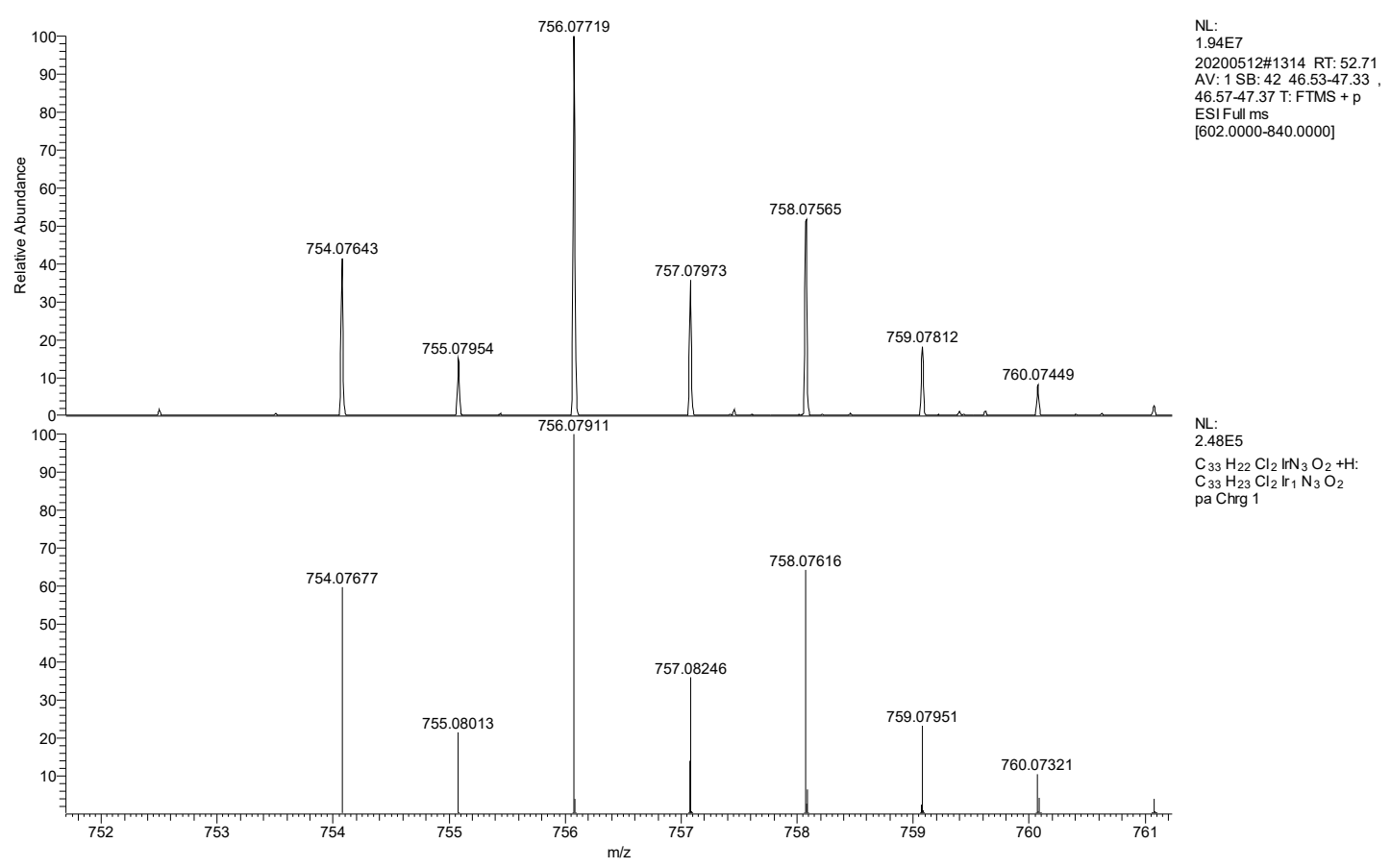

Figure S56. Comparison of HRMS of $\Lambda$-[Ir(Cpq) $(L-\mathrm{Cpqa})]$ at m/z $756.07719\left([\mathrm{M}+\mathrm{H}]^{+}\right)(\mathrm{up})$ and calculated for $\Lambda$-[ $\operatorname{Ir}(\mathrm{Cpq})(L-$ $\mathrm{Cpqa})]\left(\left[\mathrm{C}_{33} \mathrm{H}_{22} \mathrm{Cl}_{2} \mathrm{IrN}_{3} \mathrm{O}_{2}+\mathrm{H}\right]^{+}, 756.07911\right.$, down). 


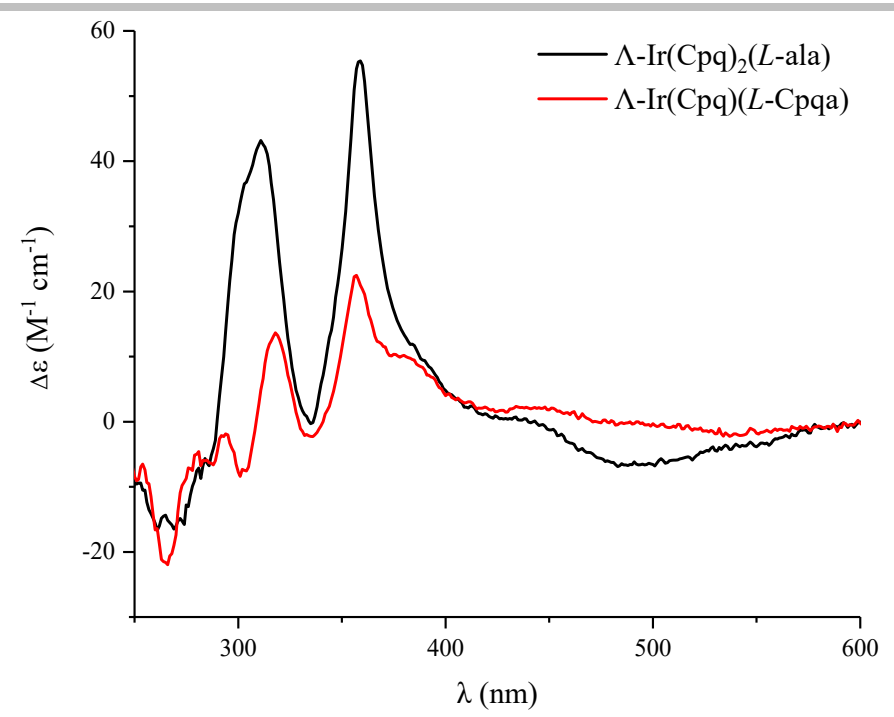

Figure S57. CD spectra of $\Lambda$-[ $\operatorname{Ir}(\mathrm{Cpq})_{2}(L$-ala $\left.)\right]$ and $\Lambda$-[Ir(Cpq) $\left.(L-\mathrm{Cpqa})\right]$ in $\mathrm{MeOH}(50 \mu \mathrm{M})$.

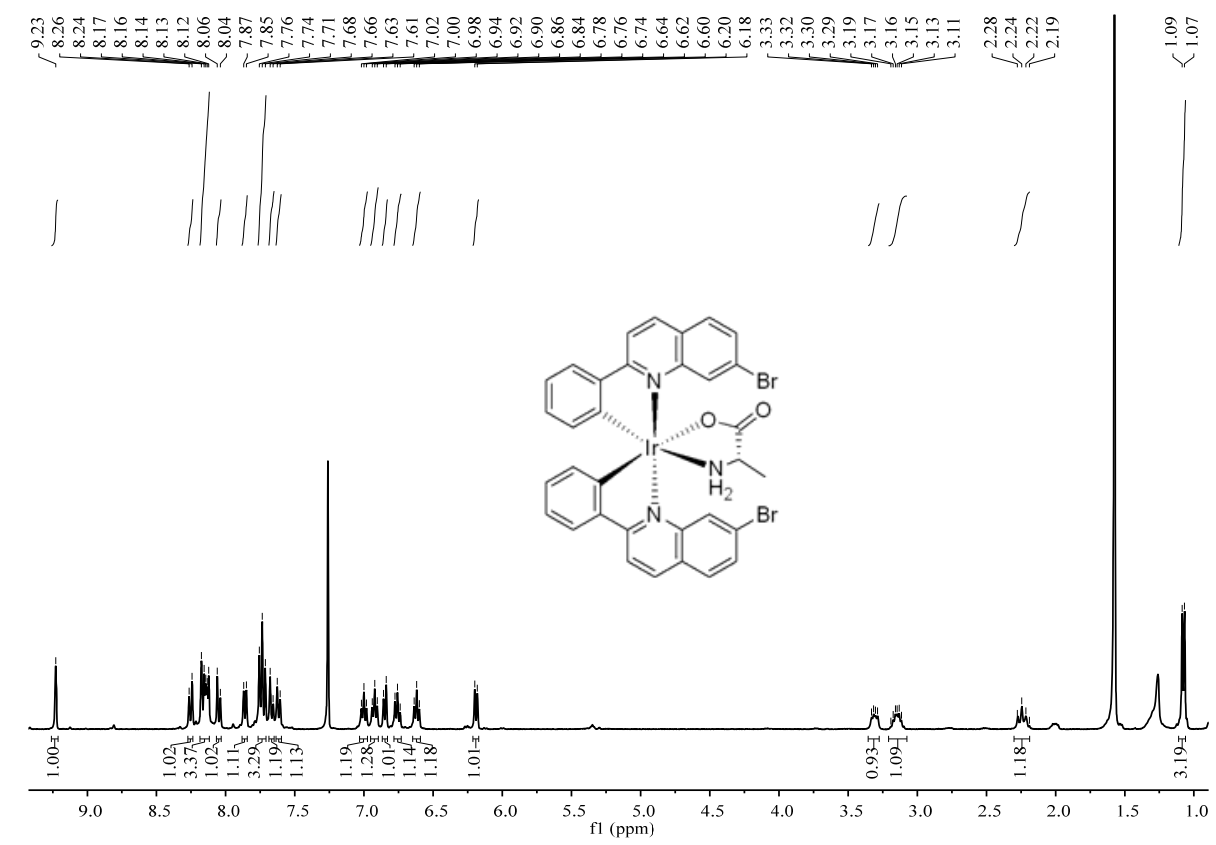

Figure S58. The ${ }^{1} \mathrm{H}$ NMR spectrum of $\Lambda$ - $\left[\operatorname{Ir}(\mathrm{Bpq})_{2}(L\right.$-ala $\left.)\right]$ in $\mathrm{CDCl}_{3}$. 

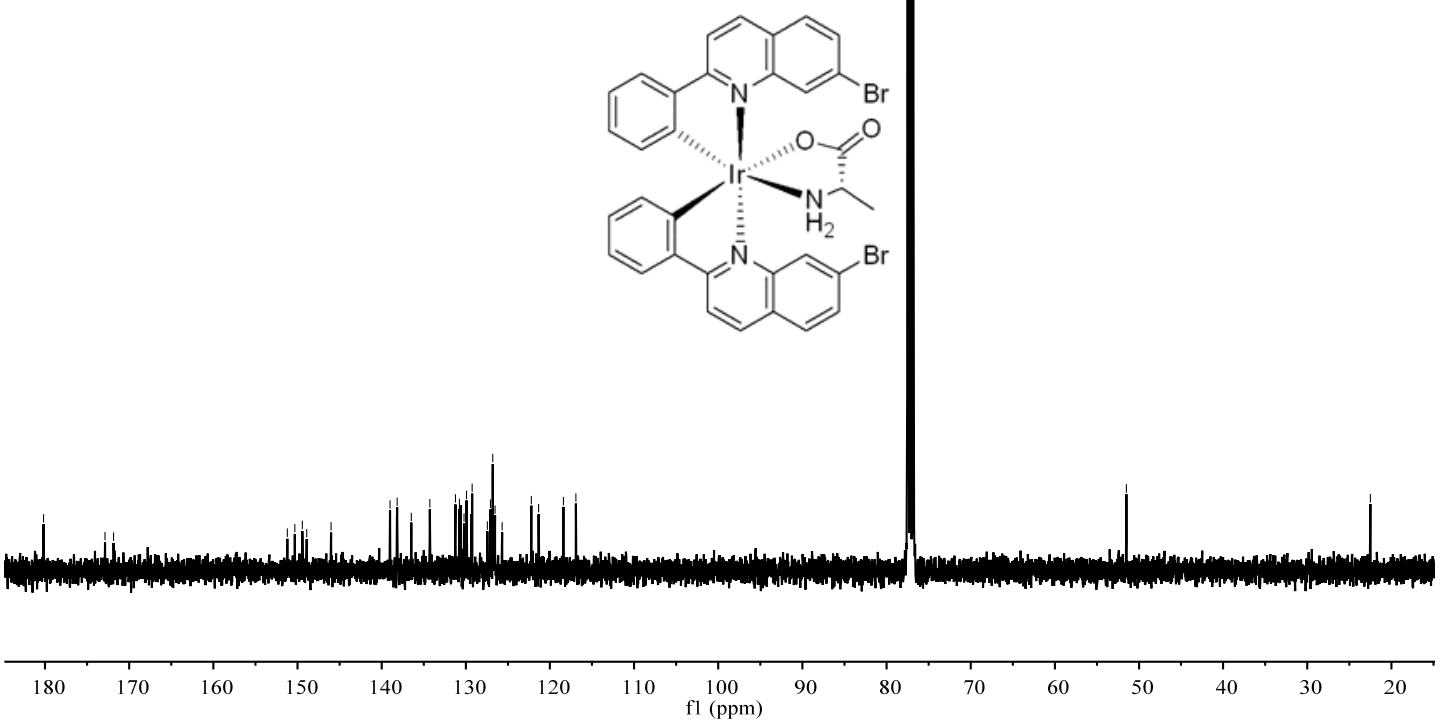

Figure S59. The ${ }^{13} \mathrm{C}$ NMR spectrum of $\Lambda$-[ $\operatorname{Ir}(\mathrm{Bpq})_{2}(L$-ala $\left.)\right]$ in $\mathrm{CDCl}_{3}$.

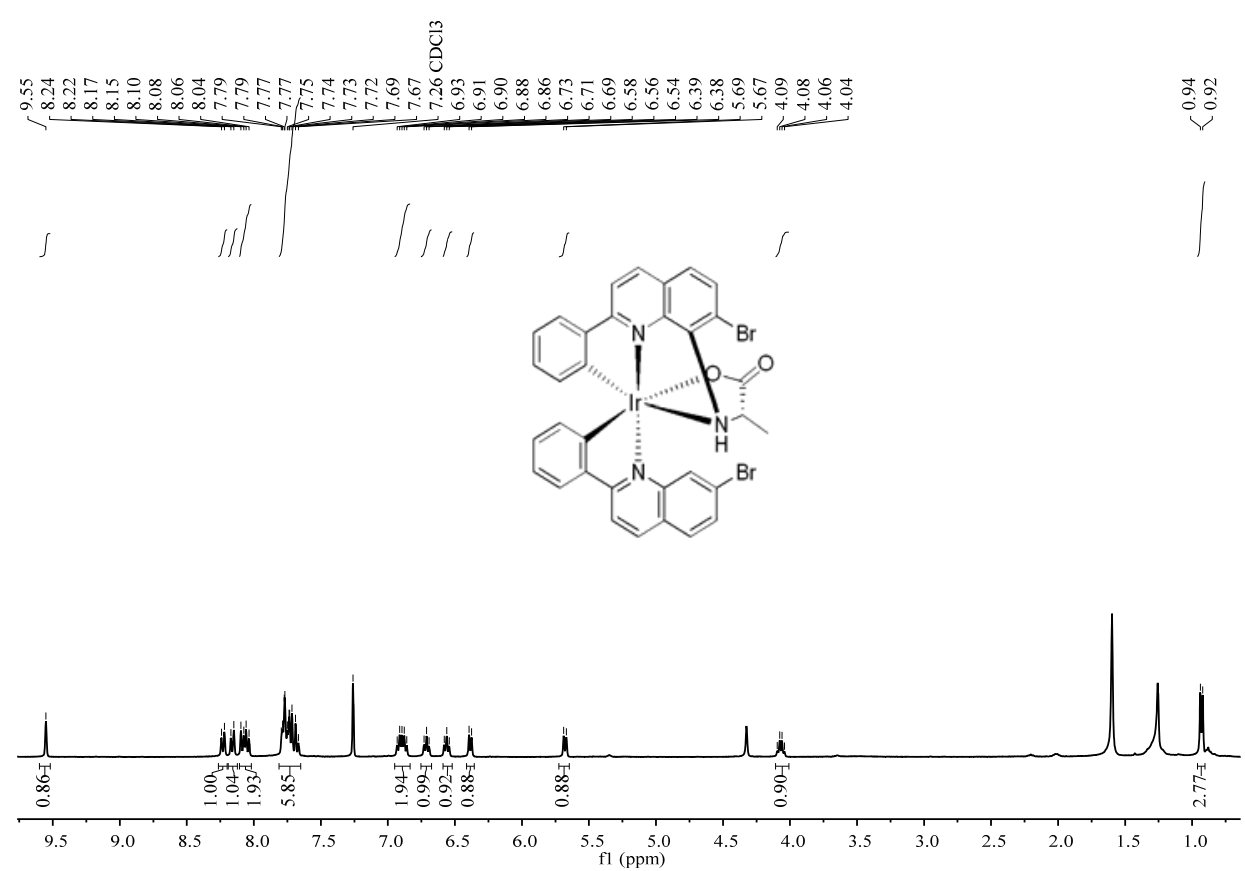

Figure S60. The ${ }^{1} \mathrm{H}$ NMR spectrum of $\Lambda$-[ $\operatorname{Ir}(\mathrm{Bpq})(L$-Bpqa) $]$ in $\mathrm{CDCl}_{3}$. 

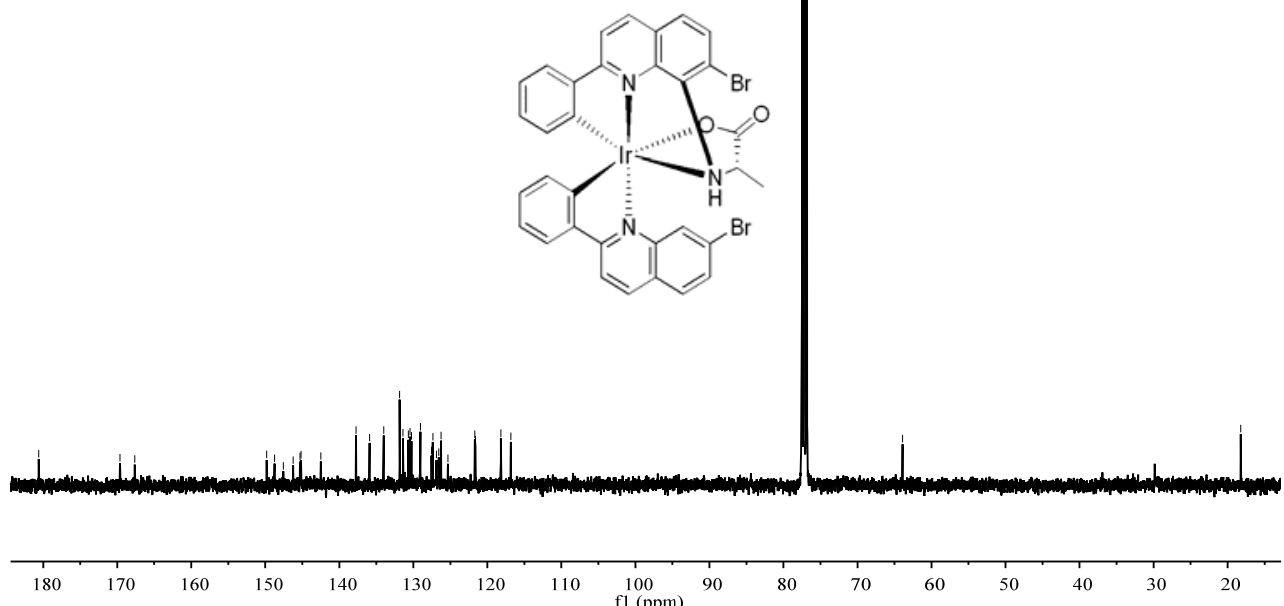

Figure S61. The ${ }^{13} \mathrm{C}$ NMR spectrum of $\Lambda$-[ $\operatorname{Ir}(\mathrm{Bpq})(L$-Bpqa $\left.)\right]$ in $\mathrm{CDCl}_{3}$.

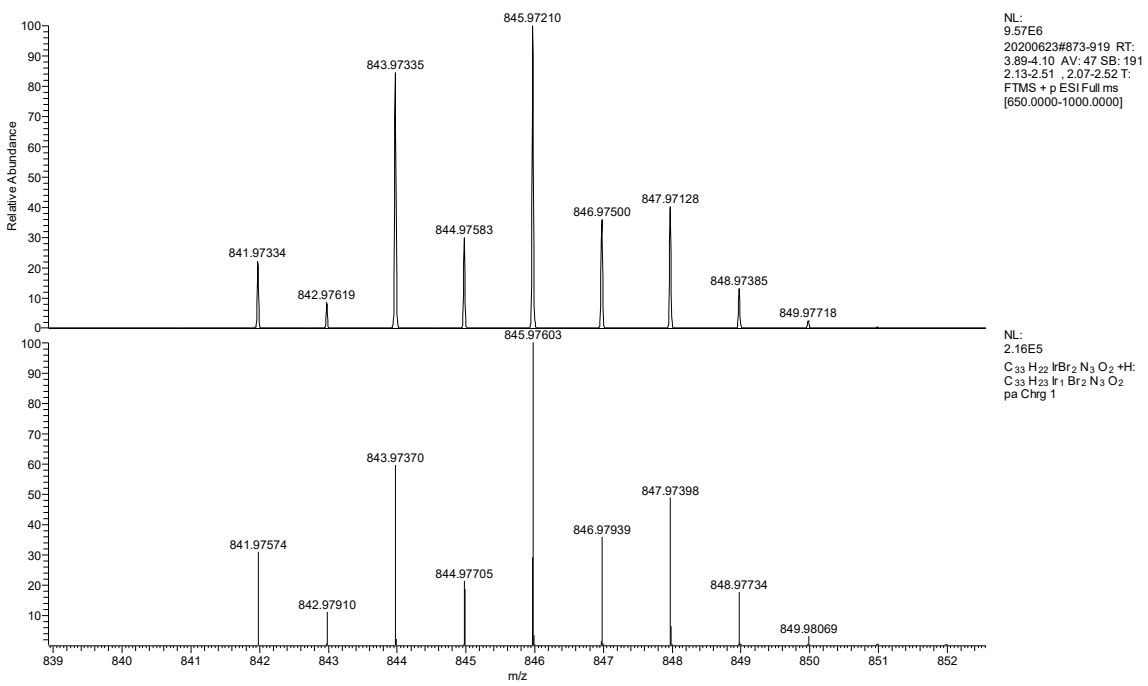

Figure S62. Comparison of HRMS of $\Lambda$-[Ir(Bpq) $(L-\mathrm{Bpqa})]$ at m/z $845.97210\left([\mathrm{M}+\mathrm{H}]^{+}\right)$(up) and calculated for $\Lambda$-[Ir(Bpq) $(L-$ Bpqa)] ([C $\left.\mathrm{C}_{33} \mathrm{H}_{22} \mathrm{Br}_{2} \mathrm{IrN}_{3} \mathrm{O}_{2}+\mathrm{H}\right]^{+}, 845.97603$, down).

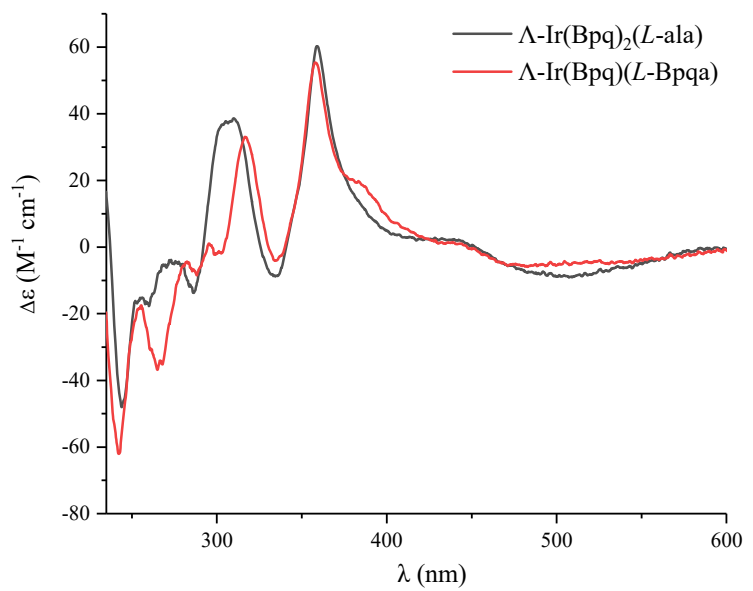

Figure S63. CD spectra of $\Lambda$-[Ir(Bpq $)_{2}(L$-ala $\left.)\right]$ and $\Lambda$-[Ir(Bpq) $(L$-Bpqa $\left.)\right]$ in $\mathrm{MeOH}(50 \mu \mathrm{M})$. 


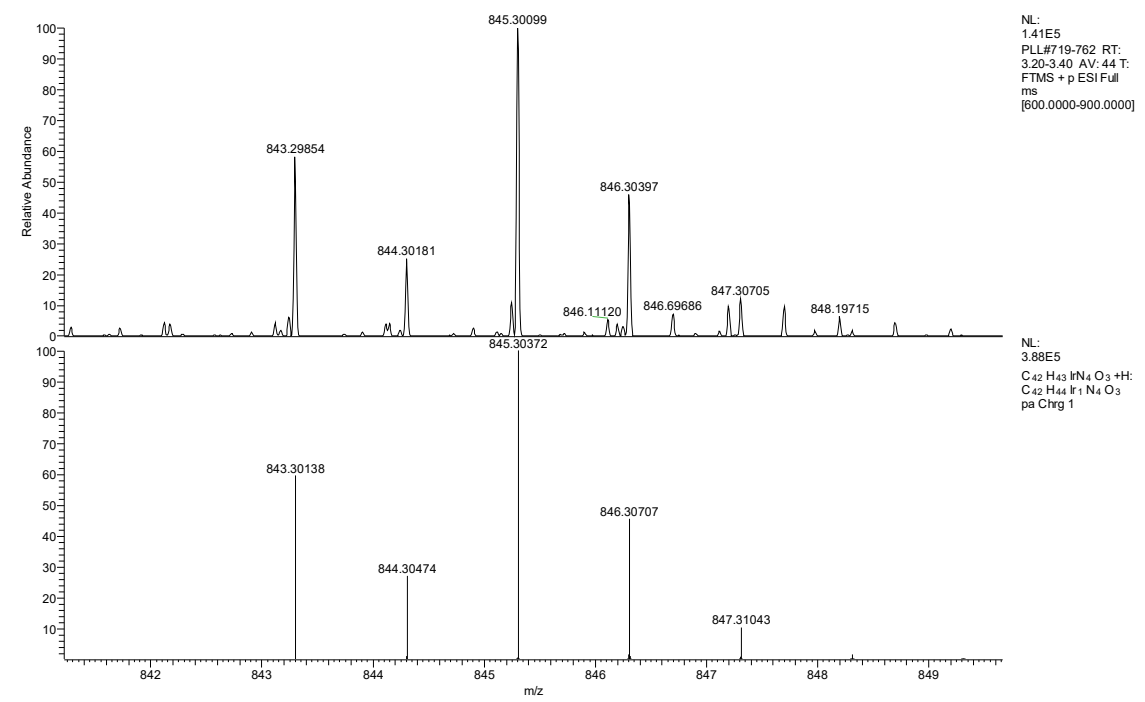

Figure S64. Comparison of HRMS of the product at m/z $845.30099\left([\mathrm{M}+\mathrm{H}]^{+}\right)$(up) and calculated for $\Lambda$ - $[\operatorname{Ir}(\mathrm{pq})(\mathrm{pq}-\mathrm{TEMPO})(L-$ ala) $]$ or $\Lambda$-[Ir(pq) $)_{2}(L$-ala-TEMPO) $]\left(\left[\mathrm{C}_{42} \mathrm{H}_{43} \mathrm{IrN}_{4} \mathrm{O}_{3}+\mathrm{H}\right]^{+}, 845.30372\right.$, down).

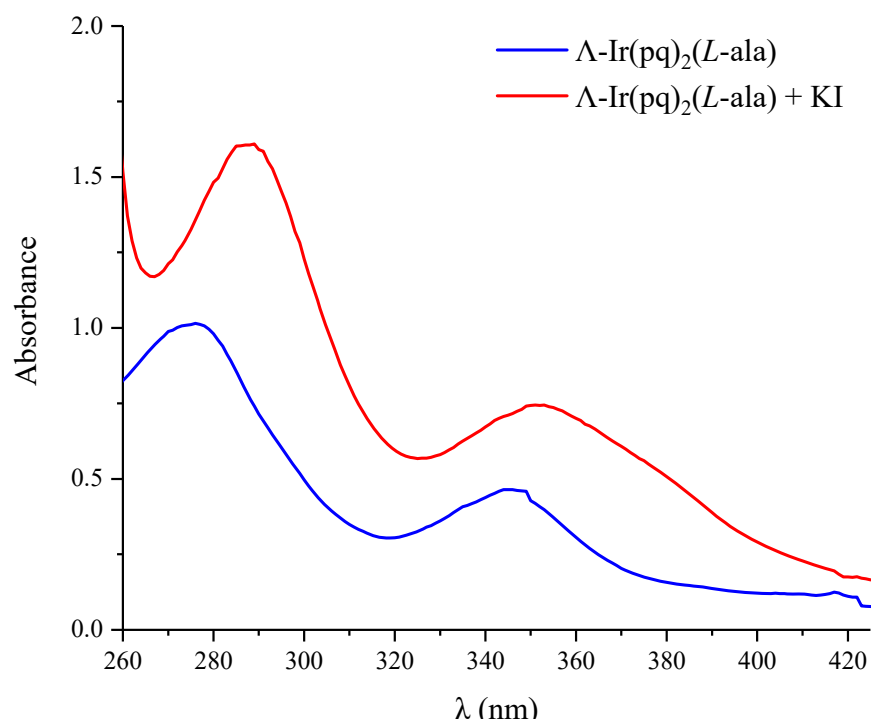

Figure S65. UV-vis absorption spectra of the tri-iodide formed by $\mathrm{H}_{2} \mathrm{O}_{2}$ oxidation. $(20 \mu \mathrm{M})$. 


\section{S7. Single-crystal X-ray crystallography}

The diffraction intensities were collected on a Bruker D8 Venture Photon III diffractometer with Helios Multilayer Optic-monochromated Ga Ka radiation $(\lambda=1.34138 \AA$ ). All of the data were corrected for absorption effect using the multi-scan technique. The structures were solved via direct methods (olex2.solve) ${ }^{[3]}$ and refined by iterative cycles of least-squares refinement on $\mathrm{F}^{2}$ followed by difference Fourier synthesis. ${ }^{[4]}$ All non-hydrogen atoms were refined anisotropically. All hydrogen atoms were included in the final structure factor calculation at idealized positions and were allowed to ride on the neighboring atoms. The crystal data and the details of data collection as well as refinement for the complex are summarized in Table S1. The selective bond lengths and angles are given in Table S2.

Table S1. Crystallographic Data for Ir(III) Complexes.

\begin{tabular}{|c|c|c|c|c|c|}
\hline Complex & $\Lambda-\operatorname{Ir}(\mathrm{pq})_{2}(L$-ala $)$ & $\Lambda-\operatorname{Ir}(\mathrm{pq})(L-\mathrm{pqa})$ & $\Delta$-Ir(pq)(D-pqv) & $\Delta-\operatorname{Ir}(\mathrm{pq})(D-\mathrm{pqs}) \cdot \mathrm{EtOH}$ & $\Delta-\operatorname{Ir}(\mathrm{pq})(D-\mathrm{pqp}) \cdot \mathrm{EtOH}$ \\
\hline Temperature/K & 150 & 293 & 150 & 293 & 150 \\
\hline Mol. formula & $\mathrm{C}_{33} \mathrm{H}_{26} \mathrm{IrN}_{3} \mathrm{O}_{2}$ & $\mathrm{C}_{33} \mathrm{H}_{24} \mathrm{IrN}_{3} \mathrm{O}_{2}$ & $\mathrm{C}_{35} \mathrm{H}_{28} \mathrm{IrN}_{3} \mathrm{O}_{2}$ & $\mathrm{C}_{35} \mathrm{H}_{30} \mathrm{IrN}_{3} \mathrm{O}_{4}$ & $\mathrm{C}_{37} \mathrm{H}_{32} \mathrm{IrN}_{3} \mathrm{O}_{3}$ \\
\hline$M_{\mathrm{r}}$ & 688.77 & 686.77 & 714.80 & 748.82 & 758.86 \\
\hline Crystal system & tetragonal & orthorhombic & orthorhombic & orthorhombic & monoclinic \\
\hline Space group & $P 4_{1}$ & $P 2{ }_{1}{ }_{1}{ }_{1}$ & $P 2{ }_{1}{ }_{1}{ }_{1}$ & $P 212121$ & $\mathrm{P} 2{ }_{1}$ \\
\hline$a / \AA$ & $13.2186(10)$ & $10.8178(3)$ & $8.6570(1)$ & $11.6496(2)$ & $11.1723(10)$ \\
\hline$b / \AA$ & $13.2186(10)$ & $12.0160(3)$ & $9.9134(2)$ & $15.0533(2)$ & $11.2535(10)$ \\
\hline$c / \AA$ & $17.8642(16)$ & $45.3299(10)$ & $33.1522(5)$ & $16.7306(2)$ & $12.3535(10)$ \\
\hline$V / \AA^{3}$ & $3121.4(5)$ & $5892.3(3)$ & $2845.13(8)$ & $2933.96(7)$ & $1459.37(2)$ \\
\hline$\beta /{ }^{\circ}$ & 90 & 90 & 90 & 90 & $110.015(10)$ \\
\hline Z & 4 & 8 & 4 & 4 & 2 \\
\hline$D_{c}\left(\mathrm{~g} \mathrm{~cm}^{-3}\right)$ & 1.466 & 1.548 & 1.669 & 1.695 & 1.727 \\
\hline$\mu\left(\mathrm{mm}^{-1}\right)$ & 5.647 & 9.030 & 9.376 & 9.175 & 9.206 \\
\hline${ }^{a} R_{1}[I>2 \sigma(\mathrm{I})]$ & 0.0464 & 0.0354 & 0.0411 & 0.0331 & 0.0418 \\
\hline${ }^{b} \omega R_{2}$ (all data) & 0.1424 & 0.0831 & 0.0984 & 0.0790 & 0.1095 \\
\hline GOF & 1.136 & 1.016 & 1.053 & 1.149 & 1.039 \\
\hline Flack parameter & $0.018(15)$ & $-0.018(7)$ & $-0.031(13)$ & $-0.031(7)$ & $-0.008(11)$ \\
\hline CCDC No & 2019907 & 2019908 & 2019906 & 2019905 & 2019904 \\
\hline
\end{tabular}

${ }^{\mathrm{a}} R_{1}=\Sigma|| F_{0}|-| F_{\mathrm{c}}|| \Sigma\left|F_{0}\right| .{ }^{b} \omega R_{2}=\left[\Sigma \omega\left(F_{0}^{2}-F_{\mathrm{c}}{ }^{2}\right)^{2} / \Sigma \omega\left(F_{0}^{2}\right)^{2}\right]^{1 / 2}$ 


\begin{tabular}{llllll} 
Table S2. Selective Bond Lengths $[\AA]$ and Angles $\left[{ }^{\circ}\right]$ \\
\hline Bond (Angle) & $\Lambda$-Ir(pq) $(L$-ala $)$ & $\Lambda$-Ir(pq)(L-pqa) & $\Delta$-Ir(pq)(D-pqv) & $\Delta$-Ir(pq)(D-pqs) & $\Delta$-Ir(pq)(D-pqp) \\
\hline Ir1-C1 & $2.003(15)$ & $1.995(9)$ & $2.005(9)$ & $1.983(8)$ & $1.980(6)$ \\
Ir1-C16 & $1.991(16)$ & $2.037(10)$ & $2.026(9)$ & $2.003(8)$ & $2.008(6)$ \\
Ir1-N1 & $2.084(13)$ & $2.083(7)$ & $2.094(8)$ & $2.113(6)$ & $2.0085(5)$ \\
Ir1-N2 & $2.082(11)$ & $1.965(7)$ & $1.993(8)$ & $1.989(6)$ & $1.962(5)$ \\
Ir1-N3 & $2.218(12)$ & $2.245(7)$ & $2.236(8)$ & $2.255(6)$ & $2.263(5)$ \\
Ir1-O1 & $2.167(9)$ & $2.176(6)$ & $2.187(7)$ & $2.187(6)$ & $2.188(5)$ \\
N3-C29 & & $1.452(11)$ & $1.488(13)$ & $1.458(11)$ & $1.461(8)$ \\
N3-C32 & $1.478(18)$ & $1.489(12)$ & $1.488(12)$ & $1.500(11)$ & $1.483(9)$ \\
O1-Ir1-N3 & $76.1(4)$ & $76.5(3)$ & $75.0(3)$ & $74.6(2)$ & $75.87(17)$ \\
N1-Ir1-C1 & $80.0(5)$ & $80.0(4)$ & $79.8(4)$ & $79.5(3)$ & $80.4(3)$ \\
N2-Ir1-C16 & $80.8(5)$ & $78.9(4)$ & $79.8(4)$ & $80.8(4)$ & $81.1(2)$ \\
N2-Ir-N3 & $97.2(4)$ & $81.4(3)$ & $80.9(4)$ & $79.8(3)$ & $79.89(19)$ \\
N3-Ir1-C16 & $165.3(6)$ & $159.4(4)$ & $159.8(4)$ & $160.1(3)$ & $160.6(2)$ \\
C1-Ir1-O1 & $175.8(6)$ & $170.1(3)$ & $171.0(3)$ & $173.0(3)$ & $169.3(2)$ \\
N2-Ir1-N1 & $174.3(5)$ & $173.6(3)$ & $173.9(3)$ & $175.2(3)$ & $171.9(2)$ \\
Ir1-N3-C32-C31 & $13.4(13)$ & $35.1(9)$ & $-37.4(8)$ & $-33.2(7)$ & $-39.2(6)$ \\
\hline
\end{tabular}




\section{S8. DFT calculations}

\section{S8.1 Computational details}

All the calculations were performed with the Gaussian 09 program. ${ }^{[5]}$ Geometry optimizations were carried out in the gas phase, by the density functional theory (DFT ${ }^{[6]}$ with the wB97XD functional, ${ }^{[7]}$ using def2-SVP basis sets. ${ }^{[8,9]}$ Frequency analysis calculations based on optimized structures were performed to characterize the structures to be minima (no imaginary frequency) or transition states (one imaginary frequency). Intrinsic reaction coordinate (IRC) calculations were taken to confirm the connection between two correct minima for a transition state. Based on wB97XD/def2-SVP optimized geometries, the energy results were further refined by calculating the single-point energy at the wB97XD/def2-TZVP level of theory. The bulky solvation effect of EtOH $(\varepsilon=24.9)$ was simulated by the SMD ${ }^{[10]}$ continuum solvent model at the wB97XD /def2-TZVP ${ }^{[8,9]}$ level of theory. The 3D optimized structures were displayed by using the CYLview visualization program. ${ }^{[11]}$ Cartesian coordinates of the optimized structures are given.

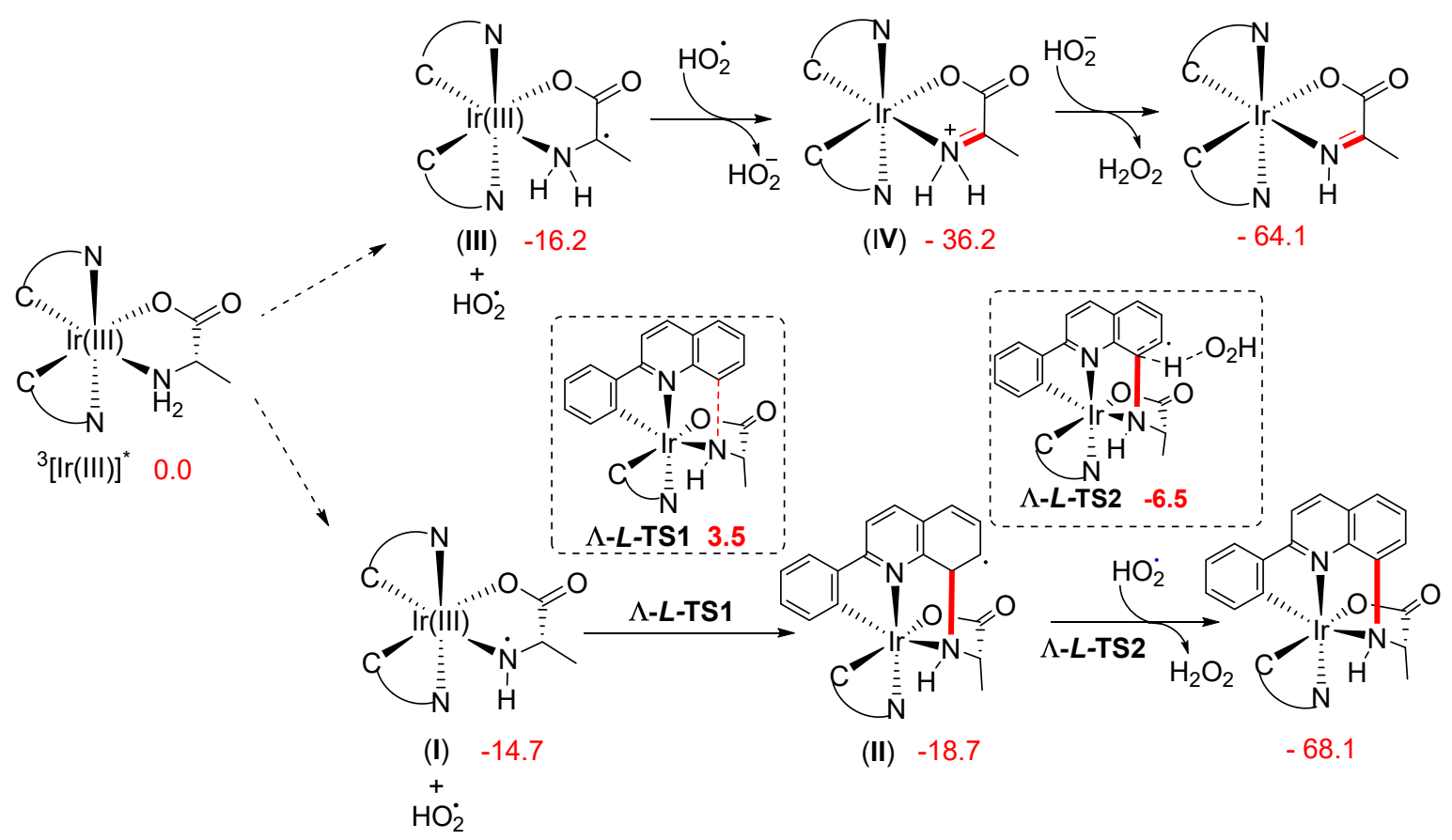

Figure S66. DFT free energy (in kcal/mol) calculated at wB97XD/def2-TZVP, SMD (EtOH)//wB97XD/def2-SVP level of theory. 


\section{S8.2 Cartesian Coordinates of Computed Structures}

$\mathrm{O}_{2}$

03

O $\quad 0.0000000 .0000000 .595378$

O $\quad 0.0000000 .000000-0.595378$

$\mathrm{OOH}^{\bullet}$

02

O $\quad 0.055116-0.5964600 .000000$

O $\quad 0.0551160 .7050950 .000000$

H $\quad-0.881864-0.8690760 .000000$

$\mathrm{OOH}^{-}$

$-11$

O $\quad 0.055785-0.6898770 .000000$

O $\quad 0.0557850 .7954860 .000000$

H $\quad-0.892558-0.8448730 .000000$

$\mathrm{HOOH}$

01

O $\quad 0.0000000 .7138380 .000000$
O $\quad 0.000000-0.7138380 .000000$

H $\quad 0.9510130 .8739420 .000000$

H $\quad-0.951013-0.8739420 .000000$

$\Lambda$-[Ir(pq) $)_{2}(D$-ala $\left.)\right]$

01

Ir $\quad-0.0404960 .0959750 .065573$

O $\quad-0.112275-1.950478-0.653730$

O $\quad 0.071594-3.296665-2.425702$

N $1.9968410 .528714-0.148262$

N $\quad-2.053477-0.3175880 .427136$

N $\quad-0.1218950 .253686-2.186005$

H $\quad-1.0888890 .312240-2.508053$

H $\quad 0.3416761 .091339-2.540661$

C $\quad-0.0566132 .0375660 .479950$

C $\quad-1.1274312 .8166980 .958904$

H $\quad-2.0593682 .3262501 .251823$

C $\quad-1.0224004 .1984171 .064915$

H $\quad-1.8702494 .7781001 .439397$

C $\quad 0.1592834 .8580550 .701379$ 
H $\quad 0.2340015 .9439450 .785342$

C $\quad 1.2426724 .1171590 .254346$

H $\quad 2.1696154 .634176-0.004135$

C $\quad 1.1427592 .7190040 .154990$

C $2.2617921 .843239-0.190329$

C $\quad 3.5642942 .318228-0.504703$

H $\quad 3.7383863 .391039-0.570168$

C $4.5779121 .432754-0.723465$

H $\quad 5.5806471 .783331-0.979654$

C $\quad 3.811849-2.674655-0.204881$

H $\quad 3.607152-3.734773-0.044753$

C $2.785421-1.769822-0.062556$

H $\quad 1.782810-2.1085680 .194365$

C $3.021619-0.388245-0.270786$

C $4.3409420 .040025-0.586160$

C $5.373443-0.919286-0.733958$

H $\quad 6.378376-0.571690-0.985342$

C $5.114988-2.256361-0.552588$

H $5.912303-2.993265-0.666503$

C $0.063840-0.4701991 .968712$

C $1.150031-0.3815072 .852449$

H 2.0470250 .1679932 .555947
C $\quad 1.104904-0.9932014 .101458$

H $1.962606-0.9132394 .774529$

C $\quad-0.025004-1.7133344 .507098$

H $\quad-0.049128-2.1950665 .486388$

C $-1.120028-1.8023463 .659394$

H $\quad-2.005984-2.3509733 .987090$

C $-1.082146-1.1761482 .404679$

C $\quad-2.217871-1.1196531 .486611$

C $\quad-3.438577-1.8225761 .684479$

H $\quad-3.523777-2.5123132 .522708$

C $-4.467115-1.6612700 .804950$

H $\quad-5.396091-2.2243270 .920102$

C $\quad-4.348829-0.719905-0.253738$

C $\quad-5.416029-0.431431-1.140145$

H $\quad-6.348405-0.991303-1.035709$

C $\quad-5.2904580 .546404-2.098098$

H $\quad-6.1176110 .766914-2.775064$

C $\quad-4.0940041 .292286-2.178764$

H $\quad-4.0150392 .109907-2.898458$

C $\quad-3.0324471 .017941-1.346770$

H $\quad-2.1380581 .640276-1.363098$

C $-3.118726-0.024753-0.388499$ 
C $\quad 0.100310-2.202762-1.896905$

C $0.489205-0.965978-2.755928$

C $\quad 0.171433-1.159298-4.227637$

H $\quad 0.545206-0.321507-4.837071$

H $\quad 0.628133-2.093725-4.576212$

H $\quad-0.915589-1.252501-4.381214$

H $\quad 1.579502-0.859158-2.625786$

$\Lambda$-[Ir(pq $\left.)_{2}(L-a l a)\right]$

01

Ir $\quad-0.0789320 .0975300 .051040$

O $\quad-0.101961-1.873232-0.831777$

O $\quad 0.272215-3.093502-2.662603$

N $1.9624670 .540441-0.064720$

N $\quad-2.097165-0.3628400 .326555$

N $\quad-0.1209350 .482393-2.184812$

H $\quad-1.0487140 .795248-2.461929$

H $\quad 0.5121181 .247395-2.420309$

C $\quad-0.1088431 .9969320 .630181$

C $\quad-1.1907322 .7353401 .147032$

H $\quad-2.1290332 .2236981 .376701$
C -1.0891314 .1038011 .368910$

H $\quad-1.9458614 .6515361 .770268$

C $\quad 0.1005754 .7889441 .089040$

H $\quad 0.1727225 .8644401 .261878$

C $\quad 1.1951674 .0853010 .609827$

H 2.1279734 .6202770 .417698

C $\quad 1.0987212 .7002930 .393932$

C 2.2282841 .8531530 .010740

C $3.5399322 .349907-0.219445$

H $3.7163863 .423741-0.185620$

C $\quad 4.5598811 .483043-0.479331$

H $\quad 5.5702181 .851270-0.673146$

C $3.777944-2.651404-0.347317$

H $\quad 3.566815-3.720819-0.292135$

C $2.748792-1.760346-0.149691$

H $\quad 1.739021-2.1206850 .041249$

C $2.990631-0.366490-0.228083$

C $4.3183590 .084610-0.468016$

C $5.354157-0.860410-0.673168$

H $\quad 6.365396-0.494013-0.865470$

C $5.090016-2.207572-0.621314$

H $\quad 5.889367-2.933557-0.781768$ 

C $-0.015084-0.6265011 .900906$
C $\quad-3.0714421 .123290-1.326436$
C $1.044964-0.5941472 .819176$
H $\quad-2.2048671 .778703-1.237911$
H $1.940616-0.0100412 .593341$
C $-3.147775-0.018427-0.487638$
C $0.975394-1.3056914 .012655$
C $\quad 0.122121-2.033263-2.086451$
H $\quad 1.811938-1.2676524 .715303$
C $0.232779-0.742615-2.945008$
C $\quad-0.153123-2.0729264 .325935$
H $\quad-0.493944-0.875962-3.761856$
H $\quad-0.196707-2.6309425 .263234$
C $\quad 1.623944-0.635212-3.555966$
C $\quad-1.222183-2.1101653 .442069$
H $\quad 1.6792340 .185789-4.288540$
H $\quad-2.107753-2.6961293 .698926$
H $\quad 1.857303-1.579380-4.063990$
C $-1.160416-1.3826132 .244075$
H $\quad 2.387739-0.468544-2.781450$
C $\quad-2.270451-1.2651361 .300980$
C $\quad-3.475316-2.0159381 .391832$
${ }^{3}[\operatorname{Ir}(I I I)]$
H $\quad-3.565273-2.7832322 .159106$
03
C $\quad-4.478938-1.8030760 .494812$
Ir $\quad 0.0345920 .003991-0.161512$
H $\quad-5.391477-2.4031280 .521856$
O $\quad 0.1719121 .0209391 .671384$
C $\quad-4.357416-0.760694-0.464377$
O $\quad-0.2246581 .0624933 .862537$
C $\quad-5.405460-0.417172-1.353708$
N $-1.978656-0.417037-0.251864$
H $\quad-6.320086-1.014811-1.339727$
N $\quad 2.1092890 .500618-0.157666$
C $\quad-5.2857230 .658288-2.201438$
N $\quad-0.109753-1.5919301 .490644$
H $\quad-6.0988600 .920970-2.880293$
H $\quad 0.788078-1.9925171 .761563$
C $\quad-4.1165201 .449443-2.160863$
H $\quad-0.687175-2.3592981 .143342$
H $\quad-4.0477372 .344665-2.782739$
C $0.118313-1.428204-1.541527$ 

C $1.235008-1.817105-2.291831$
C $\quad-5.0965061 .6140891 .720965$
H $\quad 2.182659-1.286732-2.168282$
H $\quad-5.9003762 .1312422 .248594$
C $\quad 1.154798-2.875507-3.195963$
C $\quad 0.0105351 .595964-1.329740$
H $\quad 2.030533-3.174583-3.775377$
C $\quad-1.0916762 .077305-2.051432$
C $\quad-0.062270-3.547399-3.354421$
H $\quad-2.0205711 .504493-2.063020$
H $\quad-0.134508-4.375320-4.064077$
C $\quad-1.0018863 .281821-2.742295$
C $-1.191271-3.166870-2.636676$
H $\quad-1.8627273 .649216-3.305234$
H $\quad-2.135381-3.686349-2.812302$
C $\quad 0.1781954 .031167-2.712005$
C $-1.125623-2.104525-1.716858$
H $\quad 0.2377814 .979503-3.249618$
C $\quad-2.245287-1.591046-0.952777$
C $\quad 1.2803983 .573869-1.996010$
C $-3.520342-2.170228-0.901951$
H $\quad 2.1959314 .168525-1.987904$
H $\quad-3.694379-3.109123-1.430060$
C $\quad 1.2030392 .356310-1.312590$
C $\quad-4.540793-1.576289-0.196120$
C $2.3173241 .751195-0.574620$
H $\quad-5.529436-2.034884-0.139625$
C $3.5380582 .423863-0.306056$
C $\quad-3.8249742 .1851481 .630078$
H $\quad 3.6624883 .457338-0.625136$
H $\quad-3.6247303 .1566012 .086617$
C $\quad 4.5214581 .7848950 .391779$
C $\quad-2.7969101 .5293930 .963585$
H $\quad 5.4505242 .2996860 .646733$
H $\quad-1.8127271 .9918490 .912295$
C $\quad 4.3514120 .4266660 .767042$
C $\quad-3.0039260 .2654760 .376983$
C $5.365846-0.3142181 .424752$
C $\quad-4.310688-0.3096900 .446033$
H $\quad 6.2935220 .1948151 .695937$
C $\quad-5.3282670 .3811921 .125479$
C $5.192692-1.6489471 .698774$
H $\quad-6.319371-0.0773721 .176127$
H $\quad 5.978289-2.2152322 .201939$ 

C $5.415390-0.681545-0.642439$
C $\quad-5.2767830 .686301-2.255536$
H $\quad 6.414617-0.271229-0.811548$
H $\quad-6.0867130 .936425-2.945676$
C $5.202897-2.039398-0.624200$
C $\quad-4.1366371 .480787-2.185877$
H $\quad 6.032836-2.730311-0.788895$
H $\quad-4.0516382 .382467-2.798636$
C $\quad-0.020063-0.6957171 .893596$
C $\quad-3.0937791 .145817-1.312465$
C $1.046951-0.6757792 .798511$
H $\quad-2.2440021 .820341-1.201762$
H $\quad 1.950867-0.1088592 .555472$
C $-3.147779-0.010516-0.504172$
C $0.988092-1.3774534 .005213$
C $\quad 0.295252-2.011092-2.155482$
H $\quad 1.836833-1.3517624 .695091$
C $0.268185-0.692753-2.981241$
C $\quad-0.154403-2.1113944 .329766$
H $\quad-0.403538-0.894893-3.832115$
H $\quad-0.206745-2.6619335 .273199$
C $1.655238-0.390529-3.532941$
C $\quad-1.238348-2.1279443 .455524$
H $1.6303860 .444411-4.252957$
H $\quad-2.139294-2.6837963 .729202$
H $\quad 2.033532-1.287291-4.040185$
C $\quad-1.187718-1.4218402 .244559$
H $\quad 2.357520-0.132101-2.726472$
C $\quad-2.294216-1.3271291 .292660$
C $\quad-3.469857-2.0617221 .347870$
$[\operatorname{lr}(\mathrm{IV})]$
H $\quad-3.582998-2.8226662 .122084$
12
C $\quad-4.488753-1.8474350 .423795$
Ir $\quad-0.1016280 .0435890 .130469$
H $\quad-5.402483-2.4458460 .439793$
O $-0.099246-1.389359-1.370700$
C $-4.366298-0.776396-0.510477$
O $\quad 0.806647-2.030348-3.302503$
C $-5.389768-0.418896-1.409871$
N 1.9450280 .4979210 .175748
H $\quad-6.297509-1.029726-1.424792$
N $\quad-2.141004-0.4605550 .182776$ 

N $\quad-0.0300031 .185708-1.882329$
C $2.974416-0.318198-0.244155$
H $\quad-0.9627111 .523554-2.111236$
C $4.2902580 .214397-0.375925$
H $\quad 0.5570782 .016942-1.801931$
C $5.336504-0.612245-0.859112$
C $\quad-0.1935221 .7527101 .141084$
H $\quad 6.335383-0.184244-0.966535$
C $\quad-1.3136872 .3230621 .764375$
C $5.092937-1.924042-1.180669$
H $\quad-2.2649911 .7869131 .775069$
H $\quad 5.896879-2.558160-1.557588$
C $\quad-1.2191623 .5674702 .381761$
C $\quad-0.075534-1.2222021 .638101$
H $\quad-2.0931983 .9990822 .873597$
C $\quad 0.999132-1.4925402 .499447$
C $\quad-0.0092214 .2661652 .376330$
H $1.912291-0.8971252 .443800$
H $\quad 0.0596065 .2432692 .857586$
C $\quad 0.902733-2.5190783 .433622$
C $\quad 1.1178743 .7149081 .771492$
H $\quad 1.735665-2.7195064 .110396$
H $\quad 2.0589364 .2663191 .802557$
C $\quad-0.253617-3.3015073 .506355$
C $\quad 1.0336462 .4612161 .158903$
H $\quad-0.317719-4.1104784 .236093$
C $\quad 2.1889361 .7506100 .585371$
C $\quad-1.329572-3.0516912 .656814$
C $\quad 3.4801022 .3158680 .486554$
H $\quad-2.226289-3.6682792 .737156$
H $\quad 3.6446943 .3460400 .797056$
C $\quad-1.250069-2.0089181 .732739$
C $4.5092831 .563986-0.011996$
C $\quad-2.345789-1.6060430 .843772$
H $\quad 5.5084131 .992599-0.117772$
C $\quad-3.541018-2.3461260 .683032$
C $3.796122-2.458559-1.010548$
H $\quad-3.661093-3.2944561 .203750$
H $\quad 3.606028-3.505217-1.253120$
C $-4.508781-1.884675-0.164143$
C $\quad 2.758332-1.684768-0.547150$
H $\quad-5.417968-2.463651-0.339734$
H $\quad 1.769265-2.122190-0.432873$
C $-4.352933-0.624346-0.795385$ 

C $\quad-5.365059-0.057672-1.612040$
N $\quad 2.0520750 .361639-0.399198$
H $\quad-6.270817-0.637519-1.801660$
N $\quad-2.019272-0.0494660 .514617$
C $\quad-5.2195971 .203140-2.136326$
N $\quad-0.028271-0.823194-1.875755$
H $\quad-6.0030691 .636050-2.759917$
C $\quad-0.0116421 .987417-0.568404$
C $\quad-4.0651801 .956714-1.828002$
C $-1.1104912 .863650-0.564259$
H $\quad-3.9811042 .984501-2.187087$
H $\quad-2.0715732 .526955-0.169138$
C $\quad-3.0557941 .423949-1.059099$
C $\quad-0.9954264 .159158-1.055812$
H $\quad-2.2133062 .045898-0.760707$
H $\quad-1.8646054 .821903-1.042595$
C $\quad-3.1531710 .100101-0.559295$
C $\quad 0.2217274 .625223-1.566617$
C $0.409684-1.175939-2.552262$
H $\quad 0.3043405 .641513-1.956609$
C $\quad 0.4594650 .309009-2.974409$
C $\quad 1.3278413 .788390-1.564445$
H $\quad-0.2330060 .385257-3.827596$
H $\quad 2.2772074 .163836-1.952369$
C $\quad 1.8516640 .700488-3.447636$
C $\quad 1.2162852 .482266-1.061382$
H $\quad 1.8497911 .700255-3.906543$
C $\quad 2.3359171 .549075-0.951883$
H $\quad 2.186676-0.028409-4.196226$
C $\quad 3.6560451 .868622-1.370263$
H $\quad 2.5762980 .690878-2.619951$
H $\quad 3.8472352 .834455-1.834312$
C $4.6635420 .967950-1.193912$
Intermediate I
H $\quad 5.6807651 .194988-1.522017$
02
C $3.815163-2.6553920 .768643$
Ir $\quad-0.0001050 .068561-0.033504$
H $\quad 3.587111-3.5836071 .296099$
O $\quad-0.120649-2.0590200 .426175$
C $2.799093-1.7558570 .539126$
O $\quad-0.322408-4.082894-0.502508$
H $\quad 1.785520-1.9739280 .876258$ 

C $3.062683-0.546315-0.148999$
C $\quad-5.461231-0.691681-0.731350$
C $4.397826-0.272046-0.558503$
H $\quad-6.431618-0.989477-0.327419$
C $5.419168-1.222637-0.311603$
C $\quad-5.301635-0.452172-2.074151$
H $\quad 6.435824-0.995893-0.641502$
H $\quad-6.143423-0.563050-2.760035$
C $\quad 5.134618-2.3998350 .336780$
C $-4.040951-0.044146-2.563571$
H $\quad 5.922941-3.1311110 .525287$
H $\quad-3.9184230 .171968-3.626830$
C $\quad 0.1552850 .4935741 .943000$
C $\quad-2.9604240 .088345-1.723301$
C $\quad 1.2803780 .9007582 .670862$
H $\quad-1.9920240 .406334-2.105480$
H $\quad 2.2133431 .1296142 .149772$
C $-3.092843-0.179544-0.337105$
C $\quad 1.2343711 .0151794 .058373$
C $\quad-0.286088-2.873301-0.552292$
H $\quad 2.1242011 .3363494 .605918$
C $\quad-0.492192-2.172978-1.952482$
C $\quad 0.0602730 .7220644 .757630$
H $\quad-1.598454-2.153462-2.042150$
H $\quad 0.0288550 .8121655 .845231$
C $\quad 0.095371-2.986513-3.092220$
C $\quad-1.0721210 .3225164 .059667$
H $\quad-0.161899-2.549681-4.069369$
H $\quad-1.9880170 .1070514 .613873$
H $\quad-0.291512-4.011974-3.042583$
C $\quad-1.0270820 .2134072 .662492$
H $\quad 1.191827-3.039860-3.002477$
C $\quad-2.192619-0.1266301 .839801$
H $\quad 0.659814-0.595705-2.596207$
C $\quad-3.452832-0.4871982 .387710$
H $\quad-3.553693-0.5921323 .466264$
Intermediate II
C $\quad-4.513345-0.7221011 .564037$
02
H $\quad-5.480848-1.0241881 .971707$
Ir $\quad-0.036168-0.137614-0.009444$
C $\quad-4.371676-0.5442180 .163356$
O $\quad-0.2744431 .463454-1.451368$ 

O $\quad-0.8045093 .614467-1.749290$
H $\quad-2.8740852 .8912692 .723120$
N $\quad-0.8861781 .5779271 .228130$
C $\quad-4.5357082 .2984161 .504125$
H $\quad-0.4418031 .6474792 .142229$
H $\quad-5.1980683 .0471401 .944332$
N $\quad 2.025347-0.0085830 .445996$
C $\quad 1.465927-2.1990173 .365017$
N $-1.977396-0.341321-0.402351$
H $\quad 2.417471-2.1999443 .901223$
C $\quad 0.417015-2.9642833 .855090$
C $\quad 0.065883-1.4280211 .498597$
H $\quad 0.545848-3.5520634 .765914$
C $\quad-4.1880740 .517327-0.163135$
C $3.688561-0.5990562 .081698$
C $\quad-2.8459480 .4363340 .237864$
H $\quad 3.924191-1.1197013 .008324$
C $2.365158-0.6576301 .568150$
C $-3.651738-1.133630-1.868471$
C 4.3280910 .7448670 .191225
H $\quad-3.954299-1.762377-2.705696$
C $\quad 1.298607-1.4424702 .194455$
C $\quad-0.800766-2.9832323 .165655$
C $\quad-2.2982351 .1612181 .444696$
H $\quad-1.623558-3.5987383 .538852$
C $0.086833-1.578808-1.389072$
C $\quad 2.6666521 .262171-1.524694$
C $\quad 4.6487170 .1021701 .414527$
H $\quad 1.6436761 .207715-1.899407$
H 5.6681830 .1639841 .802882
C $\quad 5.2980311 .448952-0.564998$
C $2.9891430 .658740-0.283157$
H $\quad 6.3186791 .509529-0.179582$
C $-2.311509-1.132813-1.445705$
C $\quad-1.284771-2.822329-3.014290$
C $\quad-0.6073392 .639133-1.052115$
H $\quad-2.255784-3.035376-3.468554$
C $\quad-1.173197-1.875919-1.986624$
C $\quad-5.0490921 .4483350 .503685$
C $\quad 1.085297-3.223950-2.876444$
H $\quad-6.0902501 .5338150 .188261$
H $\quad 1.973749-3.756796-3.225149$
C $\quad-3.2364892 .2214511 .939255$
C $\quad-0.971355-2.2313812 .007594$ 
H $\quad-1.929937-2.2733911 .482226$

C $\quad-0.6816102 .8424140 .486613$

H $\quad-1.5257413 .5261940 .669995$

C $\quad 0.6094803 .5032800 .955430$

H $\quad 0.5678453 .7519192 .028529$

H $\quad 0.7628864 .4306590 .389187$

H 1.4749012 .8454690 .779586

C $1.204369-2.280825-1.858313$

H $\quad 2.185922-2.085301-1.419289$

C $\quad-0.156420-3.497081-3.460246$

H $\quad-0.238922-4.237063-4.258604$

C $\quad 3.6342991 .933121-2.236957$

H $\quad 3.3640182 .394078-3.188984$

C $-4.570596-0.309820-1.245036$

H $\quad-5.604999-0.283570-1.595408$

C $4.9590782 .037579-1.758920$

H $\quad 5.7081862 .579618-2.339374$

H $\quad-2.2237000 .3807042 .232380$

Intermediate III

02

Ir $\quad-0.1081420 .0583470 .073518$
O $\quad-0.053777-1.810570-1.038219$

O $\quad 0.955411-2.774329-2.795631$

N 1.9302750 .5555560 .100008

N $\quad-2.130312-0.4271130 .166505$

N $\quad 0.0561830 .637928-2.154034$

H $\quad-0.8967620 .666533-2.525803$

H $\quad 0.4787851 .549411-2.333233$

C $\quad-0.2100101 .8862100 .845784$

C $\quad-1.3331722 .5426281 .383175$

H $\quad-2.2689061 .9915671 .509004$

C $\quad-1.2763993 .8814331 .753732$

H $\quad-2.1641554 .3661742 .168423$

C $\quad-0.0927874 .6160801 .605554$

H $\quad-0.0559745 .6682781 .894468$

C $\quad 1.0392303 .9909601 .104391$

H 1.9659054 .5622421 .013210

C $\quad 0.9874752 .6356300 .738851$

C $\quad 2.1512451 .8626230 .303095$

C $\quad 3.4450992 .4226680 .126697$

H 3.5864683 .4929360 .268010

C $4.4902991 .621682-0.230009$

H $\quad 5.4853532 .042453-0.393855$ 


\begin{tabular}{|c|c|c|c|}
\hline C & $3.845598-2.530584-0.491985$ & $\mathrm{H}$ & $-3.758859-2.9384611 .718880$ \\
\hline $\mathrm{H}$ & $3.668214-3.606067-0.540611$ & C & $-4.551072-1.8078440 .087424$ \\
\hline C & $2.794418-1.697949-0.187573$ & $\mathrm{H}$ & $-5.480556-2.3779270 .020739$ \\
\hline $\mathrm{H}$ & $1.803754-2.1084160 .001031$ & C & $-4.347185-0.702402-0.783431$ \\
\hline C & $2.986899-0.295520-0.150693$ & C & $-5.330598-0.262736-1.704116$ \\
\hline C & $4.2951370 .222449-0.361384$ & $\mathrm{H}$ & $-6.261245-0.829284-1.785768$ \\
\hline C & $5.356889-0.662859-0.673092$ & C & $-5.1295610 .864828-2.464432$ \\
\hline $\mathrm{H}$ & $6.352339-0.246040-0.843996$ & $\mathrm{H}$ & $-5.8934741 .200141-3.167982$ \\
\hline C & $5.135281-2.016500-0.749874$ & C & $-3.9408701 .610258-2.304284$ \\
\hline $\mathrm{H}$ & $5.952805-2.695904-0.998345$ & $\mathrm{H}$ & $-3.8075622 .541496-2.858961$ \\
\hline C & $-0.157445-0.8490381 .832876$ & C & $-2.9572301 .193355-1.436693$ \\
\hline C & $0.855133-0.9205222 .800851$ & $\mathrm{H}$ & $-2.0736561 .806484-1.260719$ \\
\hline $\mathrm{H}$ & $1.766279-0.3303732 .677222$ & C & $-3.1190990 .002316-0.683200$ \\
\hline C & $0.716993-1.7431333 .914330$ & C & $0.553006-1.796855-2.182596$ \\
\hline $\mathrm{H}$ & $1.517502-1.7857384 .657347$ & C & $0.802819-0.433946-2.737235$ \\
\hline C & $-0.434597-2.5184574 .095524$ & C & $1.926851-0.143501-3.656784$ \\
\hline $\mathrm{H}$ & $-0.531442-3.1643684 .970228$ & $\mathrm{H}$ & $2.305570-1.094896-4.051782$ \\
\hline C & $-1.457119-2.4539573 .159823$ & $\mathrm{H}$ & $1.6245740 .504831-4.497771$ \\
\hline $\mathrm{H}$ & $-2.360679-3.0481363 .313559$ & $\mathrm{H}$ & $2.7572990 .367193-3.128908$ \\
\hline C & $-1.326520-1.6173732 .041450$ & & \\
\hline C & $-2.379286-1.3986701 .052831$ & \multicolumn{2}{|c|}{$\Lambda$-D-TS1 } \\
\hline C & 881.017 & & \\
\hline
\end{tabular}


02

Ir $\quad-0.025680-0.189870-0.016785$

O $\quad 0.4854121 .5517771 .194967$

O $\quad 1.4501043 .5674431 .129926$

N $\quad 0.7044201 .253806-1.456153$

H $\quad 0.4478511 .240470-2.444845$

N $\quad-2.0684290 .120718-0.449262$

N $\quad 1.908457-0.5475900 .419577$

C $\quad-0.986863-3.572297-3.298221$

H $\quad-1.230236-4.299824-4.074805$

C $-3.855310-0.516805-1.929641$

H $\quad-4.190838-1.154580-2.745721$

C $\quad 3.426520-0.8355632 .223149$

H $\quad 3.619057-1.1871053 .236250$

C $\quad 0.253280-3.615713-2.650001$

H $\quad 0.978383-4.387157-2.922106$

C $\quad-2.4676431 .7796221 .281995$

H $\quad-1.4491421 .6466281 .649102$

C $\quad-5.0868842 .1484200 .343683$

H $\quad-6.1055602 .276561-0.030271$

C $\quad 0.985945-2.2428743 .533187$

H $\quad 1.931659-2.3942024 .059875$
C $5.0614321 .055343-0.579093$

H $\quad 6.0308441 .314454-0.148514$

C $3.5148531 .071039-2.444559$

H $\quad 3.3265391 .288701-3.497919$

C $4.7322841 .451934-1.864479$

H $\quad 5.4507572 .024173-2.454325$

C $\quad-1.912155-2.604872-2.934019$

H $\quad-2.883364-2.586065-3.433367$

C $\quad-0.340519-1.685198-1.283519$

C $\quad 4.1373050 .3049750 .203896$

C $\quad 2.8723150 .014396-0.340458$

C $\quad-2.530949-0.660622-1.435138$

C $\quad-4.2436871 .205557-0.295229$

C $-1.598216-1.672338-1.932426$

C $2.5369840 .362756-1.707063$

C $\quad-0.271620-1.3410061 .614970$

C $\quad-4.6910780 .410187-1.381653$

H $\quad-5.7088840 .538416-1.757931$

C $\quad-2.9093951 .0258290 .165885$

C $2.135752-0.9934551 .664149$

C $\quad 0.9342972 .6022720 .601818$

C $\quad 0.949300-1.5486682 .316060$ 

C $\quad-1.395376-2.5709483 .387581$
$\Lambda$-L-TS1
H $\quad-2.318208-2.9782703 .809025$
02
C $0.569023-2.691559-1.659810$
Ir $\quad-0.0385320 .202694-0.008858$
H $\quad 1.539122-2.754935-1.158997$
O $\quad-0.493753-1.7073450 .937623$
C $\quad 0.7070722 .612439-0.934435$
O $\quad-1.213657-3.7956910 .591549$
C $\quad-1.436354-1.8741092 .182155$
N $\quad-0.662722-1.054498-1.640074$
H $\quad-2.394290-1.7382941 .673129$
H $\quad-0.374505-0.904889-2.609269$
C $\quad-0.187246-2.7566084 .068909$
N $\quad 2.0256010 .038405-0.393239$
H $\quad-0.165830-3.3029755 .013844$
N $\quad-2.0039350 .4187820 .407231$
C $\quad-3.3150822 .6814651 .884301$
C $\quad 0.8606423 .992433-2.825157$
H $\quad-2.9547083 .2515542 .742928$
H $\quad 1.0900374 .814424-3.505964$
C $4.393360-0.1587681 .521562$
C $\quad 3.8256750 .908649-1.731687$
H $\quad 5.3669800 .0375151 .976239$
H $\quad 4.1567581 .644591-2.462429$
C $\quad-4.6321432 .8787901 .414588$
C $\quad-3.5598450 .3340142 .201058$
H $\quad-5.2851363 .6026721 .905994$
H $\quad-3.7888300 .5236433 .249179$
H $\quad 1.918789-0.359890-2.246202$
C $\quad-0.3936443 .912231-2.208146$
C $1.5930483 .610131-1.659609$
H $\quad-1.1430404 .683373-2.405403$
H $\quad 1.4034523 .604513-2.744779$
C $\quad 2.445874-1.7816311 .162555$
H $\quad 2.6541483 .394058-1.479349$
H $1.410643-1.7356841 .503158$
H $\quad 1.3959474 .614690-1.265186$
C $5.104160-1.9423370 .274791$
H $\quad-0.3486922 .944116-1.039136$
H $\quad 6.137445-1.989111-0.077479$ 

C $\quad-1.2474561 .7253373 .743021$
C $\quad-0.854066-2.7015810 .202908$
H $\quad-2.2133671 .7610154 .253315$
C $\quad-1.1409571 .1946212 .450216$
C $\quad-4.971922-1.334749-0.850656$
C $\quad 1.1149302 .1907003 .716656$
H $\quad-5.906366-1.755839-0.473909$
H 2.0031732 .5891944 .213942
C $\quad-3.419400-0.953639-2.672888$
C $\quad-0.6927212 .865220-1.343125$
H $\quad-3.199437-1.024731-3.740365$
H $\quad-1.6746422 .828947-0.863247$
C $\quad-4.582249-1.547240-2.163886$
C $\quad-0.746833-2.472034-1.325880$
H $\quad-5.220497-2.134270-2.826927$
H $\quad-1.643639-2.920911-1.785854$
C $1.8174413 .024566-2.554496$
C $0.489344-3.203406-1.856965$
H $\quad 2.7986303 .103316-3.027978$
H $\quad 0.569439-3.116555-2.952349$
C $\quad 0.2491661 .856737-1.066658$
H $\quad 0.409033-4.266116-1.591875$
C $\quad-4.147307-0.5826400 .033184$
H $\quad 1.407052-2.799317-1.403541$
C $\quad-2.915477-0.098321-0.445514$
C $\quad 1.2255451 .6548442 .435406$
C $2.4822050 .943541-1.270014$
H 2.2020541 .6333841 .944385
C $4.240577-0.966327-0.281919$
C $\quad-0.1184712 .2277014 .376217$
C $\quad 1.5208641 .968615-1.677714$
H $\quad-0.1950632 .6524935 .378986$
C $-2.544616-0.226213-1.838573$
C $\quad 3.312229-2.7138101 .686363$
C $\quad 0.1064511 .1415911 .767328$
H $\quad 2.950489-3.3942882 .459723$
C $4.684285-0.039479-1.260168$
C $\quad-4.455670-0.3242641 .395780$
H $\quad 5.716924-0.085990-1.614380$
H $\quad-5.408307-0.6720011 .801941$
C $2.887967-0.8913690 .152035$
C $\quad 4.649092-2.8065901 .240775$
C $\quad-2.2787060 .6653141 .696614$
H $\quad 5.316893-3.5578631 .666890$ 


\section{$\Lambda$-L-TS2}

01

Ir $\quad 0.0514930 .0740160 .199799$

O $\quad 0.094764-1.7630051 .331710$

O $\quad 0.112487-3.9952621 .247285$

N $\quad-0.774580-1.525743-1.229962$

H $\quad-0.485590-1.345209-2.190933$

N $2.0610540 .187187-0.463470$

N $\quad-1.8560990 .0528760 .762357$

C $\quad 0.0578243 .847831-2.799051$

H $\quad 0.0908184 .673576-3.511793$

C $3.4748351 .141143-2.160316$

H $\quad 3.5710021 .828972-2.998813$

C $\quad-3.4894690 .6122942 .382733$

H $\quad-3.7699491 .1430493 .292162$

C $\quad-1.0504553 .679032-1.959568$

H $\quad-1.8773814 .393342-2.005004$

C $2.999206-1.3543001 .165009$

H $\quad 2.038342-1.4055861 .678123$
C $\quad-1.1293332 .2173753 .634750$

H $\quad-2.0837822 .3316484 .154677$

C $\quad-4.890574-1.809698-0.130197$

H $\quad-5.912548-1.9542210 .225128$

C $\quad-3.106607-2.412884-1.663391$

H $\quad-2.747354-3.010803-2.503796$

C $\quad-4.396889-2.570181-1.196545$

H $\quad-5.044449-3.315274-1.662824$

C $\quad 1.1287312 .970849-2.693518$

H $2.0044813 .129276-3.326301$

C $\quad-0.0480401 .686709-0.959639$

C $\quad-4.039935-0.8941530 .556355$

C $\quad-2.720976-0.7189690 .095114$

C $2.2351961 .049458-1.473908$

C $4.394258-0.486355-0.644970$

C $\quad 1.0905581 .917888-1.767235$

C $\quad-2.240592-1.336787-1.167618$

C $\quad 0.2001231 .2691811 .791806$

C $4.5291870 .370518-1.767178$

H $5.4858530 .420381-2.292565$ 
C $\quad-2.1696240 .7087521 .896534$

H $\quad 6.150494-2.6532991 .327993$

C $\quad-0.013063-2.8990700 .738804$

H $\quad-2.368259-0.505303-2.006966$

C $\quad-1.0380451 .4301182 .478310$

O $\quad-2.0374660 .193740-3.278173$

C $\quad 1.2214302 .7096043 .462766$

O $\quad-2.7177221 .331948-3.332207$

H 2.1106393 .2124573 .851532

H $\quad-2.1188891 .995679-2.927054$

C $\quad-1.1049852 .612299-1.062988$

H $\quad-1.9819542 .504010-0.418466$

$\Lambda-\left[\operatorname{lr}(p q)_{2}(\right.$ ala-2H) $]$

C $\quad-0.271682-2.846849-0.789052$

01

H $\quad-1.025344-3.623100-0.994306$

Ir $\quad 0.0075250 .089973-0.016596$

C $\quad 1.006335-3.194920-1.541820$

O $\quad-0.199854-1.9762600 .712923$

H $\quad 0.821090-3.294705-2.623295$

O $\quad-0.509591-4.0989780 .046186$

H $\quad 1.390866-4.150425-1.162633$

N $\quad-2.0128830 .1075640 .534784$

H $\quad 1.781098-2.428834-1.388388$

N $2.0564560 .204310-0.449173$

C $\quad 1.3194051 .9295902 .313078$

N $\quad-0.121422-1.174528-1.798527$

H 2.2851441 .8298051 .811505

C $\quad 0.1562000 .8924301 .804066$

C $\quad-0.0008302 .8581264 .127284$

C $\quad 1.2861621 .4522462 .419745$

H $\quad-0.0673323 .4713305 .027996$

H $\quad 2.2071081 .5863931 .846939$

C $4.069919-2.0907771 .616730$

C $\quad 1.2561961 .8358593 .756968$

H $\quad 3.944249-2.7127012 .505006$

H $\quad 2.1487732 .2694014 .215511$

C $-4.405243-0.1796041 .724841$

C $\quad-1.0397681 .1338253 .935459$

H $\quad-5.421113-0.2804712 .113634$ 

H $\quad-1.9464301 .0218914 .534069$
C $\quad 0.3895324 .216594-2.447910$
C $\quad-1.0144950 .7560442 .584379$
H $\quad 0.5065245 .126442-3.039703$
C $\quad-2.1810540 .2614731 .854113$
C $\quad 1.4513773 .336688-2.303065$
C $\quad-3.430433-0.0256992 .469077$
H $2.4033863 .568228-2.785981$
H $\quad-3.5252770 .0683343 .549445$
C $\quad 1.2970872 .171134-1.534554$
C $\quad-4.482191-0.4472471 .711911$
C $\quad 2.3780621 .227784-1.252414$
H $\quad-5.437152-0.7055502 .175280$
C $\quad 3.6990951 .382645-1.752421$
C $\quad-4.349014-0.5201040 .299737$
H $\quad 3.9203682 .209246-2.425610$
C $\quad-5.431328-0.866727-0.547158$
C $4.6728820 .500954-1.387962$
H $\quad-6.386829-1.134469-0.090138$
H $\quad 5.6908540 .602585-1.771962$
C $\quad-5.287047-0.847566-1.913274$
C $4.370931-0.547457-0.480767$
H $\quad-6.124731-1.110962-2.561619$
C $5.358816-1.455019-0.023969$
C $\quad-4.053014-0.454805-2.477237$
H $\quad 6.378061-1.352115-0.404127$
H $\quad-3.951753-0.387818-3.562559$
C $5.040154-2.4349370 .884414$
C $\quad-2.978752-0.138068-1.679256$
H $\quad 5.803677-3.1317401 .235540$
H $\quad-2.0440590 .204981-2.114815$
C $\quad 3.717950-2.5316251 .370675$
C $-3.088151-0.195005-0.267241$
H $\quad 3.464207-3.3005482 .102861$
C $\quad 0.0661451 .857079-0.910751$
C $\quad 2.733033-1.6723340 .940597$
C $\quad-0.9864662 .779085-1.059481$
H $\quad 1.714775-1.7688391 .316564$
H $\quad-1.9454272 .585798-0.572343$
C $3.033257-0.664854-0.009760$
C $-0.8276723 .935576-1.814103$
C $-0.338334-2.909794-0.148466$
H $\quad-1.6605424 .636996-1.911107$
C $-0.253626-2.431529-1.613132$ 
C $\quad-0.343132-3.458388-2.686100$

H $\quad-0.239317-3.024331-3.690283$

H $\quad 0.425328-4.226896-2.521295$

H $\quad-1.309662-3.975796-2.596554$

C $\quad 0.0962881 .6720934 .522736$

H $\quad 0.0814401 .9710645 .572680$

H $\quad-0.044365-0.881862-2.774531$

\section{$\Lambda$-[Ir(pq)(L-pqa)]}

01

Ir $\quad 0.0647130 .059641-0.061265$

O $\quad 0.037814-1.475589-1.595545$

O $\quad-0.064067-3.674847-1.984571$

N $\quad 0.886824-1.8275890 .956265$

H $\quad 0.660955-1.8730001 .949876$

N $\quad-1.9790040 .0783140 .513102$

N $1.9848210 .205347-0.531352$

C $\quad 0.0948142 .5302764 .068529$

H $\quad 0.0708593 .0385875 .034335$

C $\quad-3.4881290 .6970382 .281535$

H $\quad-3.6222961 .1589493 .258022$
C $3.7390421 .334858-1.655073$

H $4.0587872 .141041-2.314736$

C $\quad 1.2746332 .4908123 .317334$

H 2.1760622 .9757403 .701008

C $\quad-2.851907-0.927359-1.521830$

H $\quad-1.849255-0.946055-1.950541$

C $\quad-5.437711-0.924833-0.430890$

H $\quad-6.439013-0.9106580 .006135$

C $\quad 1.3846673 .130882-2.636956$

H $\quad 2.3652163 .399666-3.037931$

C $\quad 5.106252-1.5266170 .353321$

H $\quad 6.181115-1.4544460 .174165$

C $\quad 3.203176-2.6161661 .394903$

H $\quad 2.817239-3.4176042 .029300$

C $\quad 4.595902-2.5073291 .173749$

H $\quad 5.267220-3.2216281 .653391$

C $\quad-1.0497231 .9236783 .571348$

H $\quad-1.9691111 .9657224 .159348$

C $\quad 0.1717911 .2038421 .564637$

C $4.237714-0.593267-0.262563$

C $2.852701-0.699433-0.012187$

C $\quad-2.1915150 .6585441 .701188$ 

C $\quad-4.360926-0.3982920 .325056$
H $\quad 0.985180-3.8562770 .319148$
C $\quad-1.0179701 .2709242 .328823$
C $\quad-1.029032-3.3806990 .906133$
C $\quad 2.326381-1.7317430 .808152$
H $\quad-0.889610-3.6804551 .957392$
C $\quad-0.0284441 .663690-1.244978$
H $\quad-1.459721-4.2272460 .356406$
C $\quad-4.5500440 .1629321 .614107$
H $\quad-1.744550-2.5463160 .872053$
H $\quad-5.5507380 .1774542 .052534$
C $\quad-1.1317552 .440535-1.625444$
C $-3.044939-0.411331-0.215864$
H $\quad-2.1244192 .192988-1.241846$
C $\quad 2.3581301 .195782-1.338492$
C $\quad 0.2705083 .875102-2.997367$
C $0.085129-2.714539-1.255661$
H $\quad 0.3722174 .724868-3.674947$
C $\quad 1.2442472 .037934-1.770208$
C $-3.920080-1.426997-2.231213$
C $\quad-0.9845213 .524347-2.486480$
H $\quad-3.746949-1.827707-3.231835$
H $\quad-1.8630554 .110896-2.768136$
C $4.6542010 .467965-1.119551$
C $\quad 1.3114521 .8399912 .088689$
H $\quad 5.7170870 .582124-1.344099$
H $\quad 2.2492521 .8279201 .526897$
C $\quad-5.223304-1.435893-1.687812$
C $\quad 0.294383-3.0002490 .257316$
H $\quad-6.054584-1.842247-2.267182$ 


\section{S9. References}

(1) Li, A.-H.; Beard, D. J.; Coate, H.; Honda, A.; Kadalbajoo, M.; Kleinberg, A.; Laufer, R.; Mulvihill, K. M.; Nigro, A.; Rastogi, P.; Sherman, D.; Siu, K. W.; Steinig, A. G.; Wang, T.; Werner, D.; Crew, A. P.; Mulvihill, M. J. One-Pot Friedlander Quinoline Synthesis: Scope and Limitations. Synthesis-Stuttgart 2010, 1678-1686.

(2) Li, L.-P.; Yao, S.-Y.; Ou, Y.-L.; Wei, L.-Q.; Ye, B.-H. Diastereoselective Synthesis and Photophysical Properties of BisCyclometalated Ir(III) Stereoisomers with Dual Stereocenters. Organometallics 2017, 36, 3257-3265.

(3) Sheldrick, G. M. A Short History of SHELX. Acta Crystallogr. Sect. A: Found. Crystallogr. 2008, 64, 112-122.

(4) Dolomanov, O. V.; Bourhis, L. J.; Gildea, R. J.; Howard, J. A. K.; Puschmann, H. OLEX2: A Complete Structure Solution, Refinement and Analysis Program. J. Appl. Crystallogr. 2009, 42, 339-341.

(5) Frisch, M. J.; Trucks, G. W.; Schlegel, H. B.; Scuseria, G. E.; Robb, M. A.; Cheeseman, J. R.; Scalmani, G.; Barone, V.; Mennucci, B.; Petersson, G. A.; Nakatsuji, H.; Caricato, M.; Li, X.; Hratchian, H. P.; Izmaylov, A. F.; Bloino, J.; Zheng, G.; Sonnenberg, J. L.; Hada, M.; Ehara, M.; Toyota, K.; Fukuda, R.; Hasegawa, J.; Ishida, M.; Nakajima, T.; Honda, Y.; Kitao, O.; Nakai, H.; Vreven, T.; Montgomery, J. A.; Peralta, Jr., J. E.; Ogliaro, F.; Bearpark, M.; Heyd, J. J.; Brothers, E.; Kudin, K. N.; Staroverov, V. N.; Keith, T.; Kobayashi, R.; Normand, J.; Raghavachari, K.; Rendell, A.; Burant, J. C.; Iyengar, S. S.; Tomasi, J.; Cossi, M.; Rega, N.; Millam, J. M.; Klene, M.; Knox, J. E.; Cross, J. B.; Bakken, V.; Adamo, C.; Jaramillo, J.; Gomperts, R.; Stratmann, R. E.; Yazyev, O.; Austin, A. J.; Cammi, R.; Pomelli, C.; Ochterski, J. W.; Martin, R. L.; Morokuma, K.; Zakrzewski, V. G.; Voth, G. A.; Salvador, P.; Dannenberg, J. J.; Dapprich, S.; Daniels, A. D.; Farkas, O.; Foresman, J. B.; Ortiz, J. V.; Cioslowski, J.; Fox, D. J. Gaussian 09, rev. B.1; Gaussian, Inc.: Wallingford CT, 2013.

(6) Hohenberg, P.; Kohn, W. Inhomogeneous Electron Gas. Phys. Rev. 1964, 136, B864-B871.

(7) Chai, J. D.; Head-Gordon, M. Long-range corrected hybrid density functionals with damped atom-atom dispersion corrections. Phys Chem Chem Phys 2008, 10, 6615-6620.

(8) Weigend, F. Accurate Coulomb-fitting basis sets for H to Rn. Phys Chem Chem Phys 2006, 8, 1057-1065.

(9) Weigend, F.; Ahlrichs, R. Balanced basis sets of split valence, triple zeta valence and quadruple zeta valence quality for $\mathrm{H}$ to Rn: Design and assessment of accuracy. Phys Chem Chem Phys 2005, 7, 3297-3305.

(10) Marenich, A. V.; Cramer, C. J.; Truhlar, D. G. Performance of SM6, SM8, and SMD on the SAMPL1 Test Set for the Prediction of Small-Molecule Solvation Free Energies. J. Phys. Chem. B 2009, 113, 4538-4543.

(11) http://www.cylview.org/. 